\title{
ON THE APPROXIMATION-SOLVABILITY OF EQUATIONS INVOLVING $A$-PROPER AND PSEUDO- $A$-PROPER MAPPINGS ${ }^{1}$
}

\author{
BY W. V. PETRYSHYN
}

Table of Contents

\section{Introduction}

1. $A$-proper mappings and fixed point theorems for $P_{\gamma}$-compact mappings

1.1 Basic facts about $A$-proper mappings and approximation-solvability

1.2 Fixed point theory for $\boldsymbol{P}_{\gamma}$-compact mappings

2. Equations involving linear $A$-proper mappings

2.1 Approximation-solvability and bounded $A$-proper mappings

2.2 Equations involving unbounded linear mappings

2.3 Generalized Fredholm alternative

2.4 Dirichlet boundary value problem for elliptic equations

2.5 Stability of projective methods and $A$-properness

3. Equations involving nonlinear $A$-proper mappings

3.1 Approximation-solvability and nonlinear $A$-proper mappings

3.2 Equations involving odd $A$-proper mappings

3.3 Alternative for asymptotically linear mappings with applications to PDE

4. Generalized degree theory of $\boldsymbol{A}$-proper mappings and applications

4.1 Definition and properties of the generalized degree

4.2 Applications to some fixed point and mapping theorems

4.3 Fréchet differentiable and analytic type $A$-proper mappings

5. Existence theorems and pseudo- $A$-proper type mappings

5.1 Equations involving pseudo- $A$-proper mappings

5.2 Application to fixed point and surjectivity theorems for $G$-operators, $P_{1}$-compact, weakly closed, and monotone type operators

5.3 Equations involving uniform limits of $A$-proper type mappings

5.4 Surjectivity theorems for odd limits of $A$-proper mappings

\section{References}

An expanded version of an address delivered at the 78th Summer Meeting of the Society on August 24, 1973, by invitation of the Committee to Select Hour Speakers for Annual and Summer Meetings; received July 2, 1974.

AMS (MOS) subject classifications (1970). Primary 47A15, 47H10; Secondary $39 \mathrm{~A} 40$.

Key words and phrases. A-proper, pseudo- $A$-proper, $P$-compact, approximationsolvability, semicontractive, monotone, type (S), measure of noncompactness, condensing, continuum, Fredholm alternative, fixed point, surjective, generalized degree, Dirichlet boundary value problems.

${ }^{1}$ This work was supported in part by NSF Grant GP-20228. 


\section{Introduction}

Let $X$ and $Y$ be Banach spaces, $D$ a given subset of $X, T: D \subseteq X \rightarrow Y$ a possibly nonlinear mapping and $\left\{X_{n}, P_{n} ; Y_{n}, Q_{n}\right\}$ a suitable approximation scheme for the equation

$$
T(x)=f \quad(x \in D, f \in Y) .
$$

One of the basic problems of functional analysis is to solve equation (I). In this paper we distinguish in principle between two notions of solvability of the above problem, namely:

(A) Solvability of equation (I) in which the existence of a solution of equation (I) is somehow established.

(B) Approximation-solvability of equation (I) in which a solution of equation (I) is obtained as a limit (or at least a limit point) of solutions $x_{n}$ of simpler finite dimensional equations

$$
T_{n}\left(x_{n}\right)=Q_{n} f \quad\left(x_{n} \in D_{n}, Q_{n} f \in Y_{n}\right) .
$$

Needless to say, although the concepts (A) and (B) are distinct in their purpose, they are not independent. In fact, as will be seen below, in some cases the knowledge of (A) is essential for (B) to take place.

In the classical functional analysis, the above problem has been handled satisfactorily if a given equation is reducible to one in which $T$ is either of the form $T=I-S$ with $S: D \subseteq X \rightarrow X$ contractive or $T=I-C$ with $C: D \subseteq X \rightarrow X$ compact. The contraction mapping principle, Schauder fixed point theorem, Leray-Schauder degree theory for $T=I-C$, Galerkin method for $T=I-C$ and their consequences provided the basis for the treatment of equation (I) for these special classes of mappings.

The main thrust of the recent development of nonlinear functional analysis has been in the direction of breaking out of the classical framework into a much wider field of noncompact operators such as: operators of monotone and accretive type; operators of ultimately compact and setcontractive type; operators of $A$-proper type; and others. There are extensive surveys and treatments of the theories and applications of the first two classes of mappings. See, for example [5], [19], [57], [81], [98], for the discussion of operators of monotone and accretive type and their applications and [95], [122], [136] for the discussion of operators of ultimately compact, condensing, and set-contractive type.

The purpose of this paper is to survey the basic results from the theory of $A$-proper mappings developed recently in the context of constructive functional analysis which came about as the answer to the following problem. For what type of a linear or nonlinear mapping $T$ is it possible to construct a solution of equation (I) as a strong limit of solutions $x_{n}$ of 
equation (II)? In a series of papers the author studied this problem and the notion which evolved from these investigations is that of an $A$-proper mapping. It turned out that the $A$-properness of $T$ is not only intimately connected with the approximation-solvability of equation (I) but, in view of the fact that the class of $A$-proper mappings is quite extensive, the theory of $A$-proper mappings extends and unifies earlier results concerning Galerkin type methods for linear and nonlinear equations with the more recent results in the theory of strongly monotone and accretive operators, operators of type (S), $P_{\gamma}$-compact, ball-condensing and other mappings. In view of this, the class of $A$-proper mappings and its precursor, the class of $P_{\gamma}$-compact mappings, became a subject of extensive study by a number of authors, including Browder, Browder and Petryshyn, Deimling, Edmunds-Webb, Fitzpatrick, Fučik, Goncharov, Grigorieff, Hamilton, Nussbaum, O'Neil-Thomas, Petry, Petryshyn, Petryshyn and Tucker, Pokhodjayev, Potter, Stuart, Tucker, Webb, Wolf, Wong and others. As a result, the theory of $A$-proper mappings has been developed and extended in various directions.

In this paper we survey some basic results from this theory and indicate their relations to earlier approximation results in linear and nonlinear theory of the Galerkin type methods and to the more recent results of the nonlinear theory involving operators of monotone and accretive type, $\boldsymbol{P}_{\boldsymbol{\gamma}}$-compact, condensing, type (S) and other mappings.

In the course of survey the author noted that the notion of an $A$-proper mapping is also closely related to the stability of the projectional method (II) in the sense of Mikhlin and that it enters rather naturally in the solvability of elliptic partial differential equations. These and other new observations are included in this survey and in some cases their proofs are sketched.

We call the reader's attention to the fact that for obvious reasons our survey makes no attempt to present the theory of $A$-proper mappings in its most general form. For the sake of simplicity we present the outline for real Banach spaces $X$ and $Y$ equipped with a projectionally complete scheme $\Gamma$ although, as will be indicated below, a number of approximationsolvability results are valid for complex Banach spaces and for more general approximation schemes.

Finally, we remark that the topics which we shall treat in this paper are stated in the Table of Contents.

\section{1. $A$-proper mappings and fixed point theorems for $\boldsymbol{P}_{\boldsymbol{\gamma}}$-compact mappings}

In $\$ 1.1$ we fix the terminology and notation, introduce the definitions of some basic concepts used in this paper, give a historical background and some examples of $A$-proper mappings and mention a couple of properties 
that are relevant to this section. In $\$ 1.2$ we outline the fixed point theory for $P_{\gamma}$-compact mappings and show that it includes the classical fixed point theorems for compact mappings and the recent fixed point theorems for ball-condensing and strictly semicontractive mappings.

1.1. Basic facts about A-proper mappings and approximate-solvability. Let $X$ and $Y$ be real separable Banach spaces with $X^{*}$ and $Y^{*}$ denoting their respective duals. We use " $\rightarrow$ " and " $\rightarrow$ " to denote the strong and the weak or the weak* convergence respectively. Let $D$ be a given set in $X$ and $T: D \subseteq X \rightarrow Y$ a possibly nonlinear mapping. For the sake of simplicity we define the notion of the A-properness of $T$ and of the approximationsolvability of

$$
T x=f \quad(x \in D, f \in Y)
$$

in terms of a given projectionally complete scheme $\Gamma$ for $(X, Y)$.

Definition 1.1A. The scheme $\Gamma=\left\{X_{n}, P_{n} ; Y_{n}, Q_{n}\right\}$ is projectionally complete for $(X, Y)$ provided that $\left\{X_{n}\right\} \subset X$ and $\left\{Y_{n}\right\} \subset Y$ are sequences of monotonically increasing finite dimensional subspaces with $\operatorname{dim} X_{n}=$ $\operatorname{dim} Y_{n}$ for each $n$ and $P_{n}: X \rightarrow X_{n}$ and $Q_{n}: Y \rightarrow Y_{n}$ are linear projections such that $P_{n} x \rightarrow x$ and $Q_{n} y \rightarrow y$ for $x \in X$ and $y \in Y$.

In case $Y=X, Y_{n}=X_{n}$ and $Q_{n}=P_{n}$ we denote the scheme $\left\{X_{n}, P_{n}\right.$; $\left.X_{n}, P_{n}\right\}$ for $(X, X)$ by $\Gamma_{0}$.

It is obvious that if $\left\{\phi_{i}\right\} \subset X$ and $\left\{\psi_{i}\right\} \subset Y$ are Schauder bases, then there exists a natural projectionally complete scheme $\Gamma=\Gamma_{S}$. Namely, one chooses $X_{n}=\left[\phi_{i}, \cdots, \phi_{n}\right], Y_{n}=\left[\psi_{1}, \cdots, \psi_{n}\right]$,

$$
P_{n}(x)=\sum_{i=1}^{n}\left(\Phi_{i}, x\right) \phi_{i} \quad \text { and } \quad Q_{n}(y)=\sum_{i=1}^{n}\left(\Psi_{i}, y\right) \psi_{i},
$$

where $\left\{\Phi_{i}\right\} \subset X^{*}$ and $\left\{\Psi_{i}\right\} \subset Y^{*}$ are such that $\left(\Phi_{i}, \phi_{j}\right)=\left(\Psi_{i}, \psi_{j}\right)=\delta_{i j}$ with $(w, x)$ denoting the value of $w$ in $X^{*}$ at $x$ in $X$. To incorporate the Galerkin type methods into the framework of the $A$-proper mapping theory, it is only necessary to assume that $P_{n}$ and $Q_{n}$ are orthogonal projections if $X$ and $Y$ are Hilbert spaces.

In [107], [109], [110] the author investigated the type of mappings $T$ for which it is possible to construct a solution $x \in D$ of equation (1.1-1) as a strong limit of solutions $x_{n} \in D_{n}=D \cap X_{n}$ of approximate equations

$$
T_{n}(x)=Q_{n} f \quad\left(x \in D_{n}, T_{n}=\left.Q_{n} T\right|_{D_{n}}\right) .
$$

In [111] (see also [112]) the author introduced a wide class of mappings, those mappings satisfying condition $(\mathrm{H})$, which proved to be very suitable for the study of the constructive solvability of equation (1.1-1). In [113] and subsequently, mappings satisfying condition $(\mathrm{H})$ have been referred to as approximation-proper ( $A$-proper). 
Definition 1.1B. $T: D \subseteq X \rightarrow Y$ is said to be $A$-proper with respect to the projectionally complete scheme $\Gamma$ if $T_{n}: D_{n} \subseteq X_{n} \rightarrow Y_{n}$ is continuous for each $n$ and if $\left\{x_{n_{j}} \mid x_{n_{j}} \in D_{n_{j}}\right\}$ is any bounded sequence such that $T_{n_{j}}\left(x_{n_{j}}\right) \rightarrow g$ for some $g$ in $Y$, then there exist a subsequence $\left\{x_{n_{j(k)}}\right\}$ and $x \in D$ such that $x_{n_{j(k)}} \rightarrow x$ and $T x=g$.

For later use we recall the following definitions. If $T: D \subseteq X \rightarrow Y$, then $T$ is bounded if $T(Q)$ is bounded whenever $Q \subset D$ is bounded; $T$ is compact if $T$ is continuous on $D$ and $T(Q)$ is precompact in $Y$ whenever $Q \subset D$ is bounded; $T$ is weakly continuous on $D$ if $x_{n} \rightarrow x$ implies $T x_{n} \rightarrow T x$ in $Y$ for $x_{n}, x \in D ; T$ is completely continuous if $x_{n} \rightarrow x$ in $X$ implies $T x_{n} \rightarrow T x$ for $x_{n}, x \in D ; T$ is fa-continuous if $T_{n}: D_{n} \subset X_{n} \rightarrow Y_{n}$ is continuous for each $n ; T$ is contractive (resp. nonexpansive) if $\|T x-T y\| \leqq l\|x-y\|$ for $x$, $y \in D$ with $0<l<1$ (resp. $l=1$ ); $T$ is proper if $T^{-1}(Q)$ is compact whenever $Q$ is compact; the space $X$ provided with a scheme $\Gamma_{0}$ with $\left\|P_{n}\right\|=1$ for each $n$ is called a $\Pi_{1}$-space; $B\left(x_{0}, r\right)$ and $\bar{B}\left(x_{0}, r\right)$ are used to denote the open and the closed ball in $X$ respectively with center at $x_{0}$ and radius $r$.

The following result of the author [117] provides the motivation for the terminology " $A$-proper" and is useful in other respects.

Proposition 1.1C. If $D \subset X$ is open and $T: \bar{D} \rightarrow Y$ is continuous and $A$-proper, then the restriction of $T$ to any closed bounded subset of $\bar{D}$ is proper.

An example has been given by Fitzpatrick [39] which shows that a continuous proper mapping need not be $A$-proper. The following example from [116] which illustrates this fact will also prove to be important in our discussion of the relationship between solvability, approximationsolvability and $A$-properness.

EXAMPLE 1.1a. Let $H$ be a real Hilbert space with a complete orthonormal basis $\left\{\cdots \phi_{-3}, \phi_{-2}, \phi_{-1}, \phi_{0}, \phi_{1}, \phi_{2}, \cdots\right\}, \tilde{X}_{n}=\left[\phi_{-n}, \cdots, \phi_{n}\right], \tilde{P}_{n}$ the orthogonal projection of $H$ onto $\tilde{X}_{n}$ and $T$ a bounded linear map of $H$ into $H$ given by $T_{\phi_{i}}=\phi_{i-1}$ for $i=0, \pm 1, \pm 2, \cdots$. It follows easily that $T$ is a one-to-one mapping of $H$ onto $H$ and so is also proper. But it is not hard to verify that $T$ is not $A$-proper with respect to $\tilde{\Gamma}_{0}=\left\{\tilde{X}_{n}, \widetilde{P}_{n} ; \tilde{X}_{n}, \widetilde{P}_{n}\right\}$.

Before we give some examples of $A$-proper mappings which would indicate the generality of this class, we first state the following useful property obtained by the author in [112].

Proposition 1.1D. If $T: D \subseteq X \rightarrow Y$ is A-proper with respect to $\Gamma$ and $C: D \rightarrow Y$ is compact, then $T+C$ is A-proper with respect to $\Gamma$.

However, the following example from [116] shows that a sum of two $A$-proper maps need not be $A$-proper.

EXAMPLE 1.1b. Let $H$ be a Hilbert space with a complete orthonormal basis $\left\{\phi_{i}\right\}, X_{n}=\left[\phi_{1}, \cdots, \phi_{n}\right], P_{n}$ the orthogonal projection of $H$ onto 
$X_{n}$ and $T$ a bounded linear mapping of $H$ onto $H$ given by

$$
T \phi_{1}=0, \quad T \phi_{2}=0, \quad T \phi_{3}=\phi_{2}, \cdots, \quad T \phi_{i}=\phi_{i-1} \quad \text { for } i \geqq 3 .
$$

It is easy to see that $T$ is $A$-proper with respect to $\Gamma_{0}=\left\{X_{n}, P_{n} ; X_{n}, P_{n}\right\}$. Indeed, since $T_{n} x=T x$ for $x \in X_{n}$ and since $\|T x\| \geqq\left\|x-P_{2} x\right\|$ it follows that $T$ is $A$-proper with respect to $\Gamma_{0}$. It is obvious that the identity $I$ on $H$ is $A$-proper with respect to $\Gamma_{0}$. But $I+T$ is not $A$-proper with respect to $\Gamma_{0}$. In fact, if $x_{n}=n^{-1 / 2}\left(\phi_{1}-\phi_{2}+\phi_{3}+\cdots+(-1)^{n} \phi_{n}\right) \in X_{n}$ then $\left\|x_{n}\right\|=1$ and $x_{n}+T_{n} x_{n} \rightarrow 0$ while, for $n>m,\left\|x_{n}-x_{m}\right\|^{2} \geqq n^{-1}(n-m)$. Therefore $I+T$ is not $A$-proper with respect to $\Gamma_{0}$.

The above fact has an important bearing, for example, on the homotopy theorem in the generalized degree theory for $A$-proper mappings. Examples will be given in Chapter 2 which show that an adjoint $T^{*}$ of an $A$-proper mapping $T: H \rightarrow H$ need not be $A$-proper. All this is not surprising since, as we shall see in Chapter 2, the $A$-properness of a mapping $T \in L(X, Y)$ characterizes the approximation-solvability (defined below) of equation (1.1-1). The following are some of the examples (others will be given later) of $A$-proper mappings.

EXAMPLE 1.1c. If $D \subset X$ is closed and $C: D \rightarrow X$ is compact, then $T=I-C$ is $A$-proper with respect to $\Gamma_{0}$.

EXAMPLE 1.1d. If $X$ is a $\Pi_{1}$-space, $D \subset X$ closed, $C: D \rightarrow X$ compact and $S: X \rightarrow X$ contractive, then $T=I-S-C: D \rightarrow X$ is $A$-proper.

It is unknown if $T=I-S-C$ is $A$-proper in a general $\Pi_{1}$-space $X$ if $S$ is defined and contractive only on $D$ even when $D=\bar{B}(0,1)$. See $\$ 1.2$ for further discussion of this case. It was noted above that the notion of $A$-properness evolved from the concept of a Projectionally-compact ( $P$ compact) mapping introduced by the author in [107] for the constructive approach to fixed point and eigenvalue problems for noncompact maps and to surjectivity theorems for monotone maps acting in Hilbert spaces (see [89], [10], [140], [108]). The definition of $P$-compactness used in [107] can be stated by

Definition 1.1E. A map $F: D \subseteq X \rightarrow X$ is $P$-compact if and only if $T_{\lambda}=F-\lambda I$ is $A$-proper with respect to $\Gamma_{0}$ for each $\lambda>0$.

EXAMPLE 1.1e. If $H$ is a Hilbert space and $F: H \rightarrow H$ is bounded and monotone decreasing (i.e. $(F x-F y, x-y) \leqq 0$ for $x, y \in H)$ and either continuous, demicontinuous, or weakly continuous, then $-F$ is $P$ compact (see [108]).

We add in passing that the study of monotone mappings was initiated independently by Vainberg, Kačurovskiř and Zarantonello (see [19]) and was then extensively studied by Minty, Browder, Rockafellar, Brezis and many others (see [19], [57], [81] for references). 
To introduce our next example, we first recall that if $Q$ is any bounded set in $D$, then the ball measure of noncompactness $\chi(Q)$ of $Q$ with respect to $X$ is defined to be

$\chi(Q)=\inf \{r>0 \mid Q \quad$ can be covered by a finite number of balls with radii $\leqq r$ and centers in $X$ \}.

It follows that $\chi(Q)=0$ if and only if $\bar{Q}$ is compact. The measure $\chi(Q)$ has a lot of useful properties (see [136]). We say that a continuous bounded mapping $F: D \subseteq X \rightarrow X$ is $k$-ball-contractive if $\chi(F(Q)) \leqq k \chi(Q)$ for every bounded $Q \subset D$ and some $k \geqq 0$. We see that $F$ is compact if and only if $F$ is 0-ball-contractive. Following Sadovsky [136] we say that $F: D \subseteq X \rightarrow X$ is ball-condensing if $\chi(F(Q))<\chi(Q)$ whenever $Q \subset D$ is bounded and $\chi(Q) \neq 0$. Of course, every $k$-ball-contractive map with $k<1$ is ball-condensing but the converse is not true (see [93]). Ball-condensing mappings have been recently a subject of extensive study (see [136], [122]). The following result is due to Webb [156] although in a somewhat different form it was already contained in [93].

EXAMPLE 1.1f. If $X$ is a $\Pi_{1}$-space and $F: D \subseteq X \rightarrow X$ is ball-condensing, then $T=I-F$ is $A$-proper.

EXAmple 1.1g. Let $X$ be reflexive with $\Gamma_{1}=\left\{X_{n}, P_{n} ; Y_{n}, Q_{n}\right\}$ projectionally complete for $\left(X, X^{*}\right)$ where $Q_{n}=P_{n}^{*}: X^{*} \rightarrow Y_{n}=R\left(Q_{n}\right)$. Let $T: X \rightarrow X^{*}$ be strongly monotone, i.e. $(T x-T y, x-y) \geqq\|x-y\|^{2}$ for $x$, $y \in X$ and some $c>0$, and either continuous, demicontinuous, or weakly continuous, then $T$ is $A$-proper with respect to $\Gamma_{1}$.

For the case when $X$ is a Hilbert space and $T$ is also bounded when it is demicontinuous the above fact was proved by the author [108], [109]. For bounded and demicontinuous $T: X \rightarrow X^{*}$, it has been proved in [24] while for unbounded demicontinuous $T: X \rightarrow X^{*}$, it follows from a more general result in [119].

Following Browder [19] we say that $T: X \rightarrow X^{*}$ satisfies condition (S) whenever $x_{n} \rightarrow x$ and $\left(T x_{n}-T x, x_{n}-x\right) \rightarrow 0$ imply that $x_{n} \rightarrow x$. The following fact was proved in [19].

EXAMPLE 1.1h. Let $X$ be as in Example 1.1g. If $T: X \rightarrow X^{*}$ is a bounded continuous map which satisfies condition (S), then $T$ is $A$-proper with respect to $\Gamma_{1}$.

We may add that Browder proved the above result for the case when the approximation scheme is given by an injective scheme but the same argument works for projective schemes. Moreover, if $T$ is bounded, it suffices to assume that $T$ is demicontinuous.

To complete our initial list of examples of $A$-proper mappings, we first recall that a continuous function $\mu: R^{+}=\{t \mid t \geqq 0\} \rightarrow R^{+}$is called a gauge function if $\mu(0)=0$ and $\mu$ is strictly increasing. If $J: X \rightarrow X^{*}$, then 
$J$ is said to be a duality mapping of $X$ with gauge function $\mu$ if

$$
(J x, x)=\|x\| \mu(\|x\|) \text { and }\|J x\|=\mu(\|x\|) \text { for } x \in X .
$$

If $\mu(t)=t$ we say that $J$ is a normalized duality map. If $X^{*}$ is strictly convex, then $J$ is uniquely determined by $\mu$ and if $X$ is also reflexive, then $J$ is demicontinuous. Moreover, if $X$ and $X^{*}$ have Property $(\mathrm{H})$ and, in particular, are locally uniformly convex, then $J$ is continuous (see [12] and [22]). Recall that $X$ has Property (H) if $X$ is strictly convex and such that if $x_{n} \rightarrow x$ and $\left\|x_{n}\right\| \rightarrow\|x\|$, then $x_{n} \rightarrow x$ in $X$. The following fact proved by the author in [117] plays an essential role in the generalized topological degree theory for mappings $T: D \subseteq X \rightarrow X^{*}$.

EXAmple 1.1i. If $X$ is reflexive and $X$ and $X^{*}$ have Property $(\mathrm{H})$, then the normalized duality mapping $J: X \rightarrow X^{*}$ is $A$-proper with respect to $\Gamma_{1}$.

The above examples (more complicated ones will be discussed later) indicate that the class of $A$-proper mappings is quite general and consequently any result one can obtain for $A$-proper mappings will include many results obtained for various classes usually by different and, as a rule, nonconstructive methods.

It was noted above that in trying to characterize the class of mappings $T$ for which a solution $x \in D$ of equation (1.1-1) can be obtained as a strong limit of solutions $x_{n} \in D_{n}$ of equation (1.1-2) the author was led to the notion of an $A$-proper mapping. To state the results concerning the approximation-solvability mentioned in the Introduction precisely and to indicate the intimate relationship of the $A$-properness of $T$ to the constructive solvability of equation (1.1-1) via the projection method (1.1-2) (or even more general approximation methods) we need the following.

DEFINITION 1.1F. (a) Equation (1.1-1) is uniquely approximationsolvable with respect to $\Gamma$ if there exists an $n_{0} \geqq 1$ such that equation (1.1-2) has a unique solution $x_{n} \in D_{n}$ for each $n \geqq n_{0}$ such that $x_{n} \rightarrow x$ and $x$ is the unique solution of equation (1.1-1).

(b) Equation (1.1-1) is strongly (resp. feebly) approximation-solvable with respect to $\Gamma$ is there exists $n_{0} \geqq 1$ such that equation (1.1-2) has a solution $x_{n} \in D_{n}$ for each $n \geqq n_{0}$ such that $x_{n} \rightarrow x \in D$ (resp. $x_{n_{j}} \rightarrow x$ for some subsequence $\left.\left\{x_{n_{j}}\right\}\right)$ and $T(x)=f$.

Chapters 2 and 3 and part of Chapter 4 are devoted to the survey of results concerning the relationship of the $A$-properness of $T$ to the unique or strong approximation-solvability of equation (1.1-1). Applications to various abstract and differential equations are considered.

ReMARK 1.1-1. It was noted in the Introduction that for the sake of simplicity we survey the results from the theory of $A$-proper mappings $T$ for the case when the $A$-properness of $T$ is defined in terms of projectionally complete schemes. However, many results in this theory are known 
to be also valid when the $A$-properness of $T$ is defined in terms of more general approximation schemes. The following definition of an admissible scheme illustrates the type of generality we have in mind, although other and still more general schemes have also been used (see [24], [115] for details).

Definition 1.1G. Let $\left\{E_{n}\right\}$ and $\left\{F_{n}\right\}$ be two sequences of oriented finite dimensional spaces with $\operatorname{dim} E_{n}=\operatorname{dim} F_{n}$ and let $\left\{V_{n}\right\}$ and $\left\{W_{n}\right\}$ be two sequences of continuous linear mappings with $V_{n}$ mapping $E_{n}$ into $X$ and $W_{n}$ mapping $Y$ onto $F_{n}$ such that $\left\{V_{n}\right\}$ and $\left\{W_{n}\right\}$ are uniformly bounded, $\operatorname{dist}\left(x, V_{n} E_{n}\right) \rightarrow 0$ for every $x \in X$, and for some $r>0$ the set $V_{n}^{-1}(B(0, r))=\left\{x \in E_{n} \mid V_{n} x \in B(0, r)\right\}$ is bounded for each $n$. We call $\Gamma_{3}=\left\{E_{n}, V_{n} ; F_{n}, W_{n}\right\}$ an admissible approximation scheme for mappings $T: X \rightarrow Y$.

Note that we do not require that $E_{n}$ and $F_{n}$ be subspaces of $X$ and $Y$, respectively, or that $V_{n}$ and $W_{n}$ be linear projections. Consequently, in addition to projective schemes considered in this paper, the schemes $\Gamma_{3}$ include injective schemes which always exist when $X$ and $Y$ are separable, finite difference schemes, and others. Moreover, a projective scheme could be admissible for mappings $T: X \rightarrow X^{*}$ without being projectionally complete for $\left(X, X^{*}\right)$ in the sense of Definition 1.1A.

Definition 1.1H. $T: X \rightarrow Y$ is said to be $A$-proper with respect to an admissible approximation scheme $\Gamma_{3}$ if $T_{n} \equiv W_{n} T V_{n}: E_{n} \rightarrow F_{n}$ is continuous and if for any sequence $\left\{x_{n_{j}} \mid x_{n_{j}} \in E_{n_{j}}\right\}$ such that $\left\{V_{n_{j}}\left(x_{n_{j}}\right)\right\}$ is bounded in $X$ and $\left\|T_{n_{j}}\left(x_{n_{j}}\right)-W_{n_{j}} f\right\| \rightarrow 0$ for some $f$ in $Y$, there exist $\left\{x_{n_{j(k)}}\right\}$ and $x$ in $X$ such that $V_{n_{j(k)}} x_{n_{j(k)}} \rightarrow x$ in $X$ and $T(x)=y$.

In a similar fashion one defines the approximation-solvability concepts. The case when $T$ is defined on a proper subset $D$ of $X$ can be handled similarly. It is known (see [24], [112], [115]) that many results obtained for $A$-proper mappings given by Definition 1.1B carry over to mappings given by Definition 1.1H. See also a series of papers by Grigorieff, Jeggle, Wolf and others, where the $A$-properness is studied in terms of the notion of a discrete convergence introduced by Stummel [145].

Reading this article the reader should always keep in mind the fact that we are usually given not only the operator $T: D \subseteq X \rightarrow Y$ but also an approximation scheme $\Gamma$ with respect to which $T$ may or may not be $A$-proper.

1.2. Fixed point theory for $P$-compact and generalized $P$-compact mappings. In this section we outline in chronological order some basic facts obtained in the theory of $P$-compact and generalized $P$-compact (or $P_{\gamma}$-compact) mappings whose study was initiated by the author in [107] and in [111], respectively, and continued by the writer (see [108], [106]) and a number of other authors (see [24], [30], [31], [34], [39], [77], [101], [114], [146]). 
The first result in this direction was established in [107], [108] by a simple use of a retraction argument and the Brouwer fixed point theorem.

THEOREM 1.2A. Suppose $T: \bar{B}(0, r) \subset X \rightarrow X$ is bounded and P-compact with respect to $\Gamma_{0}$, and that for some $\mu>0$ the following is true:

$$
\text { If } T x=\alpha x \text { and } x \in \partial B(0, r), \text { then } \alpha \leqq \mu .
$$

Then there exists $x_{0}$ in $\bar{B}(0, r)$ which satisfies the equation

$$
T x-\mu x=0
$$

if $\left(\pi_{\mu}^{<}\right)$holds on $\partial B(0, r)$ (i.e., if $T x=\alpha x$ and $x \in \partial B(0, r)$, then $\left.\alpha<\mu\right)$, then equation (1.2-1) is feebly approximation-solvable with respect to $\Gamma_{0}$, and strongly approximation-solvable if $x_{0}$ is unique.

If $\mu=1$, then the second part of Theorem $1.2 \mathrm{~A}$ implies the constructive existence of a fixed point of $T$ in $B(0, r)$ and thus includes the result of Krasnoselsky [69] concerning the convergence of the Galerkin method for the case when $T$ is compact (see [72] for earlier contributions).

REMARK $1.2-1$. Condition ( $\left.\pi_{1}^{\leqq}\right)$is implied by any one of the following:

(A1) $\|T x-x\|^{2} \geqq\|T x\|^{2}-\|x\|^{2}$ for $x \in \partial B(0, r)$ (used in [2]). $J=I$ ).

(A2) $(T x, J x) \leqq(x, J x)$ for $x \in \partial B(0, r)$ (used in [67] for $X=H$ and

(A3) $T x \in \bar{B}(0, r)$ for $x \in \partial B(0, r)$ (used in [135]).

Consequently, since every compact $T: \bar{B} \rightarrow X$ is $P$-compact, the fixed point theorems of Schauder [138], Rothe [135], Leray and Schauder [80], Altman [2] and Krasnoselsky [67] follow from Theorem 1.2A. Moreover, the theorem of Kaniel [62] for quasicompact $T$ follows also from Theorem 1.2A. We add that Theorem 1.2A was used in [106], [108] to deduce the constructive versions of the surjectivity theorems of Minty [89], Browder [10] and Shinbrot [140] for $T_{\lambda}=\lambda-A$ where $\lambda>0$ and $-A: H \rightarrow H$ is monotone and bounded and either continuous, demicontinuous, or weakly continuous.

It was shown in [106] that Theorem 1.2A remains valid if the boundedness assumption on $T$ is replaced by the weaker condition:

$(\Delta)$ There exists $c>0$ such that if $T_{n}(x)=\lambda x$ holds for $x \in X_{n} \cap \partial B(0, r)$ and any $n$ with $\lambda>0$, then $\lambda \leqq c$.

It follows from the strengthened version of Theorem 1.2A that we have

THEOREM 1.2B. Suppose $T: X \rightarrow X$ is $P$-compact and $\left\{c_{p}\right\} \equiv\left\{c\left(r_{p}\right)\right\}$ and $\left\{k_{p}\right\} \equiv\left\{k\left(r_{p}\right)\right\}$ are sequences with $k_{p} \rightarrow \infty$ as $r_{p} \rightarrow \infty$ such that:

$\left(\Delta_{f}\right)$ If $P_{n} T x-\lambda x=P_{n} f$ holds for $x \in \partial B\left(0, r_{p}\right), f \in B\left(0, r_{p}\right)$ and any $n$ with $\lambda>0$, then $\lambda \leqq c_{p}$.

$\left(\Pi_{p}\right)\|T x-\eta x\| \geqq k_{p}$ for $\eta \geqq \mu, x \in \partial B\left(0, r_{p}\right)$ and some $\mu>0$. 
Then the equation

$$
T x-\mu x=f \quad(f \in X)
$$

is feebly approximation-solvable for each $f \in X$. Equation (1.2-2) is strongly approximation-solvable if it is uniquely solvable.

The following corollaries of Theorem 1.2B illustrate its generality and practical usefulness.

Corollary 1.2C. If $T: X \rightarrow X$ is $P$-compact, bounded and $\|T x\| /\|x\| \rightarrow 0$ as $\|x\| \rightarrow \infty$, then for any given $\mu>0$ the conclusions of Theorem 1.2B hold.

Corollary 1.2D. If $T: X \rightarrow X$ is P-compact and $(T x, J x) \leqq(T(0), J x)$ for $x \in X$, then for any given $\mu>0$ the conclusions of Theorem 1.2B hold.

Following Granas [47] we say that $T: X \rightarrow X$ is quasibounded if there exist $M>0$ and $q_{0}>0$ such that $\|T x\| \leqq M\|x\|$ for $\|x\| \geqq q_{0}$. If $T$ is quasibounded, then its quasinorm $|T|$ is given by

$$
|T|=\inf _{a_{0} \leqq \alpha<\infty}\left\{\sup _{\|x\| \geqq q} \frac{\|T x\|}{\|x\|}\right\} .
$$

It was shown in [47] that if $T$ has an asymptotic derivative $T_{\infty}$ (see $\$ 3.3$ ), then $T$ is quasibounded and $|T| \leqq\left\|T_{\infty}\right\|$. Another corollary of Theorem $1.2 \mathrm{~B}$ is the following generalization of the result in [47].

COROLlaRY 1.2E. If $T: X \rightarrow X$ is $P$-compact and quasibounded, then for each $\mu>|T|$ the conclusions of Theorem 1.2B hold.

It was noted by the writer in [111] that if one is interested only in fixed point theorems for $T: D \subset X \rightarrow X$, then it suffices to assume that $T$ is $P_{1}$-compact. This observation led to the class of $P_{\gamma}$-compact mappings defined in [111] as follows.

Definition 1.2F. Given $\gamma \geqq 0 . T: D \subset X \rightarrow X$ is said to be a generalized $P$-compact or $\boldsymbol{P}_{\gamma}$-compact if for each $\lambda$ dominating $\gamma$ (i.e. $\lambda \geqq \gamma$ if $\gamma>0$ and $\lambda>\gamma$ if $\gamma=0$ ) the map $T_{\lambda}=T-\lambda I$ is $A$-proper with respect to $\Gamma_{0}$. Thus $T$ is $P$-compact if and only if $T$ is $P_{0}$-compact.

The study of $P_{\gamma}$-compact mappings, which include $P$-compact mappings when $\gamma=0$, was further continued in Petryshyn and Tucker [129], Deimling [30], Edmunds and Webb [34], Fitzpatrick [39], Hamilton [52], Goncharov [46], Grigorieff [50], Petryshyn [111], [114], Tucker [146], Wong [158], and others.

Using the Brouwer degree theory, Petryshyn and Tucker [129] obtained the following generalization of Theorem 1.2A.

TheOREM 1.2G. Let $D$ be a bounded open subset of $X$ with $0 \in D$. Suppose $T: \bar{D} \subset X \rightarrow X$ is $P_{\gamma}$-compact such that for some fixed $\mu$ dominating 
$\gamma$ the map $T$ satisfies conditions $\left(\pi_{\mu}^{\lessgtr}\right)$ and $(\Delta)$. Then the conclusions of Theorem 1.2A for equation (1.2-1) hold.

REMARK 1.2-2. In the actual statement of Theorem $1.2 \mathrm{G}$ in [129] it was assumed that $D$ is convex. However, the degree argument used in [129] to prove Theorem $1.2 \mathrm{G}$ never used the convexity of $D$. The latter was only used in deducing various special cases of Theorem $1.2 \mathrm{G}$ under various boundary conditions where sometimes the convexity of $D$ must be assumed.

Corollary $1.2 \mathrm{H}$. If $D$ is as in Theorem $1.2 \mathrm{G}$ and $T: \bar{D} \rightarrow X$ is $P_{1^{-}}$ compact and satisfies conditions $\left(\pi_{1}^{\leqq}\right)$and $(\Delta)$, then $T$ has a fixed point $x_{0}$ in $\bar{D}$; if $\left(\pi_{1}^{<}\right)$holds on $\partial D$, then the equation $T x-x=0$ is feebly approximation-solvable, and strongly approximation-solvable if $x_{0}$ is unique.

The existence part of Corollary $1.2 \mathrm{H}$ has also been reestablished by different arguments in [24], [46]. Corollary $1.2 \mathrm{H}$ holds, in particular, when $\left(\pi_{1}^{\leqq}\right)$is replaced by any one of the conditions in Remark $1.2-1$ if $D$ is convex.

Corollary $1.2 \mathrm{H}$ and the assertion in Example $1.1 \mathrm{f}$ imply the validity of the following result, assuming in the next corollary and in what follows that $X$ is a $\Pi_{1}$-space.

Corollary 1.2I. If $D$ is as in Theorem $1.2 \mathrm{G}$ and $T: \bar{D} \rightarrow X$ is ballcondensing and satisfies condition $\left(\pi_{1}^{\leqq}\right)$, then the conclusions of Corollary $1.2 \mathrm{H}$ hold since $T$ is $P_{1}$-compact.

We note that while the approximation-solvability aspect of Corollary $1.2 \mathrm{I}$ is new, its existence part follows from Proposition 1 in [122]. In case $D$ is also convex and $T(\bar{D}) \subset \bar{D}$, the existence result was first obtained by Sadovsky [136] for general $X$.

In [68] it was shown that if $G$ is a closed, bounded convex subset of $X$, $S$ contractive and $C$ compact on $G$, then $T=S+C$ has a fixed point in $G$ providing the following restrictive condition holds:

$$
S x+C y \in G \text { for all } x \text { and } y \text { in } G \text {. }
$$

The recent contributions by many authors (see [122] for the description of latest results and references) have extended the initial existence result of Krasnoselsky in various directions and to more general mappings, including those of semicontractive type introduced by Browder [12] (and Kirk [66] in a somewhat different manner).

In this section we examine some of these classes of mappings from the viewpoint of $A$-properness and $P_{\gamma}$-compactness and in that context outline some of the results obtained in [39], [15], [154], [158] while others will be discussed in Chapter 5 . 
Using Corollary $1.2 \mathrm{H}$, Proposition 1.1 , and the $P_{1}$-compactness of $T=I-S: X \rightarrow X$ established by the author in [110] whenever $S: X \rightarrow X$ is contractive, the following essentially constructive fixed point theorem was obtained in Petryshyn and Tucker [129] under very general and easily verifiable boundary conditions.

Corollary 1.2J. Let $D \subset X$ bounded and open with $0 \in D, C: \bar{D} \rightarrow X$ compact and $S: X \rightarrow X$ contractive. If $T=S+C$ satisfies condition $\left(\pi_{1}^{\leqq}\right)$, then $T$ has a fixed point $x_{0}$ in $\bar{D}$; if $\left(\pi_{1}^{<}\right)$holds on $\partial D$, then the equation

$$
S x+C x-x=0 \quad(x \in D)
$$

is feebly approximation-solvable, and strongly approximation-solvable if $x_{0}$ is unique.

For mappings $T$ of the form $T=S+C$, with $S$ contractive on all of $X$, Corollary $1.2 \mathrm{~J}$ appears to be the most general result from which one can obtain the constructive existence of a fixed point if one knows that $T$ has at most one fixed point in $D$. However, if $S$ is not defined on all of $X$, then even when $D=B(0,1)$ it is as yet unknown whether the mapping $I-S$ : $\bar{B}(0,1) \subset X \rightarrow X$ is $A$-proper when $S$ is contractive on $\bar{B}(0,1)$ and $X$ is a general $\Pi_{1}$-space.

It was shown in [129] and independently in [93] that when $S: \bar{B}(0, r) \rightarrow X$ is contractive with $l<\frac{1}{2}$, then $I-S: \bar{B}(0, r) \rightarrow X$ is $A$-proper. This follows from the fact that the radial retraction $R$ of $X$ onto $\bar{B}(0, r)$ is such that $\|R x-R y\| \leqq 2\|x-y\|$ for $x, y \in X$ and thus $S$ can be extended to a contraction on all of $X$. Consequently, Corollary $1.2 \mathrm{~J}$ remains valid in this case under the assumption that $S$ and $C$ are defined only on $\bar{B}(0, r)$ (see [114]). If $l \in\left[\frac{1}{2}, 1\right)$, then the $A$-properness of $I-S: \bar{B}(0, r) \rightarrow X$ takes place if either $X$ is reflexive and has a single-valued weakly continuous duality mapping (see [117]) or if $X$ has the so-called ball intersection property (see [96]). In particular, Hilbert spaces and $l_{p}$ spaces for $1<p<\infty$ have both weakly continuous duality map and ball intersection property.

In view of the above discussion the following analogue of Corollary 1.2J has been obtained in [114] as a corollary of a slightly more general Theorem 1 in [114].

Corollary $1.2 \mathrm{~K}$. Suppose $X$ is reflexive, $X^{*}$ strictly convex, and $J: X \rightarrow X^{*}$ is weakly continuous. If $S: \bar{B}(0,1) \rightarrow X$ is contractive, $C: \bar{B}(0,1) \rightarrow$ $X$ is compact and $T=S+C$ satisfies condition $\left(\pi_{1}^{\leqq}\right)$on $\partial B(0,1)$, then the conclusions of Corollary 1.2J hold.

It was shown by Browder [12] that if in Corollary $1.2 \mathrm{~K}$ one replaces the contractiveness assumption on $S: B \rightarrow X$ by the condition that $S$ be nonexpansive on $\bar{B}(0,1)$, then even when $X=l_{2}$ and $T=S+C$ maps $\vec{B}(0,1) \subset l_{2}$ into $\vec{B}(0,1)$, the mapping $T$ may fail to have a fixed point in 
$\bar{B}(0,1)$. Indeed, following Granas [48] define $S: \bar{B}(0,1) \subset l_{2} \rightarrow l_{2}$ and $C: \bar{B}(0,1) \rightarrow l_{2}$ by $S(x)=\left(0, x_{1}, x_{2}, \cdots\right)$ and $C(x)=\left(\sqrt{ }\left(1-\|x\|^{2}\right), 0,0, \cdots\right)$ and note that $S$ is nonexpansive, $C$ is compact, $T=S+C: \bar{B} \rightarrow \bar{B}$ but $T$ has no fixed point in $\bar{B}$. However, if one assumes that $C$ is completely continuous and $X$ is uniformly convex, then the existence of a fixed point for $T=S+C: D \rightarrow X$ has been established depending on the nature of $D$ and the conditions satisfied by $T$ on $\partial D$ (see [122] for latest results and references).

Although, under certain conditions on $X$, the theory of $P_{\gamma}$-compact and $A$-proper mappings has been used (see [34], [39], [114] and Chapter 5) to obtain existence of fixed points for mappings $T: \bar{B} \subset X \rightarrow X$ of the form $T=S+C$, where $S$ is nonexpansive and $C$ completely continuous on $\bar{B}$ or even for $T$ semicontractive, the constructive aspect of the theory is lost, that is, even when we know that $T$ has a unique fixed point in $B$ we cannot obtain it as a strong limit of solutions $x_{n} \in B_{n}$ of the approximate equation $T_{n}\left(x_{n}\right)=x_{n}$. In view of this, we will not dwell in this section on the existence results that are obtainable by means of the theory of $P_{\gamma^{-}}$ compact mappings. An interested reader should consult the original papers.

In [6] Belluce and Kirk introduced the notion of a generalized contraction which lies between contractive and nonexpansive mappings and which is defined to be a map $S: D \subset X \rightarrow X$ such that to each $x \in D$ there exists $\alpha(x) \in(0,1)$ such that $\|S x-S y\| \leqq \alpha(x)\|x-y\|$ for all $y$ in $D$. Extending the result of the writer [110], it was shown by Wong [158], using the argument of Kirk [66], that if $D=X$ and $S: X \rightarrow X$ is a generalized contraction, then $T=I-S$ is $P_{1}$-compact. In [42] Fitzpatrick established a similar result for generalized contractions defined only on closed convex subsets $D$ of $X$. However, he was not able to show the $A$-properness of $T: I-S: D \rightarrow$ $X$ for all points, but only at specific points. Nevertheless, the latter fact suffices to establish the constructive existence of fixed points of $S$ since a careful reading of the proof of Theorem 1 in [107] (i.e., Theorem 1.2A for $\mu=1$ ) reveals its validity under the assumption that $T$ be $P_{1}$-compact at 0 . To clarify this remark, the following definition has been used in [42] (see also Definition $3^{\prime}$ in [121] and [52]).

Definition 1.2L. $T: D \subseteq X \rightarrow Y$ is said to be A-proper at a given $g_{0} \in Y$ if any bounded sequence $\left\{x_{n_{j}}\right\}$ such that $x_{n_{j}} \in D_{n_{j}}$ and $T_{n_{j}}\left(x_{n_{j}}\right) \rightarrow g_{0}$ has a subsequence which converges to $x \in D$ and $T x=g_{0}$.

Using arguments similar to those in [66], the following lemma has been established in [42].

Lemma 1.2M. Let $X$ be a reflexive $\Pi_{1}$-space, $D \subset X$ closed and convex, $S: D \rightarrow X$ a generalized contraction and $g_{0} \in X$ such that $S(x)+g_{0} \in D$ for all $x$ in $D$. Then $T=I-S: D \rightarrow X$ is A-proper at $g_{0}$. 
Using Corollary $1.2 \mathrm{H}$ (with $T P_{1}$-compact at 0 ) and Lemma $1.2 \mathrm{M}$, Fitzpatrick proved in [42] the following

THEOREM 1.2N. Let $X$ be a reflexive $\Pi_{1}$-space, $D \subset X$ open, bounded and convex with $0 \in D$, and $S: \bar{D} \rightarrow \bar{D}$ a generalized contraction. Then $S$ has $a$ unique fixed point $x_{0} \in \bar{D}$ and if $x_{0} \notin \partial D$ then the equation $S x-x=0$ is uniquely approximation-solvable.

We add that the approximation-solvability result of Theorem $1.2 \mathrm{~N}$ is new but the existence part follows from a result of Kirk [66].

ReMARK 1.2-3. In view of a result in [158], Corollary $1.2 \mathrm{~J}$ remains valid if we weaken the condition on $S: X \rightarrow X$ to that of being a generalized contraction but strengthen the condition on $X$ to that of being reflexive.

Instead of considering the sum $S+C$ of the above mappings we may consider mappings formed by intertwining of mappings of the above type but with a stronger assumption on the second variable. Indeed, let $X$ be reflexive and $D \subset X$ bounded, open and convex. Following Kirk [66] we say $T: \bar{D} \rightarrow X$ is strongly semicontractive if there exists a mapping $V: X \times$ $\bar{D} \rightarrow X$ such that $T(x)=V(x, x)$ for $x \in \bar{D}$ and

(i) for each $y \in \bar{D}, V(\cdot, y): X \rightarrow X$ is a generalized contraction.

(ii) if $\left\{x_{n}\right\} \subset X$ is bounded and $\left\{y_{n}\right\} \subset \bar{D}$ such that $y_{n} \rightarrow y_{0}$, then $V\left(x_{n}, y_{n}\right)-V\left(x_{n}, y_{0}\right) \rightarrow 0$.

The following constructive version of the fixed point theorem in [66] has been obtained in [39], [158] by using Corollary $1.2 \mathrm{H}$ and the $P_{1^{-}}$ compactness of $T$.

THEOREM 1.20. If $X$ and $D$ satisfy the above conditions and $T: \bar{D} \rightarrow X$ is strongly semicontractive and satisfies the corresponding conditions on $\partial D$, then the conclusions of Corollary $1.2 \mathrm{H}$ hold.

In [15] Browder obtained an existence result similar to the above theorem, where $X$ was assumed to be uniformly convex while the condition (i) was replaced by the assumption that $V(\cdot, y): X \rightarrow X$ is nonexpansive for each $y$ in $\bar{D}$. For further results for intertwining mappings see [12], [42], [66], [154] and Chapter 5.

REMARK 1.2-4. The following observation is perhaps of some interest. It follows from Krasnoselsky's result that if $D$ is an open, bounded, convex set in $X$ with 0 in $D, S$ contractive on $\bar{D}$ and $C$ compact on $\bar{D}$ such that condition (K) holds, then there exists $x_{0} \in \bar{D}$ such that $x_{0}=$ $S x_{0}+C x_{0}$. To the best of my knowledge there is no way of constructing this fixed point even when we know that $x_{0}$ lies in $D$ and is unique. However, if we assume that $X$ is a reflexive $\Pi_{1}$-space and $C$ is completely continuous on $\bar{D}$, then it is easy to show directly that, in view of condition (K), $T=S+C: \bar{D} \rightarrow X$ is $P_{1}$-compact at 0 . Since $T$ maps $\bar{D}$ into $\bar{D}$ and $\bar{D}$ is 
convex, $T$ satisfies condition $\left(\pi_{1}^{\lessgtr}\right)$ on $\partial D$. Consequently, the conclusions of Corollary 1.2H hold; and if $x_{0} \in D$ is a unique fixed point of $T$, then the solutions $x_{n} \in D_{n}$ of $T_{n}\left(x_{n}\right)=x_{n}$ are such that $x_{n} \rightarrow x_{0}$, i.e., a fixed point of $T=S+C$ can, in fact, be constructed.

Other fixed point theorems which are known to be true for compact maps have been extended to $P$-compact mappings (see [31], [114], [146]). It should be noted, in particular, that the Leray-Schauder fixed point theorem involving a family $C(x, t): \bar{D} \times[0,1] \rightarrow X$ of continuous compact mappings has been extended in [146] and under different conditions in [34] to a family $C(x, t)$ of $P$-compact mappings without the use of the degree theory (see [34], [146] for details).

Structure of fixed point sets. Suppose $D$ is a given subset of $X$ and $T: D \rightarrow X$ is a suitable continuous mapping with a nonempty set of fixed points $F(T) \subset D$. If $F(T)$ does not consist of a single point, the question when is $F(T)$ a continuum (i.e., nonempty, compact and connected set) arises. In the case of compact, proper, and $k$-set contractive mappings on general Banach spaces this question has been investigated by Aronszajn, Stampacchia, Krasnoselsky and Sobolevsky, Browder and Gupta, Vidossich, and Petryshyn (see [42], [153] for earlier literature). For $\boldsymbol{P}_{\mathbf{1}^{-}}$ compact and $A$-proper mappings this question was studied by Deimling [30] and Fitzpatrick [39], [42].

If it cannot be assured that a given operator has a unique fixed point, the general idea is to approximate this operator by more "regular" ones in a sense which will be made precise below. In this section we present the first theorem of Deimling [30] which provides sufficient conditions for $F(T)$ of a $P_{1}$-compact map $T$ to be a continuum. The interesting feature of the proof of this theorem is that it uses only Corollary $1.2 \mathrm{H}$ and no degree theory.

THEOREM 1.2P. Let $D \subset X$ be a bounded, open and convex set with $0 \in D$, $T: \bar{D} \rightarrow X$ a continuous $P_{1}$-compact map such that $T(\partial D)$ is bounded and the boundary condition $\left(\pi_{1}^{<}\right)$is satisfied. Suppose $\left\{T^{n}\right\}$ is a sequence of continuous $P_{1}$-compact mappings of $\bar{D}$ into $X$ such that:

(R1) $\delta_{n}=\sup \left\{\left\|T^{n} x-T x\right\|: x \in \bar{D}\right\} \rightarrow 0$ as $n \rightarrow \infty$.

(R2) The equation $x=T^{n} x+f$ has at most one solution if $\|f\| \leqq \delta_{n}$.

Then the set $F(T)$ is a continuum.

An immediate corollary of Theorem 1.2P is the following result.

CoROllary 1.2Q. Suppose $T: \bar{D} \rightarrow X$ is a nonexpansive $P_{1}$-compact mapping which satisfies condition ( $\leqq$ ) on $\partial D$. Then $F(T)$ is a continuum.

By using the degree theory for continuous $A$-proper mappings, Theorem 1.2P was extended to the case when $D$ was no longer assumed to be convex. 
This second theorem together with some of its corollaries will be discussed in Chapter 4 in conjunction with analogous but more general results obtained by Fitzpatrick [42].

Positive $P_{1}$-compact operators. Let $K$ be a cone in $X$ and introduce a partial ordering in $X$ which is compatible with the vector structure of $X$ by letting $x \leqq y$ if and only if $y-x \in K$. Hence $x \leq y$ means that $y-x \notin K$. An operator $T: D \subset X \rightarrow X$ is said to be positive if $T: D \cap K \rightarrow K$. Note that $K_{n}=K \cap X_{n}$ is a cone in $X_{n}$ and we assume that the scheme $\Gamma_{0}=\left\{X_{n}, P_{n}\right.$; $\left.X_{n}, P_{n}\right\}$ for $(X, X)$ is such that $P_{n}(K) \subseteq K_{n}$ for $n \geqq n_{0}$.

We present two fixed point theorems for positive $P_{1}$-compact mappings extending the corresponding results in Krasnoselsky [10] for positive compact maps.

TheOREM 1.2R. Let $B_{K R}=\bar{B}(0, R) \cap K$ and let $T: B_{K R^{\prime}} \rightarrow K$ be $P_{1^{-}}$ compact at 0 (i.e., $T_{\lambda}=T-\lambda I: B_{K R} \rightarrow K$ is $A$-proper at 0 for $\lambda \geqq 1$ ). Suppose that the following conditions are satisfied:

( $\left.\pi_{1}^{\lessgtr}\right)$ If $T x=\alpha x$ for some $x \in \partial B_{K R}$ then $\alpha \leqq 1$.

( $\Delta)$ If $P_{n} T x=\lambda x$ for some $x \in \partial_{K R} \cap X_{n}$ and $n$, then $\lambda \leqq c$ for $c>0$ independent of $n$.

Then $T$ has a fixed point $x_{0}$ in $B_{K R}$. If $\left(\pi_{1}^{<}\right)$holds, then the equation

$$
T x-x=0 \quad\left(x \in B_{K R}\right)
$$

is feebly approximation-solvable in $B_{K R}$, and strongly approximationsolvable if $x_{0}$ is unique.

Note that Theorem 1.2R is valid for the case when $T$ is ball-condensing and, in particular, when $T$ is compact or of the form $T=S+C$ with $S: X \rightarrow X$ contractive and $C$ compact. Condition $\left(\pi_{1}^{5}\right)$ is implied by any one of the conditions: (S1) $T x \geq(1+\varepsilon) x$ for all $\varepsilon>0$ and $x \in \partial B_{K R}$; (S2) $T x \geq x$ for $x \in \partial B_{K R}$; (S3) $\|T x-x\|^{2} \geqq\|T x\|^{2}-\|x\|^{2}$ for $x \in \partial B_{K R}$; (S4) $(T x, J x) \leqq(x, J x)$ for $x \in \partial B_{K R}$.

In dealing with positive operators one is often interested in obtaining nonzero fixed points. Assuming somewhat stronger conditions on $T$ one obtains the generalization of the corresponding results in [40] for the existence of nontrivial fixed points.

THEOREM 1.2S. Let $r$ and $R$ be positive real numbers, $r \neq R, r^{*}=$ $\min \{r, R\}$ and $R^{*}=\max \{r, R\}$. Let $B_{K}^{*}=\left\{x \in K \mid\|x\|<R^{*}\right\}$ and $U^{*}=$ $\left\{x \in K \mid r^{*}<\|x\|<R^{*}\right\}$. Let $T: \bar{B}_{K}^{*} \rightarrow K$ be a bounded $P_{1}$-compact mapping such that

(T1) If $T x=\alpha x$ for some $x$ in $\partial B_{K R}$, then $\alpha<1$.

(T2) There exists $h_{0} \in K$ with $h_{0} \neq 0$ such that $x-T x \neq \beta h_{0}$ for $x \in \partial B_{K R}$ and $\beta \geqq 0$. 
Then equation (1.2-4) is feebly approximation-solvable in $U^{*}$, and strongly approximation-solvable in $U^{*}$ if it is uniquely solvable in $U^{*}$.

REMARK 1.2-5. The existence part of Theorem $1.2 \mathrm{~S}$ was proved by Goncharov [46] for the case when $T: K \rightarrow K$ is a bounded $P$-compact mapping. Using the retraction argument, Brouwer fixed point theorem, and a device used in [70], Goncharov [46] also proved the existence part of Theorem 1.2S assuming that (T1) holds for $\alpha \leqq 1$ and that (T2) holds for all $\beta>0$. Note that condition (T2) is implied by condition (S2). In this case one obtains the existence of a fixed point $x_{0}$ of $T$ such that $r^{*} \leqq$ $\left\|x_{0}\right\| \leqq R^{*}$. Later, but independently, the existence of a fixed point $x_{0}$ in $U^{*}$ under conditions (T1) and (T2) was proved by Hamilton [52] by using retraction and the Brouwer degree argument. Theorem 1.2S includes the corresponding result for compact maps in [70] and ballcondensing maps. A similar result has been obtained by Nussbaum for set-condensing maps.

\section{Equations involving linear $A$-proper mappings}

The first part of Chapter 2 contains some basic results concerning the relationship between the $A$-properness of a bounded linear mapping $T: X \rightarrow Y$ and the unique approximation-solvability of the equation

$$
T(x)=f \quad(f \in Y) .
$$

The theory of $A$-proper mappings is then used to deduce as special cases the earlier results of a number of authors concerning the convergence of various methods of Galerkin type used in the solvability of equation (2.1) involving bounded and unbounded mappings. The Fredholm alternative for equation (2.1) involving $A$-proper mappings is also discussed. The second part of Chapter 2 contains essentially new observations on the relation of the $A$-properness of $T$ to the stability of the approximation method

$$
T_{n}(x)=Q_{n} f \quad\left(x \in X_{n}, T_{n}=\left.Q_{n} T\right|_{X_{n}}\right)
$$

in the sense of Mikhlin [88] and on the applications of the theory of $A$ proper mappings to the Dirichlet boundary value problem for elliptic equations.

2.1. Approximation-solvability and bounded A-proper mappings. In what follows we denote by $L(X, Y)$ the space of bounded linear mappings from $X$ to $Y$. Unless stated otherwise, we assume that $(X, Y)$ is a pair of Banach spaces provided with a projectionally complete scheme $\Gamma=$ $\left\{X_{n}, P_{n} ; Y_{n}, Q_{n}\right\}$ and that $T \in L(X, Y)$. 
Our first result is the following theorem established by the author in [113] which characterizes the class of bounded linear mappings and which initially motivated the study of $A$-proper mappings.

THEOREM 2.1A. Equation (2.1) is uniquely approximation-solvable for each $f$ in $Y$ if and only if $T$ is A-proper and one-to-one.

REMARK 2.1-1. The key observation to be made about the proof of Theorem 2.1A is that it does not use the notion of the adjoint $T^{*}$ which is a highly linear concept. Consequently, as we shall see in Chapter 3 , the same type of argument used in the theory of the approximation-solvability and/or solvability of equations involving linear $A$-proper mappings carries over to equations involving nonlinear $A$-proper mappings.

Theorem 2.1A and Example 1.1a show that unless $T$ is $A$-proper the solutions $x$ of equation (2.1) cannot be obtained as strong limits of solutions $x_{n} \in X_{n}$ of equation (2.2) for a given $\Gamma$ even when we know that equation (2.1) is uniquely solvable for each $f$ in $Y$.

THEOREM 2.1B. T is A-proper and one-to-one if and only if the following two conditions hold:

(2.1-1) There exist a constant $c>0$ and an integer $n_{0} \geqq 1$ such that $\left\|T_{n}(x)\right\| \geqq c\|x\|$ for all $x \in X_{n}$ and all $n \geqq n_{0}$.

(2.1-2) If $\left\{x_{n_{j}} \mid x_{n_{j}} \in X_{n_{j}}\right\}$ is any bounded sequence such that $T_{n_{j}}\left(x_{n_{j}}\right) \rightarrow g$ for some $g$ in $Y$, then there exists $x \in X$ such that $T(x)=g$.

It is not hard to show (see [113]) that as a consequence of Theorem 2.1B one obtains the following result which provides some practical means to verify that a given mapping $T \in L(X, Y)$ is $A$-proper.

THEOREM 2.1C. Suppose that any one of the following three conditions hold:

(2.1-3) $R(T)=Y$.

(2.1-4) $Q_{n} Q_{m}=Q_{n}$ for $m \geqq n$ and $X$ is reflexive.

(2.1-5) $Q_{n}^{*} u \rightarrow u$ in $Y^{*}$ for each $u$ in $Y^{*}$.

Then $T$ is A-proper and one-to-one if and only if condition (2.1-1) holds.

We add in passing that (2.1-5) holds if $\Gamma=\Gamma_{s}$ and $Y$ has a shrinking Schauder basis (see Marti [83]), and in particular if $Y$ has a Schauder basis and is reflexive.

It will be seen below that Theorems $2.1 \mathrm{~A}, 2.1 \mathrm{~B}$, and $2.1 \mathrm{C}$ include the convergence results of Kantorovich [63], Mikhlin [86], [87], Polsky [131], [132], Sobolevsky [143], Medvedev [85], Martyniuk [84], the writer [102], [103], [104], and other authors for the methods of Galerkin type involving both bounded and unbounded operators. We will illustrate this assertion with the following discussion for the case of bounded maps. 
Galerkin method. Let $C \in L(X, X)$ be compact. The Galerkin method for the solvability of the equation

$$
x-C x=f \quad(f \in X)
$$

consists of solving the finite dimensional equations

$$
x_{n}-P_{n} C x_{n}=P_{n} f
$$

in order to determine the approximate solution $x_{n} \in X_{n}$. In this general setting the convergence of the method was first obtained by Kantorovich [63] and Mikhlin [87]. Since $T=I-C$ is $A$-proper with respect to $\Gamma_{0}=$ $\left\{X_{n}, P_{n} ; X_{n}, P_{n}\right\}$, their result follows as the following corollary of Theorem 2.1A for the case when $Y=X, Y_{n}=X_{n}$, and $Q_{n}=P_{n}$.

COROLlaRY 2.1D. If $T=I-C$ is one-to-one, then equation (2.1-6) is uniquely approximation-solvable for each $f \in X$.

Another corollary of Theorem 2.1A which includes the results established in [55], [85], [103], [131] for the case when $X$ is a Hilbert space is the following.

COROLlaRY 2.1E. Let $X$ be a reflexive $\Pi_{1}$-space with $X^{*}$ strictly convex and let $J$ be the normalized duality map of $X$ into $X^{*}$. Suppose $T \in L(X, X)$ is such that any one of the following three conditions hold:

(2.1-8) $(T x, J x) \geqq c\|x\|^{2}$ for all $x \in X$ and some $c>0$.

(2.1-9) $T$ is one-to-one and $\lim _{\inf _{n \rightarrow \infty}}\left(T x_{n}, J x_{n}\right) \geqq \delta>0$ whenever $x_{n} \rightarrow 0$ with $\left\|x_{n}\right\|=1$ for all $n$.

(2.1-10) $T=A+C, T$ is one-to-one, $C$ is compact and $A$ satisfies either (2.1-8) or (2.1-9).

Then equation (2.1) is uniquely approximation-solvable with respect to $\Gamma_{0}$ for each $f \in X$.

Petrov-Galerkin method. Let $\left\{X_{n}\right\}$ and $\left\{Y_{n}\right\}$ be two distinct sequences in $X$ so that $\Gamma_{1}=\left\{X_{n}, P_{n} ; Y_{n}, Q_{n}\right\}$ is projectionally complete for $(X, X)$. The generalization of the Galerkin method suggested in [99] consists in constructing a solution $x$ of equation (2.1-6) as a limit of solutions $x_{n} \in X_{n}$ of the equations

$$
Q_{n} x_{n}-Q_{n} C x_{n}=Q_{n} f \quad\left(x_{n} \in X_{n}, Q_{n} f \in Y_{n}\right)
$$

which are different from equation (2.1-7) since $Q_{n} x_{n} \neq x_{n}$. In this abstract setting and under the assumption that equation (2.1-6) is uniquely solvable for each $f$ in $X$, Polsky [132] established the unique approximationsolvability of equation (2.1-6) with respect to $\Gamma_{1}$ for each $f$ in $X$ under the following. 
Condition (A). There exist $c_{0}>0$ and $n_{0} \geqq 1$ such that $\|y\| \leqq c_{0}\left\|Q_{n} y\right\|$ for all $y$ in $X_{n}$ and all $n \geqq n_{0}$.

It was observed by Polsky that Condition (A) is also a necessary condition for equation (2.1-6) to be uniquely approximation-solvable with respect to $\Gamma_{1}$ for each $f$ in $X$ in the sense that if Condition (A) does not hold then one can give examples of $C$ and $f$ for which equation (2.1-11) is either not solvable or has infinitely many solutions or the approximate solutions $x_{n}$ do not converge. In [149] Vainikko has shown directly even for a more general setting that Condition (A) is both necessary and sufficient for the convergence of the Petrov-Galerkin method.

It turns out that the basic result of Polsky can be easily imbedded into the general theory of $A$-proper mappings. It follows from Theorem 2.1A and the following lemma deduced from Theorem 2.1C.

Lemma 2.1F. Suppose $C \in L(X, X)$ is compact. Then $T=I-C$ is $A$-proper with respect to $\Gamma_{1}$ if and only if Condition (A) holds.

Proof. Suppose $T$ is $A$-proper with respect to $\Gamma_{1}$. Then, by Proposition 1.1D, the identity $I=T+C$ is $A$-proper with respect to $\Gamma_{1}$. If there were a sequence $\left\{x_{n_{j}} \mid x_{n_{j}} \in X_{n_{j}}\right\}$ such that $\left\|x_{n_{j}}\right\|=1$ and $Q_{n_{j}} x_{n_{j}} \rightarrow 0$, then $I$ would not be $A$-proper with respect to $\Gamma_{1}$. Hence there exist $c>0$ and $n_{0} \geqq 1$ such that $\left\|Q_{n} x\right\| \geqq c\|x\|$ for $x \in X_{n}$ and $n \geqq n_{0}$, that is, Condition (A) holds. Suppose now that Condition (A) holds. Since $I$ is onto, Theorem 2.1C shows that $I$ is $A$-proper with respect to $\Gamma_{1}$. Hence, by Proposition 1.1D, $T=I-C$ is $A$-proper with respect to $\Gamma_{1}$. Q.E.D.

An analogous characterization of the unique approximation-solvability of Polsky obtained in [131] for equation (2.1) with $T \in L(H, H)$ is deduced even for $T \in L(X, X)$ from Theorem 2.1A and the following consequence of Theorem 2.1C.

Lemma 2.1G. Suppose $T \in L(X, X)$ is bijective and $\tau_{n}=\inf \left\{\left\|P_{n} z\right\| \mid z \in\right.$ $\left.T\left(X_{n}\right),\|z\|=1\right\}>0$. Then $T$ is $A$-proper with respect to $\Gamma_{0}=\left\{X_{n}, P_{n} ; X_{n}, P_{n}\right\}$ if and only if Condition (A), $\lim \inf _{n} \tau_{n}=\tau>0$, holds.

The following special case of Theorem 5 in the author's paper [119] will prove to be useful in $\$ 2.2$.

LeMma 2.1H. Let $H$ and $H_{0}$ be Hilbert spaces with $H_{0} \subset H$ such that $H_{0}$ is dense in $H$ and the injection of $H_{0}$ into $H$ is continuous. Let $T, K \in$ $L\left(H_{0}, H\right)$ and let $\Gamma_{0}=\left\{X_{n}, P_{n} ; Y_{n}, Q_{n}\right\}$ be projectionally complete for $\left(H_{0}, H\right)$ and such that $Q_{n} K x=K x$ for each $x \in X_{n}$ and each $n$. If there exists a compact map $C \in L\left(H_{0}, H_{0}\right)$, constants $a>0$ and $b \geqq 0$, and an integer $n_{0} \geqq 1$ such that for each $n \geqq n_{0}$

$$
(T x, K x) \geqq a|x|_{0}^{2}-(C x, x)_{0} \text { for all } x \in X_{n},
$$

then $T: H_{0} \rightarrow H$ is $A$-proper with respect to $\Gamma_{0}$. 
2.2. Equations involving unbounded linear operators. In this section we show how the theory of $A$-proper mappings outlined in $\$ 2.1$ can be used to study the approximation-solvability of equations involving various classes of unbounded linear operators. The relation of results presented here to those of other authors will be indicated. The basic virtue of the theory of $A$-proper mappings as used here lies not only in the fact that it presents a general and a unified approach to problems treated differently by a number of other authors but, as will be seen later, it presents a somewhat new approach to the approximation-solvability of equations involving abstract and differential equations.

Equations with $K$-p.d. operators. Let $H$ be a real Hilbert space and let $A$ and $K$ be linear operators such that $D(A)$ is dense in $H$ and $K$ is closeable with $D(K) \supseteq D(A)$ and $K D(A)$ dense in $H$. The map $A: D(A) \rightarrow H$ is called $K$-positive definite $\left(K\right.$-p.d.) if there are constants $\alpha_{1}>0$ and $\alpha_{2}>0$ such that

$$
(A x, K x) \geqq \alpha_{1}\|x\|^{2} \text { and }\|K x\|^{2} \leqq \alpha_{2}(A x, K x) \text { for } x \in D(A) ;
$$

$A$ is called $K$-symmetric if $(A x, K y)=(K x, A y)$ for $x, y \in D(A)$. Note that the class of $K$-p.d. and $K$-symmetric operators contains among others: (a) positive definite and symmetric operators (when $K=I$ ); (b) closeable invertible operators (when $K=T$ ); (c) certain ordinary and partial differential operators of odd and even order (see [82], [84], [141]); (d) bounded symmetrizable operators (see [102]), and others.

Suppose that $A$ is both $K$-p.d. and $K$-symmetric and let $H_{0}$ denote the completion of $D(A)$ in the metric

$$
[x, y]=(A x, K y), \quad|x|=[x, x]^{1 / 2} \quad(x, y \in D(A)) .
$$

Then it is known [102], [104] that $H_{0}$ can be regarded as a subset of $H$, the mapping $x \rightarrow K x$ of $D(A)$ into $H$ is bounded from the $H_{0}$-norm to $H$ and so can be extended uniquely to a bounded linear map $K_{0}$ of $H_{0}$ into $H$ and $D\left(K_{0}\right)=H_{0} \subseteq D(R)$ where $R$ is the closure of $K$ in $H$; moreover, $A$ has a unique closed $K_{0}$-p.d. and $K_{0}$-symmetric extension $A_{0}$ with $D\left(A_{0}\right)$ in $H_{0}$ such that $A_{0}: D\left(A_{0}\right) \rightarrow H$ is bijective. We refer to $A_{0}$ as the Friedrichs extension of $A$ and note that for $K=I$ the map $A_{0}$ furnishes the selfadjoint extension constructed by Friedrichs (see [43], [86]).

It was shown in [104] that one can use $A_{0}$ to construct the solvable extension for a given $K$-p.d. but not necessarily $K$-symmetric operator $L$ and then use this fact in the approximation-solvability of the equation

$$
L x=f .
$$

Thus the following extension of the results of Friedrichs and Lax and 
Milgram [76] is useful (see the author's paper [104] for further results and proofs).

THEOREM 2.2A. Let $A$ be K-p.d. and K-symmetric and let $L$ be a linear operator with $D(L)=D(A)$ such that for some $\eta_{1}>0$ and $\eta_{2}>0$

$$
\begin{gathered}
(L x, K x) \geqq \eta_{1}|x|^{2} \quad \text { for } x \in D(L), \\
|(L x, K y)| \leqq \eta_{2}|x||y| \quad \text { for } x, y \in D(L) .
\end{gathered}
$$

Then $L$ has a unique closed extension $L_{0}$ of the form $L_{0}=A_{0} W_{0}$ with $D\left(L_{0}\right) \subset H_{0}$ such that $L_{0}: D\left(L_{0}\right) \rightarrow H$ is bijective, $L_{0}$ satisfies (2.2-4) for $x \in D\left(L_{0}\right)$ and (2.2-5) for $x \in D\left(L_{0}\right)$ and $y \in H_{0}$ with $K_{0}$ replacing $K$, and $W_{0}$ is the extension of the bounded map $W=A_{0}^{-1} L: D(L) \rightarrow H_{0}$ such that $W \subset W_{0} \subset \bar{W}$ and $R\left(W_{0}\right)=D\left(A_{0}\right)$.

Note that the closure $\bar{W}: H_{0} \rightarrow H_{0}$ exists and is such that

$$
[\bar{W} x, x] \geqq \eta_{1}|x|^{2} \quad \text { for } x \in H_{0} .
$$

We add that the interesting feature of the proof of Theorem $2.2 \mathrm{~A}$ is that it depends only on the linear operator $A_{0}$ and the bijectivity of $\bar{W}$ (and not on the linearity of $L$ ). Consequently, as has been shown in [105], Theorem 2.2A remains valid for densely defined nonlinear operators satisfying generalized monotonicity conditions.

As a corollary of Theorem $2.2 \mathrm{~A}$ and Theorem 2 in [104] we obtain the following result which will be useful in the solvability of boundary value problems.

Corollary 2.2B. Let $A$ be $K$-p.d. and $K$-symmetric and suppose that $K$ is closed and that $D(K)=D(A)$. If $L$ is a closeable operator for which (2.2-4) holds for all $x$ in $D(L)=D(A)$, then $L_{0}=L$ and $H_{0}=D(L)$.

REMARK 2.2-1. The extension results mentioned above are valid for Hilbert spaces which need not be separable or real. In case $H$ is complex, (2.2-4) is replaced by $|(L x, K x)| \geqq \eta|x|^{2}$ for $x \in D(L)$ and the $K$-symmetry of $A$ follows from the fact that $A$ is $K$-p.d.

The above results are useful in the study of the approximation-solvability of equations involving operators acting in separable Hilbert spaces.

Let $A: D(A) \subset H \rightarrow H$ be $K$-p.d. and $K$-symmetric. Let $H_{0}$ be the space constructed above. Our problem is to study the approximation-solvability of the equation

$$
L x+M x=f \quad(f \in H),
$$

where $L$ satisfies the conditions of Theorem $2.2 \mathrm{~A}$ and $M$ is such that $D(M) \supseteq D(L)$ and $A_{0}^{-1} M: D(A) \subset H_{0} \rightarrow H_{0}$ is bounded. It is easy to show 
that if $\left\{\phi_{i}\right\} \subset H_{0}$ is linearly independent and complete in $H_{0}$, then $\left\{K \phi_{i}\right\}$ is linearly independent and complete in $H$. If $X_{n}=\left[\phi_{1}, \cdots, \phi_{n}\right], Y_{n}=$ $\left[K \phi_{1}, \cdots, K \phi_{n}\right], P_{n}: H_{0} \rightarrow X_{n}$ and $Q_{n}: H \rightarrow Y_{n}$ are orthogonal projections in $H_{0}$ and $H$ respectively, then $\Gamma^{0}=\left\{X_{n}, P_{n} ; Y_{n}, Q_{n}\right\}$ is projectionally complete for $\left(H_{0}, H\right)$.

The generalized moments method. Assuming that $\left\{\phi_{i}\right\} \subset D(L)$, the GM method consists in finding $x_{n}=\sum_{i=1}^{n} a_{i}^{n} \phi_{i} \in X_{n}$ from the condition that $L x_{n}+M_{n} x-f$ be orthogonal to $\left\{K \phi_{i}\right\}, 1 \leqq i \leqq n$, that is, from

$$
\sum_{i=1}^{n}\left\{\left(L \phi_{i}, K \phi_{j}\right)+\left(M \phi_{i}, K \phi_{j}\right)\right\} a_{i}^{n}=\left(f, K \phi_{j}\right), \quad 1 \leqq j \leqq n
$$

The system $(2.2-8)$ can be written in the form

$$
Q_{n} L x_{n}+Q_{n} M x_{n}=Q_{n} f .
$$

It follows easily that (2.2-9) can be written in the form

$$
P_{n} W x_{n}+P_{n} A_{0}^{-1} M x_{n}=P_{n} A_{0}^{-1} f
$$

or in the form

$$
P_{n} \bar{W} x_{n}+P_{n} C x_{n}=P_{n} A_{0}^{-1} f,
$$

where $\bar{W}$ and $C$ are the closures in $H_{0}$ of $W$ and $A_{0}^{-1} M$ respectively. Thus the GM method reduces to the method of Galerkin for the equation

$$
\bar{W} x+C x=A_{0}^{-1} f \quad\left(x \in H_{0}, A_{0}^{-1} f \in H_{0}\right) .
$$

It is clear that every solution of (2.2-7) is a solution of (2.2-12) but the converse is not true in general. However, if $D(M) \supseteq H_{0}$ then, by the fact that $L_{0}=A_{0} W_{0}$ with $R\left(W_{0}\right)=D\left(A_{0}\right)$, it can be shown that in this case $x_{0} \in D\left(L_{0}\right)$. In what follows we regard a solution $x_{0} \in H_{0}$ of (2.2-12) as a generalized solution of equation (2.2-7). If we assume that $\left\{\phi_{i}\right\}$ lie in $\boldsymbol{H}_{0}$ but not in $D(L)$, then equation (2.2-12) must be used for the construction of a solution (ordinary or generalized) of equation (2.2-7).

If we assume that $C$ is compact, then in view of (2.2-6), the map $\bar{W}+$ $C: H_{0} \rightarrow H_{0}$ is $A$-proper with respect to $\Gamma_{0}=\left\{X_{n}, P_{n} ; X_{n}, P_{n}\right\}$ and thus Theorem 2.1A implies the following basic result for the GM method which, as will be seen, contains most of the earlier results for the projective type methods.

TheOREM 2.2C. If $C$ is compact and $\bar{W}+C$ is one-to-one then there exists an integer $n_{0} \geqq 1$ such that equation (2.2-8) or (2.2-9) has a unique solution $x_{n} \in X_{n}$. Moreover, $x_{n} \rightarrow x_{n}$ in $H_{0}$ and $x_{0}$ is the unique solution (possibly generalized) of equation (2.2-7). 
In view of Lemma $2.1 \mathrm{H}$, Theorem $2.2 \mathrm{C}$ follows as a corollary of the following new result which is more useful in applications at least in those cases when the Sobolev type theorems are applicable.

THEOREM 2.2D. Suppose that all the conditions of Theorem 2.2C are satisfied except for the inequality (2.2-4) which is replaced by

Condition (a): There exists a compact map $C_{0}: D(L) \subset H_{0} \rightarrow H_{0}$ such that $(L x, K x) \geqq \eta_{3}|x|^{2}-\eta_{4}\left[C_{0} x, x\right]$ for all $x$ in $D(L)$ and some $\eta_{3}>0$ and $\eta_{4} \geqq 0$.

Then the conclusions of Theorem $2.2 \mathrm{C}$ hold.

Let us remark that if in Theorem $2.2 \mathrm{C}$ the map $M \equiv 0$, then by virtue of Theorem 2.2A the generalized solution $x_{0}$ constructed by Theorem $2.2 \mathrm{C}$ does lie in $D\left(L_{0}\right)$. However, if $M \neq 0$, then in general the generalized solution $x_{0} \in H_{0}$ of (2.2-7) constructed by Theorem $2.2 \mathrm{C}$ need not lie in $D\left(L_{0}\right)$. Moreover, unless $L$ is of a very special type and $\left\{\phi_{i}\right\}$ are chosen in a special way (see [32], [86]) the residual error $\left\|L x_{n}+M x_{n}-f\right\|$ will not converge to 0 . The next new theorem shows how both of these deficiencies can be eliminated if we strengthen the conditions on $L$ and $K$. It also has the interesting feature that the theory of $A$-proper mappings is applicable directly to equation (2.2-7) without using the auxiliary equation (2.2-11).

THeOREM 2.2E. Suppose $A$ is $K$-p.d. and $K$-symmetric with $K$ closed and $D(K)=D(A)$. Suppose $L$ is a closeable operator with $D(L)=D(A)$ for which either the inequality (2.2-4) or Condition (a) holds. Suppose further that $M$ is $H_{0}$-compact (i.e., $M$ is compact as a map from $H_{0}$ to $H$ ). Then $L+M: D(L) \subset H_{0} \rightarrow H$ is A-proper with respect to $\Gamma^{0}$ and if $L+M$ is also one-to-one, then equation (2.2-7) is uniquely approximation-solvable with respect to $\Gamma^{0}$ for each $f \in H$. Moreover, $x_{0} \in D(L)$ and $\left\|L x_{n}+M x_{n}-f\right\| \rightarrow 0$ as $n \rightarrow \infty$, where $x_{n}$ is a solution of equation (2.2-8).

Proof. We indicate the proof for the more general case when $L$ satisfies Condition (a). It follows from Theorem 2 in [104] that $K_{0}=K$, $A_{0}=A$, and $H_{0}=D(A)$ with $|x|,\|T x\|$ and $\|K x\|$ providing equivalent norms on $D(A)$. This and the closed graph theorem imply that $L: H_{0} \rightarrow H$ is bounded. In view of Condition (a), Lemma $2.1 \mathrm{H}$ implies that $L: H_{0} \rightarrow H$ is $A$-proper with respect to $\Gamma^{0}$ and so is $L+M$ since $M: H_{0} \rightarrow H$ is compact. Now, the first conclusion of Theorem 2.2E follows from Theorem 2.1A. The last assertion follows from the fact that $H_{0}=D(L)$ and $L, M: H_{0} \rightarrow H$ are bounded. Q.E.D.

Special cases. The following are the most important special cases of the GM method formulated and studied earlier by a variety of principles and methods.

(i) Galerkin method. When $K=I$ and $L=A$, then $A_{0}$ is selfadjoint and positive definite, $H_{0}$ is the energy space $H_{A}, \bar{W}=I$ and the GM method 
reduces to the well-known Galerkin method. In this abstract form Theorem 2.2C was first proved by Mikhlin [86], although beginning with Galerkin the method has been extensively used earlier in the study of the solvability of differential equations (see [72], [73], [86] for numerous references). For more recent study of the method see [26], [132], [149], [152].

(ii) The method of moments. When $L=A$ and $K=A$, then $H_{0}$ is the space with the scalar product $[u, v]=(A u, A u)$ and the GM method reduces to the method of moments whose study was initiated by Krilov and Kravchuk (see [73]) and further studied by a number of authors (e.g. [74], [84], [102]). In its present form Theorem $2.2 \mathrm{C}$ was proved by the author [102]. If $L=A$ and $K \neq A$, then $L_{0}$ is $K$-p.d. and $K$-symmetric and the method has been studied by Martyniuk [84] (see also [75]) for special classes and by the author [102] in its present setting. If $L \neq A$ and $K \neq A$, the GM method was proved in [109]. The GM method is related to the GalerkinPetrov method initiated by Petrov [99] and extensively studied by Polsky [131], [132], Vainikko [150] and others (see [72], [109] for further literature).

(iii) The generalized Ritz method. If $M=0, K=I$ and $L=A$, then $A_{0}$ is selfadjoint, $H_{0}=H_{A}$ and the GM method reduces to the method of Ritz studied by a number of authors and, in particular, by Mikhlin [86], [88]. For the extensive literature on this method, see [86]. If $K \neq I$, the generalized method of Ritz has been studied in [84], [102] and others. When $K \neq I$ and $L \neq A$ the method has been studied in [104]. When $K=A$ and $K$ is closed with $D(K)=D(L)$, then as was shown in [104], $L$ forms an acute angle with $K$ in the sense of Sobolevsky [143] and under the present conditions Theorem $2.2 \mathrm{C}$ was proved in [104] while under somewhat different conditions it was proved earlier by Sobolevsky [143].

(iv) The method of least squares. When $M=0$ and $L=A=K$, the GM method reduces to the method of least squares which has been studied by a number of authors (see [72], [86], [152] for references).

Advantages of the GM method. It is obvious that the first positive feature of the GM method is that it presents a unified approach to a number of special methods and that it is applicable to a more general class of equations than any of its special cases. Another of its advantages is that the wide freedom in the choice of $A$ and $K$ makes the GM method computationally more flexible and useful. When applied to differential equations it will give a better character of convergence than the method of Galerkin. For applications to partial differential equations see $\$ 2.4$.

Applications to ordinary differential equations $(O D E)$. We indicate here the type of problems to which the above results apply. Our simple example of a general third order linear ODE will also illustrate some of the points mentioned above. 
We discuss the approximation-solvability of the problem

$$
-\left(p_{3}(x) u^{\prime \prime}\right)^{\prime}+\sum_{i=0}^{2} p_{i}(x) u^{(i)}=f(x), \quad u(0)=u^{\prime}(0)=u^{\prime}(1)=0
$$

where $f \in L_{2} \equiv L_{2}(0,1), p_{i} \in C^{0}(0,1), p_{3} \in C^{1}(0,1)$ with $p_{3}(x) \geqq m_{0}>0$, and $C^{j}(0,1)$ denotes the set of all real-valued $v \in L_{2}$ which are $j$ times continuously differentiable on $[0,1]$. Let $C_{0}^{3}=\left\{u \in C^{3}(0,1) \mid u(0)=u^{\prime}(0)=\right.$ $\left.u^{\prime}(1)=0\right\}$.

To study equation (2.2-13) we take for $A$ the simple operator $A u=-u^{\prime \prime \prime}$ with $D(A)=C_{0}^{3}$ which is dense in $L_{2}$. If we take $K u=u^{\prime}$ with $D(K)=$ $\left\{u \in C^{1}(0,1) \mid u(0)=0\right\}$, then it is easy to show that the operator $A$ is $K$-p.d. and $K$-symmetric. In this case $H_{0}$ is the completion of $D(A)$ in the metric

$$
[u, v]=(A u, K v)=\int_{0}^{1} u^{\prime \prime} v^{\prime \prime} d x, \quad|u|=\sqrt{[u, u]} .
$$

The map $A$ has a unique $K$-p.d. and $K$-symmetric extension $A_{0}$ with $D\left(A_{0}\right) \subset H_{0}$ such that $A_{0}: D\left(A_{0}\right) \rightarrow H$ is bijective. It should be added that $\mathscr{W}_{2}^{2} \subset H_{0} \subset W_{2}^{2}$, where $W_{2}^{m}$ is the Sobolev space of all $u \in L_{2}$ such that $u$ and its derivatives up to order $m-1$ are absolutely continuous on $[0,1]$ and $u^{(m)} \in L_{2}$ and where $\stackrel{\circ}{W}_{2}^{m}=\left\{u \in W_{2}^{m} \mid u(0)=u(1)=\cdots=u^{(m-1)}(0)=\right.$ $\left.u^{(m-1)}(1)=0\right\}$. We denote the inner product and the norm in $W_{2}^{m}$ by $(\cdot, \cdot)_{m}$ and $\|\cdot\|_{m}$.

Problem 1. Suppose $L$ is defined by the left-hand side of (2.2-13) with $D(L)=D(A)$. Assume additionally that $p_{i}(x)$ satisfy further conditions such that $L$ is $K$-p.d. but not necessarily $K$-symmetric. If $\left\{\phi_{i}\right\} \subset D(L)$ is linearly independent and complete in $H_{0}$, then by Theorem $2.2 \mathrm{C}$ (with $M=0$ ) the GM method is applicable to equation (2.2-13) and the generalized solution $x_{0} \in H_{0}$ obtained by this method actually lies in $D\left(L_{0}\right)$, where $L_{0}$ is the solvable $K$-p.d. extension of $L$ given by Theorem 2.2A.

Problem 2. If we do not assume any additional conditions on $p_{i}(x)$, then we set

$$
L u=-\left(p_{3}(x) u^{\prime \prime}\right)^{\prime} \quad \text { for } u \in D(L)=D(A) \text { and } \quad M u=\sum_{i=0}^{2} p_{i} u^{(i)}
$$

and apply Theorem $2.2 \mathrm{C}$ to $L u+M u=f$. Integration by parts shows that $(L u, K u) \geqq m_{0}|u|^{2}$ for $u \in D(A)$, i.e., $L$ is $K$-p.d. It is easy to see that $m(u, v)=(M u, K v)$ is a bounded bilinear form on $H_{0}$ and that $|m(u, u)| \leqq$ $\gamma\|u\|_{2}\|u\|_{1}$. Hence, by the Sobolev imbedding theorem, the form $m(u, v)$ is completely continuous and therefore there exists a compact operator $C_{0}$ on $W_{2}^{2}$ such that $\left(C_{0} u, v\right)_{2}=m(u, v)$, for all $u$ and $v$ in $W_{2}^{2}$. Hence $A_{0}^{-1} M$ is compact on $H_{0}$ since $A_{0}^{-1} M=C_{0}$ on $H_{0}$. We can also prove the 
compactness of $A_{0}^{-1} M$ by using the Green's function for $A_{0}$ as it was done in [84]. Consequently, by Theorem $2.2 \mathrm{C}$, the GM method when applied to equation (2.2-13) converges in the space $H_{0}$. The generalized solution $u_{0} \in H_{0}$ need not lie in $D(L)$ and, in general, the residual error does not converge to 0 .

Problem 3. In this case we let $L$ and $M$ be as in Problem 2 but choose $K=A_{0}$ so that the metric on $D\left(A_{0}\right)$ is given by $|u|=\left\|A_{0} u\right\|=\left\|u^{\prime \prime \prime}\right\|$ where the derivatives are taken in generalized sense and with this metric $D\left(A_{0}\right)$ is denoted by $H_{0}$. It is not hard to show that $W_{2}^{3} \subset H_{0} \subset W_{2}^{3}$. Now the operator $L: H_{0} \rightarrow L_{2}$ is bounded but, unless further conditions are imposed on $p_{3}(x), L$ need not be $K$-p.d. for this choice of $K$. However, since, for $u \in D(L)=D\left(A_{0}\right)$,

$$
(L u, K u)=\int_{0}^{1} p_{3}\left(u^{\prime \prime \prime}\right)^{2}+\int_{0}^{1} p_{3}^{\prime}(x) u^{\prime \prime} u^{\prime \prime \prime} d x \geqq m_{0}|u|^{2}+b(u, u),
$$

where $b(u, v)$ is the bilinear form on $W_{2}^{3}$ given by $b(u, v)=\int_{0}^{1} p_{3}^{\prime} u^{\prime \prime} v^{\prime \prime \prime} d x$, the same arguments as those used in Problem 2 show that there exists a compact operator $C_{0}$ on $H_{0}$ such that $b(u, v)=\left[C_{0} u, v\right]$ for $u, v \in H_{0}$. Consequently, $L$ satisfies condition (a) of Theorem 2.2D. Similar arguments show that $M$ is $H_{0}$-compact. Hence, by Theorem 2.2E, $L+M: H_{0} \rightarrow$ $L_{2}$ is an $A$-proper mapping with respect to $\Gamma^{0}$ determined by $\left\{\phi_{i}\right\} \subset H_{0}$ and $\left\{K \phi_{i}\right\} \subset L_{2}$. Consequently, if $L+M$ is one-to-one, then Theorem 2.2E implies the unique approximation-solvability of equations (2.2-13) with respect to $\Gamma^{0}$. Moreover, the solution $u_{0}$ obtained by the GM method (2.2-9) as the limit of solutions $u_{n} \in X_{n}$ of equations (2.2-9) lies in $D(L)$ and $\left\|L u_{n}+M u_{n}-f\right\| \rightarrow 0$ as $n \rightarrow \infty$.

2.3. Generalized Fredholm alternative. Recall (see [64]) that $T \in L(X, Y)$ is said to be Fredholm if its range $R(T)$ is closed, and if the dimension $\alpha(T)$ of its null space $N(T) \subset X$ and the codimension $\beta(T)$ of its range are finite. If $T$ is Fredholm, then its index is given by $i(T)=\alpha(T)-\beta(T)$. In what follows we let $N(T)^{\perp}=\left\{u \in X^{*} \mid(u, x)=0\right.$ for $\left.x \in N(T)\right\}$ and $N\left(T^{*}\right)^{\perp}=\left\{y \in Y \mid(w, y)=0\right.$ for $\left.w \in N\left(T^{*}\right)\right\}$.

If $C \in L(X, X)$ is compact, then the classical Fredholm alternative asserts that: "Either equation (2.1-6) is uniquely solvable for each $y \in Y$ or $N(I-C) \neq\{0\}$ and then $\alpha(I-T)=\beta(I-C)=\operatorname{dim} N\left((I-C)^{*}\right)<\infty$ and equation (2.1-6) is solvable if and only if $f \in N\left((I-C)^{*}\right)^{\perp}$." As is well known, the above fact plays an essential role in the solvability of linear integral and differential equations.

Assuming that the pair $(X, Y)$ of Banach spaces has a projectionally complete scheme $\Gamma=\left\{X_{n}, P_{n} ; Y_{n}, Q_{n}\right\}$, the writer obtained in [113], [124] a generalization of the above alternative to $A$-proper mappings which we state in the following form. 
THEOREM 2.3A. Suppose $T \in L(X, Y)$ is A-proper. Then either equation (2.1) (i.e., $T x=y$ ) is uniquely approximation-solvable for each $y$ in $Y$ or $N(T) \neq\{0\}$ and in the latter case $T$ is Fredholm with $i(T) \geqq 0$.

It should be noted that the proof of Theorem 2.3A given in [124] is based on Theorem 2.1A and does not utilize the adjoint $T^{*}$ of $T$. If one assumes additionally that the adjoint scheme $\Gamma^{*}=\left\{Y_{n}^{\prime}, Q_{n}^{*} ; X_{n}^{\prime}, P_{n}^{*}\right\}$ is projectionally complete for the pair $\left(Y^{*}, X^{*}\right)$ where $Y_{n}^{\prime}=R\left(Q_{n}^{*}\right)$, and $X_{n}^{\prime}=R\left(P_{n}^{*}\right)$ (which would be the case if $X$ and $Y$ had shrinking Schauder bases), then it makes sense to talk about the $A$-properness of $T^{*}: Y^{*} \rightarrow X^{*}$ with respect to $\Gamma^{*}$. However, the following example from [116] shows that the adjoint $T^{*}$ of an $A$-proper mapping $T$ need not be $A$-proper with respect to $\Gamma^{*}$.

EXAMPLE 2.3-1. Suppose $Y=X=l_{2},\left\{\phi_{i}\right\}$ is an orthonormal basis in $l_{2}, X_{n}=\left[\phi_{1}, \cdots, \phi_{n}\right]$ for each $n$ and $P_{n}$ is an orthogonal projection of $l_{2}$ onto $X_{n}$. If $T \in L\left(l_{2}, l_{2}\right)$ is given by

$$
T \phi_{1}=0, \quad T \phi_{2}=0, \quad T \phi_{3}=\phi_{2}, \cdots, T \phi_{i}=\phi_{i-1} \quad \text { for } i \geqq 3,
$$

then $T$ is $A$-proper with respect to $\Gamma_{0}=\left\{X_{n}, P_{n} ; X_{n}, P_{n}\right\}$ but $T^{*}: l_{2} \rightarrow l_{2}$ is not $A$-proper with respect to $\Gamma_{0}^{*}=\Gamma_{0}$. Note that $N(T)=\left[\phi_{1}, \phi_{2}\right], N\left(T^{*}\right)=$ $\left[\phi_{1}\right]$ and so $i(T)=\alpha_{T}-\beta_{T}=1$.

The following result established by the writer in [113], [124] by using Theorems 2.1A and 2.1C supplements Theorem 2.3A and provides a complete generalization (partially in constructive form) of the classical Fredholm alternative.

TheOREM 2.3B. Let $T \in L(X, Y)$ and suppose that $\Gamma$ and $\Gamma^{*}$ are projectionally complete for $(X, Y)$ and $\left(Y^{*}, X^{*}\right)$ respectively. Then:

(a) $T$ is one-to-one and A-proper with respect to $\Gamma$ if and only if $T^{*}$ is one-to-one and A-proper with respect to $\Gamma^{*}$.

(b) Suppose $T$ is A-proper. Then either equation (2.1) is uniquely approximation-solvable for each $y$ in $Y$, in which case the adjoint equation

$$
T^{*}(u)=w \quad\left(u \in Y^{*}, w \in X^{*}\right)
$$

is also uniquely approximation-solvable with respect to $\Gamma^{*}$ for each $w$ in $X^{*}$, or $N(T) \neq\{0\}$ and then $i(T) \geqq 0$. Moreover, $i(T)=0$ if and only if $T^{*}$ is $A$-proper with respect to $\Gamma^{*}$.

REMARK 2.3-1. (i) For the case when $X$ and $Y$ are reflexive spaces Theorem 2.3B was proved by the author in [113]. In their present form Theorems 2.3A and 2.3B were obtained in [124] although they had been proved earlier in the writer's course on numerical functional analysis at Rutgers in 1969/70. 
(ii) If $Y=X, \Gamma=\Gamma_{0}=\left\{X_{n}, P_{n} ; X_{n}, P_{n}\right\}$ and $T=I-C$ with $C$ compact, then $T$ is $A$-proper and $T^{*}$ is also $A$-proper with respect to $\Gamma_{0}^{*}=$ $\left\{X_{n}^{\prime}, P_{n}^{*} ; X_{n}^{\prime}, P_{n}^{\prime}\right\}$ since $C^{*}$ is compact. Hence the classical Fredholm alternative (see [64]) follows as a special case from part (b) of Theorem 2.3B for the class of spaces considered in this paper.

(iii) If $Y=X, \Gamma=\Gamma_{0}$ with $\left\|P_{n}\right\|=1$ and $T=I-S-C$ with $\|S\|<1$, then $T$ is $A$-proper and so is $T^{*}=I^{*}-S^{*}-C^{*}$ with respect to $\Gamma_{0}^{*}$ since $\left\|S^{*}\right\|<1$ and $C^{*}$ is compact. Hence part (b) of Theorem $2.3 \mathrm{~B}$ is valid for $T=I-S-C$ with $i(T)=0$.

(iv) Suppose $Y=X, X$ is reflexive, $X$ and $X^{*}$ are strictly convex, and $\Gamma=\Gamma_{0}$ with $\left\|P_{n}\right\|=1$. If $T=L+C \in L(X, X)$ with $C$ compact and $(T x, J x)$ $\geqq c\|x\|^{2}$ for all $x \in X$ and some $c>0$, where $J$ is the normalized duality map of $X$ into $X^{*}$, then $T$ is $A$-proper with respect to $\Gamma_{0}$ and $T^{*}=L^{*}+$ $C^{*}: X^{*} \rightarrow X^{*}$ is also $A$-proper with respect to $\Gamma_{0}^{*}$. Hence part (b) of Theorem 2.3B is valid for $T=L+C$ with $i(T)=0$. If $X=H, H$ a separable Hilbert space, the above alternative was proven by Hildebrandt and Wienholtz [55].

(v) The Fredholm alternative for $A$-proper mappings defined in terms of more general schemes (the so-called discrete convergence of Stummel [145]) has been recently obtained by Grigorieff [51]. The results in [51] also extend the alternative of the writer obtained in [124].

It was mentioned before that if $T$ is $A$-proper and $C$ is compact, then $T+C$ is also $A$-proper. In applications it is of interest to know (just as in the Fredholm theory) if the map remains $A$-proper when perturbed by another map with a small norm. A partial answer is given by the following theorem (see [124]).

THEOREM 2.3C. If $T \in L(X, Y)$ is A-proper with $i(T)=0$, then there exists a constant $\gamma>0$ such that for each $B \in L(X, Y)$ with $\|B\|<\gamma$ the map $T+B$ is also A-proper.

It is not known to the writer if Theorem $2.3 \mathrm{C}$ remains valid when $i(T)>0$.

2.4. Dirichlet boundary value problem for elliptic equations. In this section we show how the notion of an $A$-proper mapping enters naturally into the problem of the solvability of boundary value problems (BVP) for elliptic partial differential equations. We illustrate this with the discussion of the approximation-solvability of the Dirichlet BVP studied by Vishik, Browder and Gårding (see [1]).

Let $Q$ be a bounded domain in $R^{n}$ with smooth boundary so that the Sobolev imbedding theorem holds on $Q$. For fixed $p \in(1, \infty)$ let $L_{p} \equiv$ $L_{p}(Q)$ denote the real Banach space of functions $u(x)$ on $Q$ with norm $\|u\|_{p}$. If $\alpha=\left(\alpha_{1}, \cdots, \alpha_{n}\right)$ is a multi-index of nonnegative integers we 
denote by $D^{\alpha}=\partial^{\alpha_{1}} / \partial x_{1}^{\alpha_{1}} \cdots \partial^{\alpha_{n}} / \partial x_{n}^{\alpha_{n}}$ a differential operator of order $|\alpha|=\alpha_{1}+\cdots+\alpha_{n}$. If $m$ is a nonnegative integer, $W_{p}^{m} \equiv W_{p}^{m}(Q)$ denotes the real Sobolev space of all $u \in L_{p}$ whose generalized derivatives $D^{\alpha} u,|\alpha| \leqq m$, also lie in $L_{p} . W_{p}^{m}$ is a separable uniformly convex Banach space with respect to the norm $\|u\|_{m, p}=\left\{\sum_{|\alpha| \leqq m}\left\|D^{\alpha} u\right\|_{p}^{p}\right\}^{p^{-1}}$. In case $p=2$, we get the Hilbert space $W_{2}^{m}$ with the corresponding inner product $(\cdot, \cdot)_{m}$. Let $C_{0}^{m}(Q)$ be the family of $m$ times continuously differentiable functions with compact support in $Q$ considered as a subset of $W_{p}^{m}$ and let $\stackrel{\circ}{W}_{p}^{m}$ be the completion in $W_{p}^{m}$ of $C_{0}^{m}(Q)$. Finally, let $\langle u, v\rangle=\int_{Q} u v d x$ denote the natural pairing between $u$ in $L_{p}$ and $v$ in $L_{a}$ with $q=p(p-1)^{-1}$.

We first consider the Dirichlet BVP of the form

$$
\mathscr{T}(u) \equiv \mathscr{L}(u)+\mathscr{M}(u)=f(x),\left.\quad D^{\omega} u\right|_{\partial Q}=0 \text { for }|\omega| \leqq m-1,
$$

where $f \in L_{2}$ and $\mathscr{L}(u)$ and $\mathscr{M}(u)$ are formal operators given by

$$
\begin{gathered}
\mathscr{L}(u)=\sum_{|\alpha|,|\beta| \leqq m}(-1)^{|\alpha|} D^{\alpha}\left(a_{\alpha \beta}(x) D^{\beta} u\right), \\
\mathscr{M}(u)=\sum_{|\beta| \leqq m-1,|\gamma| \leqq m}(-1)^{|\beta|} D^{\beta}\left(b_{\beta \gamma}(x) D^{\gamma} u\right)
\end{gathered}
$$

with $a_{\alpha \beta} \in L^{\infty}(\bar{Q})$ for $|\alpha| \leqq m$ and $|\beta| \leqq m$ and $a_{\alpha \beta} \in C(\bar{Q})$ for $|\alpha|=|\beta|=m$, and $b_{\beta \gamma} \in L^{\infty}(\bar{Q})$ for $|\beta| \leqq m-1$ and $|\gamma| \leqq m$.

The generalized Dirichlet $B V P$ for (2.4-1) consists in finding a function $u \in \mathscr{W}_{2}^{m}$, called the weak solution of (2.4-1), such that

$$
l(u, v)+m(u, v)=\langle f, v\rangle \text { for all } v \in \stackrel{\circ}{W}_{2}^{m},
$$

where $l(u, v)$ and $m(u, v)$ are the Dirichlet forms on $\stackrel{\circ}{W}_{2}^{m}$ associated with the formal differentiable operators $\mathscr{L}(u)$ and $\mathscr{H}(u)$ by

$$
\begin{aligned}
& l(u, v)=\sum_{|\alpha|,|\beta| \leqq m}\left\langle a_{\alpha \beta}(x) D^{\beta} u, D^{\alpha} v\right\rangle, \\
& m(u, v)=\sum_{|\beta| \leqq m-1,|\gamma| \leqq m}\left\langle b_{\beta \gamma}(x) D^{\gamma} u, D^{\beta} v\right\rangle .
\end{aligned}
$$

Our conditions on $a_{\alpha \beta}$ and $b_{\beta \gamma}$ imply that $l(u, v)$ and $m(u, v)$ are bounded bilinear forms on $\stackrel{\circ}{W}_{2}^{m}$ and hence can be written in the form

$$
l(u, v)=(L u, v)_{m}, \quad m(u, v)=(M u, v)_{m} \quad \text { for all } u, v \in \stackrel{\circ}{W}_{2}^{m},
$$

where $L, M: \stackrel{\circ}{W}^{m} \rightarrow \stackrel{\circ}{W}_{2}^{m}$ are bounded linear operators. If $w_{f} \in \stackrel{\circ}{W}_{2}^{m}$ is such that $\langle f, v\rangle=\left(w_{f}, v\right)_{m}$ for all $v$ in $\mathscr{W}_{2}^{m}$, then the equation

$$
L u+M u=w_{f} \quad\left(u \in \stackrel{\circ}{W}_{2}^{m}, w_{f} \in \stackrel{\circ}{W}_{2}^{m}\right)
$$

is equivalent to the conditions (2.4-4) for the weak solution of (2.4-1). 
Suppose that $\mathscr{L}(u)$ is strongly elliptic, i.e.,

$$
\sum_{|\alpha|,|\beta|=m} \alpha_{\alpha \beta}(x) \eta^{\alpha} \eta^{\beta} \geqq d|\eta|^{2 m} \text { for all } \eta \in R^{n} \text { and some } d>0 \text {. }
$$

Then, by Gårding inequality, there exist $a_{1}>0$ and $a_{0} \geqq 0$ such that

$$
(L u, u)_{m} \geqq a_{1}\|u\|_{m, 2}^{2}-a_{0}\|u\|_{0,2}^{2} \text { for all } u \in \stackrel{W}{W}_{2}^{m} .
$$

Since the imbedding of $\stackrel{\circ}{2}_{2}^{m}$ into $L_{2}$ is compact, it follows easily from (2.4-10) that $L$ is $A$-proper with respect to any given projectionally complete scheme $\Gamma_{0}=\left\{X_{n}, P_{n} ; X_{n}, P_{n}\right\}$ for $\left(\stackrel{\circ}{W}_{2}^{m}, W_{2}^{m}\right)$. The latter exist since $\mathscr{W}_{2}^{m}$ is a separable Hilbert space. Since, as is not hard to show,

$$
|m(u, u)| \leqq d_{0}\|u\|_{m, 2}\|u\|_{m-1,2} \text { for all } u \text { in } \dot{W}_{2}^{m} \text { and some } d_{0}>0,
$$

it follows that $M$ is compact. Hence $T=L+M: \stackrel{\circ}{W}_{2}^{m} \rightarrow \mathscr{W}_{2}^{m}$ is $A$-proper with respect to $\Gamma_{0}$ and consequently Theorem $2.1 \mathrm{~A}$ implies the validity of the following result.

THEOREM 2.4A. If $T=L+M: \stackrel{\circ}{W}_{2}^{m} \rightarrow \stackrel{\circ}{W}_{2}^{m}$ is one-to-one, then the generalized Dirichlet BVP for (2.4-1) is uniquely approximation-solvable with respect to $\Gamma_{0}$ for each $f$ in $L_{2}$. (In particular, (2.4-1) has a unique weak solution $u \in \mathscr{W}_{2}^{m}$ for each $f \in L_{2}$.)

REMARK 2.4-1. It should be underlined here that unlike the standard approach to the solvability of the generalized Dirichlet BVP (see [1], [5]) which employs the Lax-Milgram lemma and the Fredholm theorems with the latter depending on the use of adjoints of linear mappings, our proof of Theorem 2.4A which yields the constructive solvability of the generalized Dirichlet BVP for (2.4-1) does not utilize the notion of the adjoint of $T$. Consequently, similar arguments go over to nonlinear problems.

To discuss the case when $N(T) \neq\{0\}$ (i.e., the case when the generalized Dirichlet BVP (2.4-1) for $f=0$ has nontrivial weak solutions in $\grave{W}_{2}^{m}$ ) note first that $T^{*}=L^{*}+M^{*}: \stackrel{\circ}{W}_{2}^{m} \rightarrow \stackrel{\circ}{W}_{2}^{m}$ is also $A$-proper with respect to $\Gamma_{0}$ since $M^{*}$ is compact and $L^{*}$ satisfies the same inequality as $L$. Consequently, Theorem 2.3B yields the following result.

THEOREM 2.4B. If $N(T) \neq\{0\}$ and if $v_{1}, \cdots, v_{k}$ is a basis for $N\left(T^{*}\right) \subset$ $\stackrel{\circ}{W}_{2}^{m}$, then $k=\alpha(T)$ and the generalized Dirichlet BVP for (2.4-1) is solvable in $\stackrel{\circ}{W}_{2}^{m}$ for a given $f$ in $L_{2}$ if and only if $\left\langle f, v_{j}\right\rangle=0$ for $1 \leqq j \leqq k$.

Second order elliptic BVP in $\stackrel{\circ}{W}_{2}^{2}$. If the PDE is of the second order, then using the results on $A$-proper and $K$-p.d. mappings and the inequality of Sobolevsky [143] we obtain the unique approximation-solvability in $\mathscr{W}_{2}^{2}$ of the Dirichlet BVP for a general second order elliptic equation. 
Suppose $L$ is a strongly elliptic operator defined on $D(L)=\stackrel{\circ}{W}_{2}^{2}$ by

$$
L u=-\sum_{i, j=1}^{n} a_{i j}(x) \frac{\partial^{2} u}{\partial x_{i} \partial x_{j}}+\sum_{i=1}^{n} a_{i}(x) \frac{\partial u}{\partial x_{i}}+a_{0}(x) u
$$

and assume that $a_{i j}(x) \in C^{1}(Q), a_{i}(x) \in C(Q)$, and that $\partial Q$ is sufficiently smooth (see [103]). Our problem is to study the approximation-solvability of the equation

$$
L u=f \quad\left(u \in \stackrel{\circ}{W}_{2}^{2}, f \in L_{2}\right) .
$$

To use the theory of $K$-p.d. mappings let $T$ and $K$ be defined on $D(T)=\stackrel{\circ}{W}_{2}^{2}$ by

$$
T u=-\Delta u, \quad K u=-\Delta u+c u, \quad c>0 .
$$

It is known that $T$ is a selfadjoint positive definite map of $\mathscr{W}_{2}^{2}$ onto $L_{2}$. It was shown in [103] that for all sufficiently large $c>0$ the map $T$ is $K$-p.d. and $K$-symmetric and

$$
m_{1}(T u, K u) \leqq\|u\|_{2,2}^{2} \leqq m_{2}(T u, K u) \quad \text { for } u \in \stackrel{\circ}{W}_{2}^{2}
$$

with $m_{1}>0, m_{2}>0$. Consequently, $H_{0}=\stackrel{\circ}{W}_{2}^{2}$ where $H_{0}$ has the scalar product $[u, v]=(T u, K v)$. Let $\left\{\phi_{i}\right\} \subset H_{0}$ be linearly independent and complete in $H_{0}$. Then $\left\{K \phi_{i}\right\}$ is complete in $L_{2}$ and if $P_{n}: H_{0} \rightarrow X_{n}$ and $Q_{n}: L_{2} \rightarrow Y_{n}$ denote the orthogonal projections in $H_{0}$ and $L_{2}$ respectively with $X_{n}=$ $\left[\phi_{1}, \cdots, \phi_{n}\right]$ and $Y_{n}=\left[K \phi_{1}, \cdots, K \phi_{n}\right]$, then $\Gamma^{0}=\left\{X_{n}, P_{n} ; Y_{n}, Q_{n}\right\}$ is projectionally complete for $\left(H_{0}, L_{2}\right)$.

The projection method for equation (2.4-12) consists in finding $x_{n} \in X_{n}$ from the finite dimensional operator equation

$$
Q_{n} L x_{n}=Q_{n} f \quad\left(x_{n} \in X_{n}, Q_{n} f \in Y_{n}\right) .
$$

It follows from (2.4-14) that $T, K$ and $L$, considered as mappings from $H_{0}$ to $L_{2}$, are bounded. By the inequality of Sobolevsky [143] (see also [74]) there exist constants $\tau_{1}>0$ and $\tau_{2}>0$ such that

$$
(L u, K u) \geqq \tau_{1} \int_{Q_{i}, j=1} \sum^{n}\left(\frac{\partial^{2} u}{\partial x_{i} \partial x_{j}}\right)^{2} d x-\tau_{2}\|u\|_{1,2}^{2} \quad \text { for } u \in H_{0} .
$$

It is easy to see that this inequality can be put in the form

$$
(L u, K u) \geqq \gamma_{1}|u|^{2}-\tau_{2}\left[C_{0} u, u\right] \text { for } u \in H_{0}, \gamma_{1}>0,
$$

where $C_{0}$ is the compact map determined by the bilinear form $(u, v)_{1}$ on $H_{0}$ which is compact since the injection of $\stackrel{\circ}{W}_{2}^{2} \rightarrow W_{2}^{1}$ is compact. Hence, by Lemma $2.1 \mathrm{H}, L: H_{0} \rightarrow L_{2}$ is $A$-proper with respect to $\Gamma^{0}$.

The above discussion implies the validity of the following new constructive theorem for the Dirichlet BVP for the second order strongly elliptic operator in $\stackrel{\circ}{W}_{2}^{2}$. 
THEOREM 2.4C. Let $L$ be the strongly elliptic operator defined on $\stackrel{\circ}{W}_{2}^{2}$ by (2.4-11) and let $\Gamma^{0}$ be the projectionally complete scheme for the pair $\left(H_{0}, L_{2}\right)$ determined by $\left\{\phi_{i}\right\} \subset \mathscr{W}_{2}^{2}$ and $\left\{K \phi_{i}\right\} \subset L_{2}$, where $K$ is defined by (2.4-13) and satisfies (2.4-14).

If $L$ is one-to-one, then equation (2.4-12) is uniquely approximationsolvable in $H_{0}=\stackrel{\circ}{W}_{2}^{2}$ with respect to $\Gamma^{0}$ for each $f \in L_{2}$.

REMARK 2.4-2. (i) Theorem 2.4C strengthens the corresponding result of Ladyzenskaya [74] who established the approximation solvability of equation (2.4-12) under the assumption that equation (2.4-12) is uniquely solvable for each $f \in L_{2}$ and that $L$ satisfies the inequality

$$
-(L u, u) \geqq c\|u\|_{2,1}^{2} \text { for all } u \in \mathscr{W}_{2}^{2} \text { and some } c>0 .
$$

(ii) Theorem $2.4 \mathrm{C}$ provides a new proof for the existence of solutions in $\stackrel{\circ}{W}_{2}^{2}$ of equation (2.4-12). It does not use the classical Fredholm alternative which was used in all other studies known to the author concerning the solvability of (2.4-12) in $\mathscr{W}_{2}^{2}$.

2.5. Stability of projective methods and A-properness. The study of the stability of projective methods was initiated by Mikhlin [88] who concentrated his attention on the Ritz method (see [88]) while the stability of the Galerkin and the Galerkin-Petrov methods was considered in [162] and later more completely in [150] and others (see [33], [88]). All results for $A$-proper maps presented here are new.

In this section we indicate the relation of the notion of the $A$-properness of $T$ with respect to $\Gamma=\left\{X_{n}, P_{n} ; Y_{n}, Q_{n}\right\}$ to the notion of the stability of the method

$$
T_{n}\left(x_{n}\right)=Q_{n} f \quad\left(x_{n} \in X_{n}, T_{n}=\left.Q_{n} T\right|_{X_{n}}, n \geqq n_{0}\right)
$$

as defined by Mikhlin [88] with a slight variant due to [150] and [33] (see also [72]).

For each fixed $n\left(\geqq n_{0}\right)$, along with equation (2.5-1) we consider the perturbed equation

$$
\left(T_{n}+F_{n}\right)\left(y_{n}\right)=Q_{n} f+h_{n} \quad\left(y_{n} \in X_{n}, h_{n} \in Y_{n}\right),
$$

where $F_{n}: X_{n} \rightarrow Y_{n}$ is a linear operator perturbation of $T_{n}$ and $h_{n}$ is a perturbation of $Q_{n} f$.

Definition 2.5A. Suppose that equation (2.5-1) is uniquely solvable for each $n \geqq n_{0}$ and each $f \in Y$. Then the projection method (2.5-1) is said to be stable from space $X$ to $Y$ if there exist nonnegative constants $p, q, r$ independent of $n$ and $f$ such that for $\left\|F_{n}\right\| \leqq r$ and arbitrary $h_{n}$ in $Y_{n}$ the perturbed equation (2.5-2) has a unique solution $y_{n} \in X_{n}$ for $n \geqq n_{0}$ 
and the following inequality holds:

$$
\left\|x_{n}-y_{n}\right\| \leqq p\left\|x_{n}\right\|\left\|F_{n}\right\|+q\left\|h_{n}\right\| \quad\left(n \geqq n_{0}\right) .
$$

THEOREM 2.5B. Suppose that $T \in L(X, Y)$ is surjective. Then the projective method (2.5-1) is stable in the sense of Definition 2.5A if and only if $T$ is one-to-one and A-proper with respect to $\Gamma$.

Proof. Suppose $T$ is one-to-one and $A$-proper. Then, by Theorem 2.1A, equation (2.5-1) is uniquely solvable for each $n \geqq n_{0}$ and each $f \in Y$ and so it makes sense to talk about the stability of (2.5-1) as given by Definition 2.5A. Moreover, there exists $c>0$ such that $\left\|T_{n}(x)\right\| \geqq c\|x\|$ for $x \in X_{n}$ and $n \geqq n_{0}$. Let $r$ be any fixed number in $[0, c)$ and let $\left\|F_{n}\right\| \leqq r$. Then for all $x$ in $X_{n}$ and $n \geqq n_{0}$ we have $\left\|\left(T_{n}+F_{n}\right) x\right\| \geqq(c-r)\|x\|$. Hence for each fixed $n \geqq n_{0}$ and any $h_{n} \in Y_{n}$, equation (2.5-2) has a unique solution $y_{n} \in X_{n}$. The above discussion implies the equality

$$
x_{n}-y_{n}=\left(T_{n}+F_{n}\right)^{-1}\left(F_{n}\left(x_{n}\right)-h_{n}\right)
$$

from which (2.5-3) follows with $p=q=(c-r)^{-1}$.

To prove the converse, suppose that (2.5-1) is stable in the sense of Definition 2.5A. Take $F_{n}=0$ and for arbitrary $f \in Y$ and $h_{n} \in Y_{n}$ let $x_{n}$ and $y_{n}$ be the solutions for $n \geqq n_{0}$ of the corresponding equations (2.5-1) and (2.5-2). Hence it follows from (2.5-3) that $\left\|x_{n}-y_{n}\right\| \leqq q\left\|h_{n}\right\|=$ $\left\|T_{n}\left(x_{n}-y_{n}\right)\right\|$ and consequently $\left\|T_{n}(x)\right\| \geqq q^{-1}\|x\|$ for all $x \in X_{n}$ and each $n \geqq n_{0}$. In view of this and the surjectivity of $T$, it follows from Theorem 2.1B that $T$ is one-to-one and $A$-proper. Q.E.D.

Note that if $X$ is reflexive and $Q_{n} Q_{m}=Q_{n}$ for $m \geqq n$ or $Q_{n}^{*} u \rightarrow u$ in $Y^{*}$ for each $u$ in $Y^{*}$, then the hypothesis in Theorem $2.5 \mathrm{~B}$ that $T$ is surjective can be omitted.

Theorem 2.5B forms a basis for the study of stability of the numerical realization of the approximation methods of the Galerkin type when the latter are applied to the solvability of equation (2.1) involving $A$-proper mappings.

GALERKIN METHOD. We illustrate the above assertion by discussing the stability of the Galerkin method when the latter is applied to the equation

$$
T x=f \quad(f \in H, T \in L(H, H)),
$$

where $H$ is a separable real Hilbert space with a linearly independent and complete system $\left\{\phi_{i}\right\} \subset H$ and where $T$ is one-to-one $A$-proper with respect to $\Gamma_{0}=\left\{X_{n}, P_{n} ; X_{n}, P_{n}\right\}$ with $P_{n}$ the orthogonal projection of $H$ onto $X_{n}=\left[\phi_{1}, \cdots, \phi_{n}\right]$ for each $n \geqq 1$.

If the approximate solution $x_{n} \in X_{n}$ is taken in the form

$$
x_{n}=\sum_{i=1}^{n} a_{i}^{n} \phi_{i}
$$


then in the Galerkin method the unknowns $a_{i}^{n}$ are defined by the algebraic system

$$
\sum_{i=1}^{n}\left(T \phi_{i}, \phi_{j}\right) a_{i}^{n}=\left(f, \phi_{j}\right) \quad(1 \leqq j \leqq n)
$$

which can be written in the form

$$
G_{n} a^{(n)}=f^{(n)},
$$

where $a^{(n)}=\left(a_{1}^{n}, \cdots, a_{n}^{n}\right)^{T}, f^{(n)}=\left(\left(f, \phi_{1}\right), \cdots,\left(f, \phi_{n}\right)\right)^{T}, G_{n}=\left(\left(T \phi_{i}, \phi_{j}\right)\right)$ for $1 \leqq i, j \leqq n$. Let $\gamma_{i j}$ denote the errors arising in the computation of $\left(T \phi_{i}, \phi_{j}\right)$, let $\Gamma_{n}=\left(\gamma_{i j}\right)$ be the error matrix, and let $\delta^{(n)}$ be the corresponding error in $f^{(n)}$. Then instead of the exact Galerkin process (2.5-6) we solve the "nonexact" process

$$
\left(G_{n}+\Gamma_{n}\right) b^{(n)}=f^{(n)}+\delta^{(n)},
$$

and obtain the "nonexact" solution

$$
y_{n}=\sum_{i=1}^{n} b_{i}^{n} \phi_{i} \text {. }
$$

Following Mikhlin [88] (with a variant due to Vainikko [150]) we say that the Galerkin method is stable if there exist nonnegative constants $r, p, q$ independent of $n$ and of $f$ such that for $\left\|\Gamma_{n}\right\| \leqq r$ and arbitrary $\delta^{(n)} \in R^{n}$ the perturbed equation (2.5-8) has a unique solution $b^{(n)} \in R^{n}$ for $n \geqq n_{0}$ and

$$
\left\|a^{(n)}-b^{(n)}\right\| \leqq p\left\|x_{0}\right\|\left\|\Gamma_{n}\right\|+q\left\|\delta^{(n)}\right\|
$$

where $x_{0}$ is the unique solution of equation (2.5-4) which exists by Theorem 2.1A. By the norms of the vectors and matrices we mean their norms as elements and operators in the space $R^{n}$.

Now, as was mentioned before, equation (2.5-6) may be written in $X_{n}$ as

$$
P_{n} T x_{n}=P_{n} f \quad\left(x_{n} \in X_{n}, P_{n} f \in X_{n}\right) .
$$

To use Theorem 2.5B we have to write equation $(2.5-8)$ as an operator equation in $X_{n}$. For this purpose we use the approach of [72]. Let $S_{n}: H \rightarrow$ $R^{n}$ be the linear map defined by $S_{n}(x)=\left(\left(x, \phi_{1}\right), \cdots,\left(x, \phi_{n}\right)\right)^{T}$ for each $x$ in $H$ and let $S_{n}$ be the restriction of $S_{n}$ to $X_{n}$. It follows that $\left\|S_{n}\right\|=$ $\left\|S_{n}\right\|, S_{n}^{-1}$ exists and $S_{n}^{-1} S_{n}=P_{n}$. Moreover, if $u_{n}=\sum_{i=1}^{n} c_{i}^{n} \phi_{i}$ is any element in $X_{n}$, then $S_{n}\left(u_{n}\right)=R_{n} c^{(n)}$, where $R_{n}$ is the Gramm matrix given by $R_{n}=\left(\left(\phi_{i}, \phi_{j}\right)\right)$ for $1 \leqq i, j \leqq n$ and each $n \geqq 1$. Since $c^{(n)}=R_{n}^{-1} S_{n}\left(u_{n}\right)$, the above discussion implies that equation (2.5-8) can be written in $X_{n}$ as

$$
P_{n} T y_{n}+F_{n} y_{n}=P_{n} f+h_{n},
$$

where $h_{n}=S_{n}^{-1} \delta^{(n)} \in X_{n}$ and $F_{n}=S_{n}^{-1} \Gamma_{n} R_{n}^{-1} S_{n}: X_{n} \rightarrow X_{n}$. 
Now, since $R_{n}$ is a positive symmetric matrix its eigenvalues $\lambda_{i}^{n}$ are positive and we order them by $0<\lambda_{1}^{n} \leqq \cdots \leqq \lambda_{n}^{n}$. Following the accepted terminology we say that the system $\left\{\phi_{i}\right\}$ is strongly minimal if there exists a constant $\lambda_{0}>0$ such that $\inf _{n} \lambda_{1}^{n}=\lim _{n} \lambda_{1}^{n} \geqq \lambda_{0}$. Of course an orthogonal system in $H$ or even "almost orthogonal system $\left\{\phi_{i}\right\}$ in $H$ " is strongly minimal.

THEOREM 2.5C. If $T \in L(H, H)$ is one-to-one and A-proper with respect to $\Gamma_{0}$ and if $\left\{\phi_{i}\right\}$ is strongly minimal in $H$, then equation (2.5-4) is uniquely approximation-solvable and the Galerkin method (2.5-5)-(2.5-6) is stable.

Proof. It follows from the results in [72], [88] and the strong minimality of $\left\{\phi_{i}\right\}$ in $H$ that $\left\|S_{n}^{-1}\right\| \leqq \lambda_{0}^{-1 / 2}$ and $\left\|R_{n}^{-1} S_{n}\right\| \leqq \lambda_{0}^{-1 / 2}$ for each $n \geqq 1$. Hence

$$
\left\|F_{n}\right\| \leqq\left\|S_{n}^{-1}\right\|\left\|\Gamma_{n}\right\|\left\|R_{n}^{-1} S_{n}\right\| \leqq \lambda_{0}^{-1}\left\|\Gamma_{n}\right\| \text { and }\left\|h_{n}\right\| \leqq \lambda_{0}^{-1 / 2}\left\|\delta^{(n)}\right\|
$$

for each $n$. Now, since $T$ is one-to-one and $A$-proper with respect to $\Gamma_{0}$, there exists a constant $c>0$ and an integer $n_{0} \geqq 1$ such that $\left\|T_{n}(x)\right\| \geqq c\|x\|$ for all $x \in X_{n}$ and $n \geqq n_{0}$. Consequently, if we assume that $\left\|\Gamma_{n}\right\|<r=$ $\frac{1}{2}\left(c / \lambda_{0}\right)$, then $\left\|F_{n}\right\|<\frac{1}{2} c$ and so, by Theorem 2.5B, equation (2.5-12), or equivalently equation (2.5-8), has a unique solution $b^{(n)}$ for $n \geqq n_{0}$. For $x_{n}$ and $y_{n}$ given by (2.5-5) and (2.5-9) respectively we have the inequality $(2.5-3)$, which in our case reduces to

$$
\left\|x_{n}-y_{n}\right\| \leqq \frac{2}{c}\left\|x_{n}\right\|\left\|F_{n}\right\|+\frac{2}{c}\left\|h_{n}\right\| .
$$

Since $x_{n}$ is the solution of equation (2.5-1) and $x_{n} \rightarrow x_{0}$ in $H$, there exists a constant $\sigma>0$ independent of $n$ or $f$ such that $\left\|x_{n}\right\| \leqq \sigma\left\|x_{0}\right\|$. Now, since $\left(R_{n} e^{(n)}, e^{(n)}\right) \geqq \lambda_{0}\left\|e^{(n)}\right\|^{2}$ for all $e^{(n)}$ in $R_{n}$ and for each $n$ and

$$
\left\|x_{n}-y_{n}\right\|^{2}=\left(R_{n}\left(a^{(n)}-b^{(n)}\right), a^{(n)}-b^{(n)}\right),
$$

the inequality $(2.5-10)$ follows from the above discussion and the inequality (2.5-13) with $p=\sigma c^{-1} \lambda_{0}^{-1 / 2}$ and $q=2 c^{-1}$. Hence the Galerkin method (2.5-5)$(2.5-6)$ is stable.

REMARK 2.5-1. Since $T=I-C$ is $A$-proper when $C$ is compact, Theorem $2.5 \mathrm{C}$ implies the stability of the Galerkin method obtained in [162].

\section{Equations involving nonlinear $A$-proper mappings}

In this chapter we show that the approximation-solvability results obtained for linear equations admit extensions to equations

$$
T x=f \quad(x \in X, f \in Y)
$$


involving nonlinear $A$-proper mappings under suitable additional conditions on $T$. As before we associate with equations (3.1) nonlinear finite-dimensional approximate equations

$$
T_{n}(x)=Q_{n} f \quad\left(x \in X_{n}, T_{n}=\left.Q_{n} T\right|_{X_{n}}, Q_{n} f \in Y_{n}\right),
$$

where $\Gamma=\left\{X_{n}, P_{n} ; Y_{n}, Q_{n}\right\}$ is a given projectionally complete scheme for Banach spaces $(X, Y)$.

In $\$ 3.1$ we survey the approximation-solvability results for equation (3.1) involving $A$-proper maps which satisfy either a nonlinear analogue of an $a$-stability condition (3.1-1), $K$-coerciveness, or condition ( + ). Applications are made to special classes of equations. Various sufficient conditions for the $A$-properness of $T$ are given and a BVP for nonlinear elliptic equations is discussed. In $\$ 3.2$ we study the approximationsolvability of equations involving odd $A$-proper maps with applications to various special classes of mappings. $\$ 3.3$ treats the Fredholm alternative for asymptotically linear $A$-proper mappings with applications to nonlinear elliptic boundary value problems.

3.1. Equation with approximation-stable A-proper mapping. The following nonlinear analogue of Theorem 2.1A, which was proved by the author [112] characterizes nonlinear $A$-proper mappings if they are approximationstable ( $a$-stable), where a map $T: X \rightarrow Y$ is said to be $a$-stable (with respect to $\Gamma$ ) provided there exists a gauge function $c(r)$ and an integer $n_{0} \geqq 1$ such that for each $n \geqq n_{0}$

$$
\left\|T_{n}(x)-T_{n}(y)\right\|>c(\|x-y\|) \text { for } x, y \in X_{n} .
$$

TheOREM.3.1A. Suppose $T: X \rightarrow Y$ is fa-continuous and approximationstable. Then equation (3.1) is uniquely approximation-solvable for each $f$ in $Y$ if and only if $T$ is A-proper and one-to-one.

It is useful to note that the proof of Theorem 3.1A does not use the hypothesis that $P_{n}(x) \rightarrow x$ for $x \in X$. If the latter is used and $Q_{n} T P_{n} x \rightarrow T x$ for $x \in X$, then Theorem 3.1A remains valid without the condition that $T$ is one-to-one.

In view of the discussion in $\$ 1.1$, Theorem 3.1A implies the following corollary from which one deduces the surjectivity theorems for strongly monotone operators of Minty [89], Browder [19], Shinbrot [140], and Zarantonello [163] in case of complex monotone maps.

COROLlaRY 3.1B. Let $X$ be reflexive with a projectionally complete scheme $\Gamma_{1}$ for $\left(X, X^{*}\right)$. If $T: X \rightarrow X^{*}$ is strongly monotone and either continuous, demicontinuous, or weakly continuous, then equation (3.1) is uniquely approximation-solvable for each $f$ in $X^{*}$. 
As will be shown elsewhere, the importance of Theorem 3.1A lies in the fact that if (3.1-1) holds, then the method (3.2) is stable in a sense analogous to that used in $\$ 2.5$. Theorem 3.1A points to the importance of finding conditions which, assuming (3.1-1), would imply that $T$ is $A$ proper. To that end it was shown in [110] that if $X$ and $Y$ are reflexive, if $Q_{n} T P_{n} x \rightarrow T x$ for $x \in X$, and if $T$ satisfies (3.1-1), then equation (3.1) is uniquely approximation-solvable if and only if $T$ satisfies

Condition (c). If $\left\{x_{n_{j}} \mid x_{n_{j}} \in X_{n_{j}}\right\}$ is such that $x_{n_{j}} \rightarrow x$ in $X$ and $T_{n_{j}}\left(x_{n_{j}}\right) \rightarrow g$ for some $g$ in $Y$, then $T(x)=g$.

To see for what classes Condition (c) can be verified by direct arguments we consider the following (see [115], [119]).

Definition 3.1C. Let $K$ be a map of $X$ into $Y^{*}$ such that $K(0)=0$, $K x \neq 0$ for $x \neq 0$. A mapping $T: X \rightarrow Y$ is called strongly $K$-monotone if $(T x-T y, K(x-y)) \geqq c(\|x-y\|)\|K(x-y)\|$ for $x, y \in X$, where $c(r)$ is some gauge function.

Lemma 3.1D. Let $\Gamma$ be a scheme for $(X, Y)$ with $X$ reflexive and let $K: X \rightarrow Y^{*}$ be such that

$$
Q_{n}^{*} K x=K x \text { for all } x \in X_{n} \text { and each } n .
$$

If $T: X \rightarrow Y$ is a strongly $K$-monotone mapping, then $T$ satisfies Condition (c) if one of the following two conditions holds:

(a) $T$ is continuous everywhere and $K$ is weakly continuous at 0 .

(b) $T$ is demicontinuous and bounded and $K$ is strongly and weakly continuous with $R(K)$ dense in $Y^{*}$, and for each $t>0$ there is a $k_{t}>0$ such that $K(t x)=k_{t} K(x)$ for $x \in X$.

In view of Lemma 3.1D, an immediate consequence of Theorem 3.1A is the following corollary which contains the results of Browder-De Figueiredo [22] for strongly $J$-monotone operators when $Y=X$ and $K=J$ and Corollary 3.1B when $Y=X^{*}$ and $K=I$ (see also [105] with $K$ linear).

Corollary 3.1E. Suppose $T: X \rightarrow Y$ is strongly $K$-monotone and either (a) or (b) of Lemma 3.1D holds. Then equation (3.1) is uniquely approximation-solvable for each $f \in Y$.

Note that the most restrictive condition in Corollary $3.1 \mathrm{E}$ that $K$ be weakly continuous everywhere is always satisfied if $K$ is linear.

It was observed by Browder [13] that in some cases (as in Lemma 3.1D) the verification of Condition (c) by direct arguments would require a considerable strengthening of the hypotheses (e.g. that $K$ be weakly 
continuous). Instead of Condition (c) he proposed the assumption of the solvability hypothesis and with it proved in [13] the following

THEOREM 3.1F. Suppose $T: X \rightarrow Y$ is continuous and approximationstable (i.e. 3.1-1 holds). Then equation (3.1) is uniquely approximationsolvable for a given $f \in Y$ if and only if it is solvable.

In view of Theorems $3.1 \mathrm{~A}$ and $3.1 \mathrm{~F}$, one has the following characterization of the $A$-properness of $T$ analogous to Theorems $2.1 \mathrm{~A}-2.1 \mathrm{C}$ in the linear theory.

THEOREM 3.1G. If $T: X \rightarrow Y$ is continuous and approximation-stable, then the following assertions are equivalent:

(a) $T$ is A-proper and one-to-one,

(b) $R(T)=Y$,

(c) If $\left\{x_{n_{j}} \mid x_{n_{j}} \in X_{n_{j}}\right\}$ is bounded and $T_{n_{j}}\left(x_{n_{j}}\right) \rightarrow g$ for some $g$ in $Y$, then there exists $x \in X$ such that $T x=g$.

The practical usefulness of Theorem $3.1 \mathrm{G}$ stems from the fact that if we know (no matter how) that $T$ is surjective, then under condition (3.1-1) the map $T$ is one-to-one and $A$-proper and thus equation (3.1) is uniquely approximation-solvable for each $f \in Y$.

Using Browder's surjectivity Theorem 4 in [18] and Theorem 3.1G, one immediately deduces from Theorem $3.1 \mathrm{~A}$ a considerable strengthening of Corollary 3.1E.

CoROllarY 3.1H. Let $X$ and $Y$ be Banach spaces with $Y^{*}$ uniformly convex. Suppose $T: X \rightarrow Y$ is locally Lipschitzian and strongly $K$-monotone with $c(r)=r$ and $\|K x\|=\|x\|$ for all $x$ in $X$ and such that (3.1-2) holds. Then equation (3.1) is uniquely approximation-solvable for each $f \in Y$.

It will be seen below that one can obtain approximation-solvability results for equation (3.1) involving $A$-proper mappings $T$ without the assumption that $T$ is $a$-stable or strongly $K$-monotone. In view of this, it is important to find weaker conditions which would nevertheless imply the $A$-properness of $T: D \subseteq X \rightarrow Y$.

The first results in this direction for bounded continuous mappings $T$ acting from a separable reflexive space $X$ into $X^{*}$ were obtained by Pokhodjayev (see Condition 1 in [130]) and in somewhat more general form by Browder (see Condition (S) in [19]). In [119] the author extended the results of [19], [130] by modifying the condition (S) of Browder in such a way as to be applicable to mappings $T: D \subset X \rightarrow Y$ and to be more in consonance with the notion of the $A$-properness with respect to a given scheme 
$\Gamma$. Since the sum of two $A$-proper mappings need not be $A$-proper [116], Browder [19] restricted the class of mappings satisfying condition (S) to those satisfying condition $\left(\mathrm{S}_{+}\right)$and showed that if $T$ satisfies condition $\left(\mathrm{S}_{+}\right)$, then $T_{t}(x)=(1-t) T(x)-t T(-x)$ is $A$-proper for each $t \in[0,1]$. The latter result is important in various applications (see [16], [119]) of the theory of the generalized topological degree for $A$-proper mappings developed in Browder-Petryshyn [24], [25]. In [119] the author studied the relation of the $A$-properness of $T: D \subset X \rightarrow Y$ to the modified conditions $(\mathrm{S})$ and $\left(\mathrm{S}_{+}\right)$and, in particular, to mappings satisfying the generalized pseudo-monotonicity condition of Brezis [8]. The following result from [115], [119] will justify the preceding remarks and at the same time exhibit further classes of nonlinear mappings to which the theory of $A$ proper mappings is applicable.

Definition 3.1I. Let $X$ be a reflexive Banach space, $\Gamma=\left\{X_{n}, P_{n}\right.$; $\left.Y_{n}, Q_{n}\right\}$ a projectionally complete scheme for $(X, Y), K$ a mapping of $X$ into $Y^{*}, D$ an open convex subset of $X$, and $T: \bar{D} \rightarrow Y$ continuous. The mapping $T$ is said to satisfy the modified condition (S) (resp. modified condition $\left.\left(\mathrm{S}_{+}\right)\right)$if for any $\left\{x_{n_{j}} \mid x_{n_{j}} \in X_{n_{j}} \cap \bar{D}\right\}$ for which $x_{n_{j}} \rightarrow x_{0}$ in $X$ and $\left(T x_{n_{j}}-T y_{n_{j}}, K\left(x_{n_{j}}-y_{n_{j}}\right)\right) \rightarrow 0$ (resp. $\lim \sup _{n}\left(T x_{n_{j}}-T y_{n_{j}}, K\left(x_{n_{j}}-y_{n_{j}}\right)\right) \leqq$ 0) we have $x_{n_{j}} \rightarrow x_{0}$ in $X$ as $j \rightarrow \infty$, where $\left\{y_{n_{j}} \mid y_{n_{j}} \in X_{n_{j}} \cap \bar{D}\right\}$ is such that $y_{n_{j}} \rightarrow x_{0}$ as $j \rightarrow \infty$.

THEOREM 3.1J. Suppose the conditions of Definition 3.1I hold with the additional assumption that $K$ is weakly continuous at 0 and (3.1-2) holds. Let $C: \bar{D} \rightarrow Y$ be compact and $f(x)$ a functional on $X$ with $f(0)=0$. Then the mapping $T: \bar{D} \rightarrow Y$ is A-proper with respect to $\Gamma$ if any one of the following hypotheses holds:

(J1) $T$ satisfies the modified condition (S);

(J2) $X$ has property $(\mathrm{H})$ and there exists a continuous strictly increasing function $\psi$ of $R^{+}$into $R^{+}$with $\psi(r) \rightarrow \infty$ as $r \rightarrow \infty$ such that for each $n$

$$
(T x-T y, K(x-y)) \geqq(\psi(\|x\|)-\psi(\|y\|))(\|x\|-\|y\|) \text { for } x, y \in X_{n} \cap \bar{D} \text {. }
$$

(J3) $f$ is weakly upper semicontinuous at 0 and there exists a continuous increasing function $c(r)$ of $R^{+}$into $R^{+}$with $r \rightarrow 0$ as $c(r) \rightarrow 0$ such that $(T x-T y, K(x-y))+(C x-C y, K(x-y))+f(x-y) \geqq c(\|x-y\|)$ for $x, y \in$ $X_{n} \cap \bar{D}$.

REMARK 3.1-1. If we additionally assume that $D$ is symmetric about $0 \in D$ and $K$ is odd (i.e., $K(-x)=-K x$ for $x \in X$ ) and define

$$
H_{t}(x)=\frac{1}{1+t} T(x)-\frac{t}{1+t} T(-x)
$$


for $x \in \bar{D}$ and $t \in[0,1]$, then $H_{t}$ is $A$-proper for each $t \in[0,1]$ if $T$ satisfies the modified condition $\left(\mathbf{S}_{+}\right)$and, in particular, if either (J2) or (J3) holds. The last assertion also holds if

(J4) $T$ is of modified type (S), $f$ is weakly continuous at 0 and $(T x-T y$, $K(x-y))+(C x-C y, K(x-y))+f(x-y) \geqq 0$ for $x, y \in X_{n} \cap \bar{D}$ and $n \geqq 1$.

RemarK 3.1-2. Even when $D=X$ the hypotheses in Theorem 3.1J are required to hold only on the dense set $\bigcup X_{n}$ and not on the whole of $X$ as in [9], [130] for $Y=X^{*}$ and $K=I$. When $D=X, Y=X^{*}, K=I, T$ bounded and continuous and $f$ weakly upper semicontinuous on $X$, Theorem 3.1 J under condition (J3) was first proved in [130]. Independently of [119] and under stronger conditions, Theorem 3.1J under conditions (J2) and (J3) were also obtained in [44].

In what follows we say that $T: X \rightarrow Y$ is $K$-coercive if there exists a function $c(r)$ of $R^{+}$into $R^{1}$ with $c(r) \rightarrow \infty$ as $r \rightarrow \infty$ such that

$$
(T x, K x) \geqq c(\|x\|)\|K x\| \quad \text { for } x \in X .
$$

Our next result is the following special case from [115] which establishes the approximation-solvability of $K$-coercive $A$-proper mappings.

Theorem 3.1K. Let $(X, Y)$ be Banach spaces, $K$ a map of $X$ into $Y^{*}$ for which (3.1-2) holds and $T: X \rightarrow Y A$-proper and $K$-coercive. Let $M_{n}: X_{n} \rightarrow$ $Y_{n}$ be a linear isomorphism such that

$$
\left(M_{n}(x), Q_{n}^{*} K x\right)>0 \text { for } x \in X_{n} \text { with } x \neq 0 .
$$

Then equation (3.1) is feebly approximation-solvable for each $f$ in $Y$. Equation (3.1) is strongly approximation-solvable if it is uniquely solvable for a given $f$.

The proof of Theorem $3.1 \mathrm{~K}$ is based on the properties of the Brouwer degree for mappings of oriented finite dimensional spaces of the same dimension (see [28]) and the following lemma.

Lemma 3.1L. If $D$ is a bounded open subset of $X$ and $T$ an A-proper map of $\bar{D}$ into $Y$ such that for some $g \in Y$

$$
T x-\operatorname{tg} \neq 0 \text { for all } x \in \partial D \text { and } t \in[0,1],
$$

then there exist $n_{0} \geqq 1$ and $c>0$ such that

$$
\left\|T_{n}(x)-t Q_{n} g\right\| \geqq c \text { for } x \in \partial D_{n}, n \geqq n_{0}, t \in[0,1] .
$$

Before we discuss some special cases of Theorem $3.1 \mathrm{~K}$ we first observe that it admits a useful extension in which the $K$-coerciveness of $T$ is replaced by the more general

Condition $(3.1+)$. If $\left\{x_{n}\right\} \subset X$ is any sequence such that $T x_{n} \rightarrow g$ for some $g$ in $Y$, then $\left\{x_{n}\right\}$ is bounded. 
Note that Condition $(3.1+)$ is equivalent to the requirement that $T^{-1}(Q)=\{x \in X \mid T x \in Q\}$ be bounded whenever $Q$ is precompact in $Y$. Condition $(3.1+)$ is not only a weakening of (3.1-3) but also a proper weakening (see [127]) of the Condition (3.1\#) given by

CoNDition (3.1\#). $\|T x\| \rightarrow \infty$ as $\|x\| \rightarrow \infty$.

In view of this, mappings of monotone type and of ball-condensing type which satisfy Condition $(3.1+)$ have been recently studied by a number of authors (see [14], [41], [127], [134]).

It is not hard to show (see [127]) that if $r_{0}>0$ is given and $T: X \rightarrow Y$ satisfies Condition $(3.1+)$, then to each $f \in Y$ there correspond $r>r_{0}$ and $c>0$ such that

$$
\|T x-t f\| \geqq c \quad \text { for } x \in \partial B(0, r) \text { and } t \in[0,1] .
$$

In view of this and Lemma $3.1 \mathrm{~L}$, Theorem $3.1 \mathrm{~K}$ admits the following generalization (see [125]).

TheOREM 3.1M. Let $(X, Y)$ be Banach spaces, $K$ a map of $X$ into $Y^{*}$ for which (3.1-2) holds and $M_{n}$ a linear isomorphism of $X_{n}$ onto $Y_{n}$ for which (3.1-4) holds. Let $T: X \rightarrow Y$ be an A-proper mapping such that

(a) there exists $r_{0}>0$ with $(T x, K x) \geqq 0$ when $\|x\| \geqq r_{0}$.

Then, if $T$ satisfies Condition (3.1+), equation (3.1) is feebly approximation-solvable for each $f \in Y$. Equation (3.1) is strongly approximationsolvable if it is uniquely solvable for a given $f$.

We add in passing that Theorem $3.1 \mathrm{M}$ is applicable, in particular, to mappings $T: X \rightarrow Y$ satisfying any one of the conditions of Theorem $3.1 \mathrm{~J}$ provided that for $T$ satisfying either (J1) or (J2) we also assume that $T$ satisfies Condition $(3.1+)$ and the hypothesis (a) of Theorem 3.1M.

Let us observe that Theorem 3.1M allows us to study the approximationsolvability of the perturbed equations considered in [13], [112] of the form

$$
T x=A x+C x=f \quad(f \in Y)
$$

where $C$ is compact and $A$ is $A$-proper. In this case the conditions of Theorem 3.1 $\mathrm{M}$ are implied, for example, by the assumption:

(b) $A$ is $K$-coercive and there exists a constant $c_{0}$ such that $(C x, K x) \geqq$ $-c_{0}\|K x\|$ for $x \in X$.

REMARK 3.1-3. To apply the preceding results to equation (3.1) involving mappings $T$ from $X$ to $X^{*}$ or from $X$ to $X$ we have to choose $K$ and $M_{n}$ in some specified way. We illustrate this by the following discussion:

(i) In the first case we set $Y=X^{*}, \Gamma=\left\{X_{n}, P_{n} ; R\left(P_{n}^{*}\right), P_{n}^{*}\right\}$, choose $K=I$ and, if $\left\{\phi_{1}, \cdots, \phi_{n}\right\}$ is any basis in $X_{n}$, set $M_{n}(x)=\sum_{i=1}^{n}\left(\Phi_{i}, x\right) \Phi_{i}$ 
for $x \in X_{n}$, where $\left\{\Phi_{i}\right\} \subset R\left(P_{n}^{*}\right)$ is such that $\left(\Phi_{i}, \phi_{j}\right)=\delta_{i j}$, for $(1 \leqq i, j \leqq n)$. It follows that $K$ and $M_{n}$ thus chosen satisfy conditions (3.1-2) and (3.1-4).

(ii) In the second case we set $Y=X, \Gamma=\left\{X_{n}, P_{n} ; X_{n}, P_{n}\right\}$ and, assuming that $X$ is a $\Pi_{1}$-space with $X^{*}$ strictly convex, we may choose $M_{n}=I_{n}$ and $K=J$, where $J$ is any duality map of $X$ into $X^{*}$. Since by [22], $P_{n}^{*} J x=$ $J x$ for $x \in X_{n}$, we see that in this case (3.1-2) and (3.1-4) hold for the above choices of $M_{n}$ and $K$.

Of course, it is sometimes possible to choose a suitable linear mapping $K$ (see [105]) for which (3.1-2) and (3.1-4) hold. In that case the assumption in Lemma 3.1D and Theorem 3.1J that $K$ be weakly continuous at least at 0 is always true.

Before we state the next result which will illustrate the generality of Theorem $3.1 \mathrm{M}$ we first recall that following [6] we say that $F: X \rightarrow X$ is a generalized contraction on $X$ if to each $x$ in $X$ there is $\alpha(x) \in(0,1)$ such that $\|F x-F y\| \leqq \alpha(x)\|x-y\|$ for $y \in X$. One motivation for the extensive study of these maps $F$ is the fact proved by Kirk [65] that if its $F$-derivative $F_{x}^{\prime}$ is continuous in $x$, then $F$ is a generalized contraction if and only if $\left\|F_{x}^{\prime}\right\|<1$. It was shown by Wong [158] (see also [39]) that if $X$ is a reflexive $\Pi_{1}$-space and $F: X \rightarrow X$ is a generalized contraction, then $F$ is $P_{1^{-}}$ compact and, in particular, $T=I-F$ is $A$-proper. Moreover, $T$ is injective and coercive.

In view of the above discussion, Theorem 3.1J and the discussion in $\S 1.1$, Theorem $3.1 \mathrm{M}$ implies the validity of the following corollary which unites and extends a number of results obtained in [9], [19], [42], [115], [119] for various classes of mappings by using different arguments. We add that the perturbed problems considered in the next corollary cannot be handled by the methods employed by the above mentioned authors. As before, it is always assumed that a couple of Banach spaces and a projectionally complete scheme are given.

CoRollary 3.1N. (A1) Let $X$ be reflexive and $T: X \rightarrow X^{*}$ a continuous mapping of modified type (S) such that $T$ satisfies condition $(3.1+)$ and $(T x, x) \geqq 0$ for all $x \in X-B(0, r)$ and some $r>0$. Then equation (3.1) is feebly approximation-solvable for each $f \in X^{*}$, and strongly approximationsolvable if (3.1) is uniquely solvable.

(A2) Suppose $C: X \rightarrow X^{*}$ is compact and $(C x, x) \geqq-c_{0}\|x\|$ for all $x \in X$ and some constant $c_{0}$. If $X$ is reflexive with property $(\mathrm{H})$ and $A: X \rightarrow X^{*}$ is continuous and satisfies condition (J2) of Theorem 3.1 $\mathrm{J}$ with $K=I$ (and $T=A$ ), then equation (3.1-8) is feebly approximation-solvable for each $f \in X^{*}$, and strongly approximation-solvable if (3.1-8) is uniquely solvable.

(A3) Let $X$ be a Banach $\Pi_{1}$-space with $X^{*}$ strictly convex and $F: X \rightarrow X$ ball-condensing such that $(F x, J x) \leqq(x, J x)$ for all $x \in X-B\left(0, r_{0}\right)$ and 
some $r_{0}>0$, where $J$ is any single valued duality map of $X$ into $X^{*}$. If $T=I-F$ satisfies condition $(3.1+)$, then equation (3.1) is feebly approximationsolvable for each $f$ in $X$, and strongly approximation-solvable if (3.1) is uniquely solvable.

(A4) Let $X$ be a reflexive $\Pi_{1}$-space with $X^{*}$ strictly convex and $C: X \rightarrow X$ a compact map with $(C x, J x) \geqq-c_{0}\|x\|$ for all $x \in X$ and some constant $c_{0}$. If $F: X \rightarrow X$ is a generalized contraction, then equation (3.1-8) (with $A=I-F$ ) is feebly approximation-solvable for each $f \in X$, and strongly approximationsolvable if (3.1-8) is uniquely solvable.

(A5) Let $\left(X, X^{*}\right)$ be uniformly convex, $X$ a $\Pi_{1}$-space, $J: X \rightarrow X^{*} a$ normalized duality map, and $C: X \rightarrow X$ compact with $(C x, J x) \geqq-c_{0}\|x\|$ for all $x \in X$ and some constant $c_{0}$. If $A: X \rightarrow X$ satisfies the conditions of Corollary $3.1 \mathrm{H}$ for $K=J$, then equation (3.1-8) is feebly approximation-solvable for each $f \in X$, and strongly approximation-solvable if (3.1-8) is uniquely solvable.

Remark 3.1-4. Assertion (A1) is also valid if $T$ is assumed to be bounded, demicontinuous and of type (S). Similarly, (A2) is valid if $A$ is assumed to be bounded and demicontinuous.

An application to elliptic $B V P$. Let $W_{p}^{m}$ and $\mathscr{W}_{p}^{m}$ be the Sobolev spaces introduced in \$2.4. For a given $f \in L_{\alpha}$, the generalized Dirichlet BVP for

$$
\begin{array}{r}
\sum_{|\alpha| \leqq m}(-1)^{|\alpha|} D^{\alpha}\left(\left|D^{\alpha} u\right|^{p-2} D u\right)+\sum_{|\beta| \leqq m-1}(-1)^{|\beta|} D^{\beta} B_{\beta}\left(x, u, \cdots, D^{m} u\right) \\
D^{\alpha} u=0 \text { on } \partial Q \text { for }|\alpha| \leqq m-1
\end{array}
$$

consists in finding a weak solution of (3.1-9), i.e., $u \in \stackrel{\circ}{W}_{p}^{m}$ such that

$$
a(u, v)+b(u, v)=\langle f, v\rangle \text { for all } v \text { in } \stackrel{\circ}{W}_{p}^{m},
$$

where the Dirichlet forms $a(u, v)$ and $b(u, v)$ are given by

$$
\begin{aligned}
& a(u, v)=\sum_{|\alpha| \leqq m}\left\langle\left|D^{\alpha} u\right|^{p-2} D^{\alpha} u, D^{\alpha} v\right\rangle \quad\left(u, v \in \stackrel{\circ}{W}_{p}^{m}\right) \\
& b(u, v)=\sum_{|\beta| \leqq m-1}\left\langle B_{\beta}\left(x, u, \cdots, D^{m} u\right), D^{\beta} v\right\rangle \quad\left(u, v \in \stackrel{\circ}{W}_{p}^{m}\right) .
\end{aligned}
$$

It is easy to show that $a(u, v)$ is well defined and in order for $b(u, v)$ to be well defined we make the following

Assumption I. The functions $B_{\beta}(x, \xi)$, where $\xi=\left\{\xi_{\alpha}:|\alpha| \leqq m\right\}$ is a vector in some Euclidean space $R^{M}$, are continuous in $\xi$ for fixed $x$ and measurable in $x$ for fixed $\xi$. Moreover, there exists a real $p \in[2, \infty)$ and a constant $c>0$ such that $\left|B_{\beta}(x, \xi)\right| \leqq c\left(1+|\xi|^{p-1}\right)$ for all $x \in Q$ and all $\beta$. 
It follows from Assumption I, using Hölder's inequality, that $b(u, v)$ is well defined on $\mathscr{W}_{p}^{m}$ (see [14], [81]). Setting $X=\mathscr{W}_{p}^{m}$, we see that $a(u, v)$ and $b(u, v)$ are continuous linear functionals of $v$ in $X$ and so they determine unique bounded continuous mappings $A, B: X \rightarrow X^{*}\left(=W_{a}^{-m}\right)$ such that

$(3.1-13) \quad a(u, v)=(A u, v)$ and $b(u, v)=(B u, v)$ for all $v \in X$.

Denoting the norms in $X=\stackrel{\circ}{W_{p}^{m}}$ and in $X^{*}$ by $\|\cdot\|$, we find as in [9] that for $u$ and $v$ in $X$

$$
|(A u, v)| \leqq\|u\|^{p-1}\|v\|, \quad(A u, u)=\|u\|^{p}, \quad(A u, u)=\|A u\|\|u\| .
$$

It follows from (3.1-14) that $A$ is coercive and together with (3.1-13) that

$$
(A u-A v, u-v) \geqq(\phi(\|u\|)-\phi(\|v\|))(\|u\|-\|v\|) \text { for } u, v \in X,
$$

where $\phi(r)=r^{p-1}, r \geqq 0$. Since, by the Sobolev imbedding theorem, the injection of $\mathscr{W}_{p}^{m}$ into $W_{p}^{m-1}$ is compact, it is not hard to show (see [14], [123]) that $B$ defined by (3.1-13) is compact.

The above discussion implies that the generalized Dirichlet BVP for (3.1-9) is equivalent to the solvability of the equation

$$
A u+B u=w_{f} \quad\left(u \in X, w_{f} \in X^{*}\right),
$$

where $w_{f}$ is the unique element in $X^{*}$ such that $\left(w_{f}, v\right)=\langle f, v\rangle$ for all $v \in X$. To apply (A2) of Corollary $3.1 \mathrm{~N}$ to equation (3.1-15) we need the following

Assumption II. There exists a constant $c_{0}$ such that $b(u, u) \geqq-c_{0}\|u\|_{m, p}$ for all $u$ in $\stackrel{\circ}{W}_{p}^{m}$.

If $\left\{\phi_{i}\right\} \subset \mathscr{W}_{p}^{m}$ is a Schauder basis (see [45]) and $\Gamma_{s}$ is the corresponding scheme for $\left(X, X^{*}\right)$, then (A2) of Corollary $3.1 \mathrm{~N}$ implies the approximation-solvability of equation (3.1-15) (i.e., of (3.1-9)) in the form:

THEOREM 3.10. Suppose that Assumptions I and II are satisfied. Then the generalized Dirichlet BVP for (3.1-9) is feebly approximationsolvable with respect to $\Gamma_{s}$ for each $f \in L_{q}$ and, in particular, the problem (3.1-9) has a weak solution for each $f \in L_{a}$. It is strongly approximationsolvable for $f \in L_{a}$ if it is uniquely solvable.

3.2. Equations involving odd A-proper mappings. In this section we discuss the approximation-solvability of equation (3.1) involving odd mappings but without the assumption that $T$ is $a$-stable or $K$-coercive.

Recall that $T: X \rightarrow Y$ is odd on $X$ if $T(-x)=-T(x)$ for $x \in X . T$ is $\alpha$-homogeneous if $T(t x)=t^{\alpha} T(x)$ for all $x \in X$, all $t \geqq 0$ and some $\alpha>0$. 
If $X$ is a real Banach space, $C$ a compact linear map of $X$ into $X$ and $\lambda \neq 0$ not an eigenvalue of $C$, then the classical result (see [64]) asserts that $(\lambda I-C)(X)=X$. In [130] Pokhodjayev extended this result to certain nonlinear mappings by showing

THEOREM 3.2A. If $X$ is a real reflexive Banach space with a Schauder basis and $A, C: X \rightarrow X^{*}$ are odd, $\alpha$-homogeneous and such that $C$ is compact and $A$ is bounded, continuous and strongly closed, then $(\lambda A-C)(X)=X^{*}$ whenever $\lambda \neq 0$ and $\lambda A x-C x \neq 0$ for $x \neq 0$.

We add that for a reflexive space $X$ with a Schauder basis the strongly closed maps $T: X \rightarrow X^{*}$, as defined in [130], are $A$-proper with respect to $\Gamma_{S}$. Theorem 3.2A was then used in [130] to obtain existence results for generalized Dirichlet BVP for quasi-nonlinear elliptic equations involving odd and $\alpha$-homogeneous operators. In [115] the author extended Theorem 3.2A to obtain a direct analogue of the classical linear result. We state Theorem 1.4 from [115] for the case when $(X, Y)$ are real Banach spaces with a given projectionally complete scheme $\Gamma$.

Theorem 3.2B. Suppose $A, C: X \rightarrow Y$ are odd, and $\alpha$-homogeneous, $C$ is compact and $A$ is $A$-proper. If $\lambda \neq 0$ and $\lambda A x-C x \neq 0$ for $x \neq 0$, then the equation

$$
\lambda A x-C x=f \quad(f \in Y)
$$

is feebly approximation-solvable for each $f \in Y$. Equation (3.2-1) is strongly approximation-solvable if it is uniquely solvable.

The proof of Theorem 3.2B is based on Lemma 3.1L and the following proposition which is of interest in its own right.

Proposition 3.2C. Let $D$ be a bounded open subset of $X$ which is symmetric about $0 \in D$ and let $T: \bar{D} \rightarrow Y$ be odd and A-proper.

If $f$ is a given element in $Y$ such that

$$
T x-t f \neq 0 \text { for } x \in \partial D \text { and } t \in[0,1],
$$

then the equation

$$
T x=f \quad(x \in D, f \in Y)
$$

is feebly approximation-solvable in $D$, and strongly approximation-solvable if (3.2-3) is uniquely solvable.

We add in passing that Proposition 3.2C includes the fixed point theorem for odd $P_{1}$-compact mappings and, in particular, for ball-condensing and compact mappings. 
In view of Proposition 3.2C, Theorem 3.2B admits the following generalization established by the author in [120].

Theorem 3.2D. Suppose $T: X \rightarrow Y$ is $A$-proper and odd on $X-B(0, r)$ for some $r \geqq 0$. Suppose further that

(D1) To each $f \in Y$ there corresponds $r_{f} \geqq r$ such that $T x-t f \neq 0$ for $x \in \partial B\left(0, r_{f}\right)$ and $t \in[0,1]$.

Then equation (3.1) is feebly approximation-solvable for each $f \in Y$. Equation (3.1) is strongly approximation-solvable if it is uniquely solvable.

For Theorem 3.2D to be useful in applications, it is important to find practically verifiable conditions which would imply the validity of the hypothesis (D1). In view of the inequality (3.1-7), it seems that condition $(3.1+)$ is the most suitable (and, in fact, the most general) for our purposes. Consequently, in virtue of (3.1-7), Theorem 3.2D implies the validity of the thus far most general and practically useful corollary.

Corollary 3.2E. Suppose $T: X \rightarrow Y$ is $A$-proper and odd on $X-B(0, r)$ for some $r \geqq 0$. If $T$ satisfies condition $(3.1+)$, then equation (3.1) is feebly approximation-solvable for each $f$ in $Y$. Equation (3.1) is strongly approximation-solvable if it is uniquely solvable.

Remarks 3.2-1. (i) Since the condition (\#) (i.e., $\|T x\| \rightarrow \infty$ as $\|x\| \rightarrow \infty$ ) as well as the condition (\#\#): " $0 \notin T(\partial B(0, r))$ and $T(t x)=t^{\alpha} T(x)$ for all $\|x\| \geqq r, t>1$ and some $\alpha>0$ " are both properly stronger than Condition $(3.1+)$, Theorem 1 in [120] follows from Corollary 3.2E.

(ii) Note that Corollary 3.2E applies to mappings $T: X \rightarrow Y$ satisfying either condition (J1) or (J3) of Theorem 3.1J if $T$ is also odd on $X-B(0, r)$ and satisfies Condition $(3.1+)$.

(iii) If $F: X \rightarrow X$ is $P_{1}$-compact and, in particular, ball-condensing and such that $F$ is odd on $X-B(0, r)$ and $T=I-F$ satisfies Condition $(3.1+)$, then Corollary 3.2E applies. In view of this, a number of existence theorems obtained in [127] and [157] can be imbedded into the constructions theory of $A$-proper mappings under conditions similar to those used in [127], [157].

3.3. Fredholm alternative for asymptotically linear A-proper maps. Following [67] we say that a nonlinear map $T: X \rightarrow Y$ is asymptotically linear if there exists a linear map $T_{\infty} \in L(X, Y)$, called the asymptotic derivative of $T$, such that for all $x \in X$

(3.3-1) $\quad T(x)=T_{\infty}(x)+B(x)$ with $\|B x\| /\|x\| \rightarrow 0$ as $\|x\| \rightarrow \infty$.

In [67] Krasnoselsky initiated the study of equations

$$
x-C x=f \quad(x \in X, f \in X),
$$


where $C$ is compact and asymptotically linear, which was then continued by him and other authors (see [67], [4] for references).

In [58] Kachurovsky extended (without proof) the classical Fredholm alternative to equation (3.3-2) with $C$ compact and asymptotically linear. In [126] the writer extended the result of [58] to the case when $C$ is asymptotically linear and $k$-ball-contractive with $k<1$ and then used it to obtain existence of classical solutions for nonlinear elliptic boundary value problems. An alternative, which also extends [58] in case $X$ is a Hilbert space, has been proved by Hess [53] for the case when $X$ is reflexive and $T: X \rightarrow X^{*}$ is a bounded, demicontinuous, and asymptotically linear map of type (S) with $T_{\infty}$ also of type (S). He applied his result to the generalized Dirichlet BVP for an elliptic second order equation.

In [123] the writer extended the Fredholm alternative for linear $A$ proper mappings (i.e., Theorem 2.3A) to equations

$$
T(x)=f \quad(x \in X, f \in Y)
$$

involving an $A$-proper and asymptotically linear mapping $T: X \rightarrow Y$. The application of our alternative to nonlinear elliptic boundary value problems were also given in [123].

The basic result in this section is the following alternative in [123] extending Theorem 2.3A to nonlinear maps which, as will be seen, provides also a constructive generalization and unification of the alternatives in [53], [58], [91], [92], [126].

THEOREM 3.3A. Let $T: X \rightarrow Y$ be an asymptotically linear A-proper mapping with an A-proper asymptotic derivative $T_{\infty} \in L(X, Y)$. Then either equation (3.3-3) is feebly approximation-solvable for each $f$ in $Y$ (and strongly approximation-solvable if $T$ is injective), or $N\left(T_{\infty}\right) \neq\{0\}$. In the latter case, assuming additionally that $\operatorname{dim} N\left(T_{\infty}\right)=\operatorname{codim} R\left(T_{\infty}\right)$ and that

$$
B x \in N\left(T_{\infty}^{*}\right)^{\perp} \text { for } x \in X \text {, where } B=T-T_{\infty},
$$

or equivalently that

$$
R(T) \subset R\left(T_{\infty}\right)
$$

equation (3.3-3) is solvable if and only if $f \in N\left(T_{\infty}^{*}\right)^{\perp}\left(=R\left(T_{\infty}\right)\right)$.

The proof of Theorem 3.3A is based upon Theorem 2.3A, the finite dimensional Brouwer degree theory, and the following proposition which includes Proposition 1 in [53] for the type of spaces considered here.

Proposition 3.3B. Let $T: X \rightarrow Y$ be A-proper with the A-proper asymptotic derivative $T_{\infty}$. If $N\left(T_{\infty}\right)=\{0\}$, then equation (3.3-3) is feebly approximation-solvable for each $f$ in $Y$. Moreover, equation (3.3-3) is strongly 
approximation-solvable if it is uniquely solvable (i.e., the Galerkin type method is convergent in this case).

REMARK 3.3-1. (i) If $Y=X, X$ is a $\Pi_{1}$-space, $C: X \rightarrow X$ asymptotically linear and $k$-ball-contractive with $k<1$, then $C_{\infty}$ is also $k$-ball-contractive (see [126]) and so $T=I-C$ and $T_{\infty}=I-C_{\infty}$ are $A$-proper by the results in [93], [156]. Moreover, it was shown in [94] that $i(T)=0$. The above holds, in particular, when $C$ is compact. Hence Theorem $3.3 \mathrm{~A}$ is applicable to equation (3.3-2). This implies in particular, the validity of the alternatives in [58], [126].

(ii) If $X$ is reflexive with $\left(X, X^{*}\right)$ having a projectionally complete scheme $\Gamma_{1}$ and $T: X \rightarrow X^{*}$ is bounded, demicontinuous, and asymptotically linear of type (S) with $T_{\infty}$ also of type (S), then $T$ and $T_{\infty}$ are $A$-proper. Now, since $X$ is reflexive, $T_{\infty}: X \rightarrow X^{*}$ is of type (S) if and only if $T_{\infty}^{*}: X \rightarrow X^{*}$ is of type (S). Hence, $T_{\infty}^{*}$ is also $A$-proper and therefore, by Theorem 2.3B, $i\left(T_{\infty}\right)=0$. Consequently, Theorem $3.3 \mathrm{~A}$ is applicable to equation (3.3-3) for $Y=X^{*}$ and $T$ satisfying the above conditions. This implies, in particular, the alternative in [53].

(iii) We add that in [91], [92] Nečas studied the surjectivity of $T: X \rightarrow X^{*}$ of the form $T=A+B$, where $B$ is asymptotically zero and $A$ is of type (S) and $\alpha$-homogeneous with either $A$ or $T$ odd. It was shown in [123] that the results of Nečas can also be deduced from the corresponding results for $A$-proper mappings (see [123, Theorem 2]).

(iv) The term "Fredholm alternative" is obviously not unambiguous in its uses for nonlinear operators. In particular, the present considerations have no point of contact with recent results of Pokhadjayev, Browder and others concerning the concept of "normal solvability" or "Fredholm alternative" for a class of differentiable mappings.

Application of Theorem 3.3A to asymptotically linear elliptic BVP. Consider the approximation-solvability of the generalized Dirichlet BVP in $\mathscr{W}_{2}^{m} \equiv \mathscr{W}_{2}^{m}(Q)$ for the nonlinear elliptic equation of order $2 m$

$$
\begin{aligned}
\sum_{|\alpha|,|\beta| \leq m}(-1)^{|\alpha|} D^{\alpha}\left(a_{\alpha \beta}(x) D^{\beta} u\right) & \\
& +\sum_{|\beta| \leq m-1}(-1)^{|\beta|} D^{\beta} B_{\beta}\left(x, u, \cdots, D^{m} u\right)=f(x),
\end{aligned}
$$

where the linear part is strongly elliptic with $a_{\alpha \beta}$ satisfying the conditions of $\$ 2.4$ and the functions $B_{\beta}(x, \xi)$ of the nonlinear part satisfy Assumption I of $\$ 3.1$ for $p=2$. For a given $f$ in $L_{2}$, the generalized Dirichlet BVP for (3.3-6) calls for $u \in \mathscr{W}_{2}^{m}$ such that

$$
l(u, v)+b(u, v)=\langle f, v\rangle \text { for all } v \in \stackrel{\circ}{W}_{2}^{m},
$$


where $l(u, v)$ is given by $(2.4-5)$ and $b(u, v)$ by (3.1-12). The conditions on $a_{\alpha \beta}$ and $B_{\beta}(x, \xi)$ imply that

$$
l(u, v)=(L u, v)_{m} \quad \text { and } \quad b(u, v)=(B u, v)_{m} \quad\left(u, v \in \stackrel{\circ}{W}_{2}^{m}\right),
$$

where $L, B: \stackrel{\circ}{W}_{2}^{m} \rightarrow \stackrel{\circ}{W}_{2}^{m}$ are bounded and continuous with $L$ linear and $B$ nonlinear and compact (see \$3.1). In view of this and the equality $\langle f, v\rangle=\left(w_{f}, v\right)$ for a unique $w_{f} \in \mathscr{\circ}_{2}^{m}$ and all $v$ in $\stackrel{\circ}{W}_{2}^{m},(3.3-7)$ is equivalent to

$$
L u+B u=w_{f} \quad\left(u, w_{f} \in \stackrel{\circ}{W}_{2}^{m}\right) .
$$

For Theorem 3.3A to be applicable to (3.3.6) we also impose (see B2 in [123]):

Assumption III. There exist $b_{\beta \gamma}(x) \in L^{\infty}(\bar{Q})$ for $|\beta| \leqq m-1$ and $|\gamma| \leqq m$ and a continuous function $d: R^{+} \rightarrow R^{+}$with $d(t) / t \rightarrow 0$ as $t \rightarrow \infty$ such that

$$
\left|B_{\beta}\left(x, u, \cdots, D^{m} u\right)-\sum_{|\gamma| \leq m} b_{\beta \gamma}(x) D^{\gamma} u\right| \leqq d\left(\|u\|_{m, 2}\right) \text { for all } u \in \stackrel{\circ}{W}_{2}^{m} \text {. }
$$

Along with (3.3-6) we consider the generalized Dirichlet BVP for

$$
\sum_{|\alpha| .|\beta| \leq m}(-1)^{|\alpha|} D^{\alpha}\left(a_{\alpha \beta} D^{\beta} u\right)+\sum_{|\beta| \leq m-1,|\gamma| \leq m}(-1)^{|\beta|} D^{\beta}\left(b_{\beta \gamma} D^{\gamma} u\right)=0 .
$$

Now, Assumption III implies the existence of $M \in L\left(\stackrel{\circ}{W}_{2}^{m}, \stackrel{\circ}{W}_{2}^{m}\right)$ given by

$$
(M u, v)_{m}=m(u, v)=\sum_{|\beta| \leq m-1,|\gamma| \leq m}\left\langle b_{\beta \gamma}(x) D_{u}^{\gamma}, D_{v}^{\beta}\right\rangle \quad\left(u, v \in \mathscr{W}_{2}^{m}\right)
$$

such that $M$ is compact, since $|m(u, u)| \leqq d_{0}\|u\|_{m, 2}\|u\|_{m-1,2}$ for $u \in \stackrel{\circ}{W}_{2}^{m}$, and

$$
\frac{\left\|T(u)-T_{\infty}(u)\right\|_{m, 2}}{\|u\|_{m, 2}}=\frac{\|B u-M u\|_{m, 2}}{\|u\|_{m, 2}} \rightarrow 0 \text { as }\|u\|_{m, 2} \rightarrow \infty,
$$

where $T=L+B$ and $T_{\infty}=L+M$. In view of the Gårding inequality (2.4-10), $L$ is $A$-proper with respect to any given projectionally complete scheme $\Gamma_{0}=\left(X_{n}, P_{n} ; X_{n}, P_{n}\right)$ for $\left(\stackrel{\circ}{W}_{2}^{m}, \stackrel{\circ}{W}_{2}^{m}\right)$ and so are the mappings $T$ and $T_{\infty}$. Since $T_{\infty}^{*}=L^{*}+M^{*}$ is also $A$-proper, Theorem 2.3B implies that $i\left(T_{\infty}\right)=0$. Consequently, all the conditions of Theorem 3.3A are satisfied and therefore we deduce from the latter the following new results for the generalized Dirichlet BVP for (3.3-6) under the above conditions on the functions $a_{\alpha \beta}(x), b_{\beta \gamma}(x)$ and $B_{\beta}(x, \xi)$ (see [123]), which provide analogues to Theorems $2.4 \mathrm{~A}$ and $2.4 \mathrm{~B}$. 
THEOREM 3.3C. If the generalized Dirichlet BVP for linear equation (3.3-10) has only null solutions in $\mathscr{W}_{2}^{m}$ (i.e., $N\left(T_{\infty}\right)=\{0\}$ ), then the generalized Dirichlet BVP for the nonlinear equation (3.3-6) is feebly approximation-solvable for each $f$ in $L_{2}$ (and, in particular, it has a weak solution for each $f$ in $\left.L_{2}\right)$. It is strongly approximation-solvable if for a given $f$ in $L_{2}$ it has a unique weak solution (i.e., the Galerkin type method for (3.3-6) converges in $\stackrel{\circ}{W}_{2}^{m}$ ).

THEOREM 3.3D. If $N\left(T_{\infty}\right) \neq\{0\}$ and if $v_{1}, \cdots, v_{k}$ is a basis for $N\left(T_{\infty}^{*}\right) \subset$ $\stackrel{\circ}{W}_{2}^{m}$ with $k=\operatorname{dim} N\left(T_{\infty}\right)$, then under the additional condition

$$
\sum_{|\beta| \leq m-1}\left\langle B_{\beta}\left(x, u, \cdots, D^{m} u\right)-\sum_{|\gamma| \leq m} b_{\beta \gamma} D^{\gamma} u, D^{\beta} v_{k}\right\rangle=0 \quad(1 \leqq j \leqq k),
$$

the generalized Dirichlet BVP for (3.3-6) is solvable in $\stackrel{\circ}{W}_{2}^{m}$ for a given $f$ in $L_{2}$ if and only if $\left\langle f, v_{j}\right\rangle=0$ for $1<j \leqq k$.

REMARK 3.3-2. Theorems 3.3C and 3.3D include the existence Theorems 6 and 7 of Kachurovsky [59] obtained by him by other methods for the case when $N_{\beta}$ depends on $x$ and $D^{\alpha} u$ with $|\alpha| \leqq m-1$ but not on $D^{\alpha} u$ with $|\alpha|=m$ and where $l(u, u)$ is assumed to be positive definite, i.e., $l(u, u) \geqq c_{1}\|u\|_{m, 2}^{2}$ for $u$ in $\stackrel{\circ}{W}_{2}^{m}$ and some $c_{1}>0$. Our theorems also include the existence Theorem 3 of Hess [53] obtained by him for the case when $m=1$. For other applications of Theorem 3.3A see [123].

\section{Generalized degree theory for $A$-proper mappings and applications}

If $D \subset X$ is open and bounded, $T: \bar{D} \rightarrow Y$ satisfies suitable conditions and $f \notin T(\partial D)$, then the topological degree of $T$ on $D$ over $f, \operatorname{deg}(T, D, f)$, is in principle an algebraic count of the number of solutions $x \in D$ of $T x=f$. For this count to be useful, it must have several crucial properties: additivity on the domain $D$, invariance under suitable homotopies of $T$, existence of a solution $x$ in $D$ of $T x=f$ if $\operatorname{deg}(T, D, f) \neq 0$, etc.

In [80] Leray and Schauder extended the Brouwer degree to $T: \bar{D} \subset$ $X \rightarrow X$ of the form $T=I+C$ with $C$ compact. To carry through this extension, they used the uniform approximations of $C$ by finite dimensional maps. In the recent development of nonlinear functional analysis and, in particular, in connection with the theory of nonlinear operators like operators of monotone and accretive type, operators of ultimately compact and condensing type and operators of $A$-proper type, it has become an important objective to widen the scope of the degree theory beyond that of the Leray-Schauder degree. Degree theories of more general types were constructed for general classes of maps in Borisovich and Sapronov [7], Browder [16], [20], Browder and Nussbaum [23], Browder and Petryshyn [24], [25], Fitzpatrick [38], Nussbaum [93], Petryshyn and Fitzpatrick [128], Sadovsky [136], Skrypnyk [142], Wong [158], and others. 
In [24], [25] Browder and Petryshyn introduced and studied the notion of the topological degree for an $A$-proper mapping $T: \bar{D} \subset X \rightarrow Y$ in the context of constructive functional analysis. We generalized the LeraySchauder method by replacing uniform approximation by finite-dimensional mappings by much more general approximation methods which, among others, include projectional and injective methods of Galerkin type. This degree theory was then further studied and applied by many authors, including Browder [16], [19], Deimling [30], Fitzpatrick [39], [42], O'Neil and Thomas [97], Nussbaum [43], Petry [100], Petryshyn [117], [119], Potter [133], Stuart [144], Webb [156], Wong [158], and others.

In $\$ 4.1$ we define and outline the basic properties of the generalized degree while in $\$ 4.2$ the degree theory is applied to various fixed point and mapping theorems. In $\$ 4.3$ we outline some results for Fréchet differentiable $P$-compact and $A$-proper mappings as well as some results for $A$-proper mappings of analytic type. New approximation-solvability results are outlined.

4.1. Definition and properties of the generalized degree. As before we assume that $(X, Y)$ are real Banach spaces with an oriented projectionally complete scheme $\Gamma=\left\{X_{n}, P_{n} ; Y_{n}, Q_{n}\right\}$ for $(X, Y)$ with respect to which the degree of $T$ is given by the following definition.

Definition 4.1A. Let $D$ be a bounded open subset of $X, T: \bar{D} \rightarrow Y$ $A$-proper and $f \in Y-T(\partial D)$. Let $Z^{\prime}=Z \cup\{+\infty,-\infty\}$, where $Z$ denotes the set of integers. We define $\operatorname{Deg}(T, D, f)$, the degree of $T$ on $D$ over $f$ with respect to $\Gamma$, as a subset of $Z^{\prime}$ given by:

(A1) The integer $m \in \operatorname{Deg}(T, D, f)$ if there exists an infinite sequence $\left\{n_{j}\right\}$ such that $\operatorname{deg}\left(T_{n}, D_{n_{i}}, Q_{n} f\right)$ is well defined and equals $m$ for each $j$.

(A2) $+\infty(-\infty) \in \operatorname{Deg}(T, D, f)$ if there exists an infinite sequence $\left\{n_{j}\right\}$ such that $\operatorname{deg}\left(T_{n_{j}}, D_{n_{j}}, Q_{n_{j}} f\right)$ is well defined for each $j$ and $\lim _{j} \operatorname{deg}\left(T_{n_{j}}, D_{n_{j}}, Q_{n_{f}} f\right)=+\infty(-\infty)$.

(The degree $\operatorname{deg}\left(T_{n}, G_{n}, Q_{n} f\right)$ used in Definition 4.1A is the classical Brouwer degree for mappings of oriented finite dimensional Euclidean spaces of the same dimension.)

Utilizing the properties of the Brouwer degree and of $A$-proper mappings, it was shown in [24], [25] that although, in general, $\operatorname{Deg}(T, D, f)$ is multivalued it has most of the useful properties of the Brouwer degree as the following theorem indicates.

THEOREM 4.1B. Let $D$ be a bounded open subset of $X, T: \bar{D} \rightarrow Y$ A-proper and $f$ a point in $Y-T(\partial D)$. Then:

(B1) There exists an integer $n_{0} \geqq 1$ such that $Q_{n} f \notin T_{n}\left(\partial D_{n}\right)$ for $n \geqq n_{0}$. Hence for such $n, \operatorname{deg}\left(T_{n}, D_{n}, Q_{n} f\right)$ is well defined and, in particular, $\operatorname{Deg}(T, D, f)$ is a nonempty subset of $Z^{\prime}$. 
(B2) If $\operatorname{Deg}(T, D, f) \neq\{0\}$, then there is $x \in D$ such that $T x=f$.

(B3) Let $H: \bar{D} \times[0,1] \rightarrow Y$ be continuous in $t$, uniformly with respect to $x \in \partial D$ and such that $H(\cdot, t): \bar{D} \rightarrow Y$ is A-proper for each $t \in[0,1]$. Then, if $f \in Y$ is such that $H(x, t) \neq f$ for $x \in \partial D$ and $t \in[0,1], \operatorname{Deg}(H(\cdot, t), D, f)$ is independent of $t$ in $[0,1]$.

(B4) Let $D=D_{1} \cup D_{2}, D^{\prime}=\left(D_{1} \cap D_{2}\right) \cup \partial D_{1} \cup \partial D_{2}$, and $f \notin T\left(D^{\prime}\right)$. Then $\operatorname{Deg}(T, D, f) \subseteq \operatorname{Deg}\left(T, D_{1}, f\right)+\operatorname{Deg}\left(T, D_{2}, f\right)$, with equality holding if either $\operatorname{Deg}\left(T, D_{1}, f\right)$ or $\operatorname{Deg}\left(T, D_{2}, f\right)$ is single-valued. (If $A_{1}, A_{2} \subset Z^{\prime}$, then $A_{1}+A_{2}=\left\{a \mid a=a_{1}+a_{2}, a_{1} \in A_{1}, a_{2} \in A_{2}\right\}$ and we use the convention that $+\infty+(-\infty)=Z^{\prime}$.)

(B5) Suppose $0 \in D$ and $D$ is also symmetric about 0 . If $T: \bar{D} \rightarrow Y$ is $A$ proper with $T_{n}$ odd on $\partial D_{n}$ for each $n$ and $0 \notin T(\partial D)$, then $\operatorname{Deg}(T, D, 0)$ is odd (i.e., $2 m \notin \operatorname{Deg}(T, D, 0)$ for any integer $m)$ and, in particular, $\operatorname{Deg}(T, D, 0) \neq$ $\{0\}$ so that $T x=0$ has a solution in $D$.

REMARK 4.1-1. The following observation is, perhaps, in order. In defining $\operatorname{Deg}(T, D, f)$ it was assumed in [24], [25] that $D$ was open with $\bar{D}$ a closure in $X$ and $T: \bar{D} \rightarrow Y$ was continuous. However, in the proof of Theorem 4.1B these assumptions were not really used. The only conditions that were used are that $D_{n}=X_{n} \cap D$ is an open set in $X_{n}$ with closure $\bar{D}_{n}$ and boundary $\partial D_{n}$ in $X_{n}$, that $T_{n}: \bar{D}_{n} \subset X_{n} \rightarrow Y_{n}$ is continuous for each $n$, and that $\bar{D}_{n} \subset \bar{D}$ and $\partial D_{n} \subset \partial D$ for all $n$ where $\bar{D}$ is not necessarily the closure of $D$ in $X$ or $\partial D$ the boundary of $D$ in $X$. It was noted in [39] that the homotopy property holds under the following weaker condition which will prove to be useful in applications:

$\left(\mathrm{B3}^{\prime}\right)$ Let $H: \bar{D} \times[0,1] \rightarrow Y$ be such that for each $n$ the map $H_{n}: \bar{D}_{n} \times$ $[0,1] \rightarrow Y_{n}$ is continuous in $t$, uniformly for $x \in \partial D_{n} ; H(t, \cdot)$ is $A$-proper for each $t \in[0,1]$ and if $\left\{x_{n_{j}} \in \partial D_{n_{j}}\right\}$ and $\left\{t_{n_{j}}\right\} \subset[0,1]$ are such that $Q_{n_{j}} H\left(x_{n_{j}}, t_{n_{j}}\right)$ $\rightarrow g$ in $Y$, then there are subsequences $\left\{x_{n_{j(k)}}\right\}$ and $\left\{t_{n_{j(k)}}\right\}$ such that $x_{n_{j(k)}} \rightarrow$ $x, t_{n_{j(k)}} \rightarrow t$ and $H(x, t)=g$. Then, if $f \in Y$ and $H(x, t) \neq f$ for $x \in \partial D$ and $t \in[0,1], \operatorname{Deg}(T, D, f)$ is independent of $t \in[0,1]$.

Theorem 4.1B has as corollaries a number of interesting fixed point and mapping theorems for $A$-proper mappings, some of which will be mentioned here and others in $\$ \$ 4.2$ and 4.3.

An immediate and useful consequence of (B3) and the properties of linear $A$-proper mappings is the following corollary (see also [158]).

Corollary 4.1C. If $T \in L(X, Y)$ is A-proper and injective, then to each $f \in R(T)$ there corresponds $B(0, r)$ such that $\operatorname{Deg}(T, B(0, r), f) \subset\{ \pm 1\}$.

Another useful result for $T \in L(X, Y)$ obtained by Fitzpatrick [42] will play an important role in the computation of the degree of an $A$ proper mapping $T$ in terms of its Fréchet derivative $T^{\prime}$. 
Proposition 4.1D. If $T \in L(X, Y)$ is A-proper and injective, then $\operatorname{Deg}(T, B(0, r), 0)=\operatorname{Deg}\left(T, B\left(x_{0}, r\right), T\left(x_{0}\right)\right)$ for any $x_{0} \in X$ and $r>0$.

The following result from [24] may prove to be useful in applications.

Proposition 4.1E. If $T: \bar{D} \rightarrow Y$ is A-proper, $C: \bar{D} \rightarrow Y$ compact, $f \notin T(\partial D)$ and $\|C x\|<\|T x-f\|$ for all $x \in \partial D$, then $\operatorname{Deg}(T, D, f)=$ $\operatorname{Deg}(T+C, D, f)$. In particular, $T x+C x=f$ has a solution in $D$ if $\operatorname{Deg}(T, D, f) \neq\{0\}$.

Proposition 4.1E has been extended in [42] to the following.

Proposition 4.1F. Let $T: \bar{D} \rightarrow Y$ be A-proper and $f \notin T(\partial D)$. Then there exists $c>0$ such that if $W: \bar{D} \rightarrow Y$ is $A$-proper, $f \notin W(\partial D)$ and $\| W x-$ $T x \| \leqq c$ for $x \in \partial D$, then $\operatorname{Deg}(T, D, f)=\operatorname{Deg}(W, D, f)$.

Using Proposition 1.1C one can easily show the following

Proposition 4.1G. If $T: \bar{D} \rightarrow Y$ is a continuous A-proper map and $f_{0}$ and $f_{1}$ belong to the same component of $X-T(\partial D)$, then $\operatorname{Deg}\left(T, D, f_{0}\right)=$ $\operatorname{Deg}\left(T, D, f_{1}\right)$.

The second and technically more delicate part of the generalized degree theory for $A$-proper mappings is the following theorem obtained in [25] for projectional schemes and in [24] for general schemes which gives a general sufficient condition for $\operatorname{Deg}(T, D, f)$ to be single-valued.

TheOREM 4.1H. Let $D$ be a bounded open subset of $X, T: \bar{D} \rightarrow Y$ a continuous A-proper mapping and $f \notin T(\partial D)$. Suppose that $T=A+C$, where $C: \bar{D} \rightarrow Y$ is compact and $A$ maps $D$ homeomorphically onto an open subset $A(D)$ of $Y$, carrying $\bar{D}$ homeomorphically onto $A(\bar{D})$. Let $T_{n}=$ $A_{n}+C_{n}: \bar{D}_{n} \rightarrow Y_{n}$ and suppose that $A_{n}$ is an orientation-preserving homeomorphism of $D_{n}$ into $Y_{n}$ such that

$$
\left\|A_{n}(x)-A_{n}(y)\right\| \geqq \alpha(\|x-y\|) \text { for all } x, y \in \bar{D}_{n} \text { and all } n,
$$

where $\alpha(t)$ is a continuous strictly increasing function of $R^{+}$into $R^{+}$with $\alpha(0)=0$.

Then there exists $n_{0} \geqq 1$ such that for $n \geqq n_{0}$,

$$
\operatorname{deg}\left(T_{n}, D_{n}, Q_{n} f\right)=\operatorname{deg}_{L S}\left(I+C A^{-1}, A(D), f\right) .
$$

In particular, $\operatorname{Deg}(T, D, f)=\left\{\operatorname{deg}_{L S}\left(I+C A^{-1}, A(D), f\right)\right\}$.

In view of the results outlined in Chapters 1 and 3, an immediate consequence is the following corollary of Theorem $4.1 \mathrm{H}$.

Corollary 4.1I. Suppose $Y=X$ and $X$ is $a \Pi_{1}$-space. Then the conclusion of Theorem $4.1 \mathrm{H}$ holds if either (I1) or (I2) is satisfied:

(I1) $A=I-S$ with $S$ a contraction on $X$. 
(I2) $A$ is strongly accretive (i.e. strongly J-monotone) on $X$ and either $X^{*}$ is uniformly convex or $A$ is uniformly continuous on bounded subsets of $X$.

Remark 4.1-2. It follows from Corollary $4.1 \mathrm{I}$ that when $X$ is a $\Pi_{1^{-}}$ space, then $\operatorname{Deg}(A+C, D, f)$ coincides with the Leray and Schauder degree $\operatorname{deg}_{L S}(I+C, D, f)$ when $A=I$ and with the Browder and Nussbaum degree when $A=I-S$.

It was shown by Webb [156] that if $F: \bar{D} \rightarrow X$ is ball-condensing, then $T=I-F$ is $A$-proper and in that case if $f \notin T(\partial D)$, then $\operatorname{Deg}(T, D, f)$ is single-valued and coincides with Sadovsky's degree $\operatorname{deg}_{x}(T, D, f)$. The original version of the above result was proved by Nussbaum [93] for $F$ such that $\gamma(F(Q)) \leqq k \gamma(Q)$ and $\chi(F(Q)) \leqq k \chi_{D}(Q)$ with $Q \subset D$ and $k<1$.

It was noted in [24] that if $F: \bar{D} \rightarrow X$ is $P_{1}$-compact, then for the homotopy $H_{t}(x): \bar{D} \times[0,1] \rightarrow X$ given by $H_{t}(x)=(1-t)(I-F) x+t x$ we deduce from Theorem 4.1B the fixed point theorem (see Corollary 1.2H) obtained in [129] without the use of the generalized degree.

THEOREM 4.1J. If $D$ is a bounded open subset of $X$ with $0 \in D$ and $F$ : $\bar{D} \rightarrow X$ is a $P_{1^{-}}$compact mapping which satisfies condition $\left(\pi_{1}^{<}\right)$on $\partial D$, then $\operatorname{Deg}(I-F, D, 0)=\{1\}$ and, in particular, $F$ has a fixed point in $D$.

The proof of Theorem 4.1J is based on the fact that the $A$-proper map $I-F$ is homotopic to the $A$-proper map $I$. In general, the identity mapping $I$ plays an essential role in the homotopy theory for various degrees for mappings acting in $X$. The next theorem established by the writer in [117] shows that when $X$ is reflexive, then the normalized duality mapping $J: X \rightarrow X^{*}$ can play a similar role for the generalized degree theory for mappings acting from subsets of $X$ into $X^{*}$.

Theorem 4.1K. Let $X$ be a reflexive $\Pi_{1}$-space with $X$ and $X^{*}$ having Property $(\mathrm{H})$. Then the normalized duality mapping $J: X \rightarrow X^{*}$ is continuous, odd and A-proper. Furthermore, for each given $r>0$ and for any fixed $w$ in $X^{*}$ with $J_{w}(x)=J(x)-w \neq 0$ for $x \in \partial B(0, r), \operatorname{Deg}\left(J_{w}, B(0, r), 0\right)$ is well defined, and

$$
\operatorname{Deg}\left(J_{w}, B(0, r), 0\right) \neq\{0\} \quad \text { if }\|w\|<r
$$

and

$$
\operatorname{Deg}\left(J_{w}, B(0, r), 0\right)=\{0\} \quad \text { if }\|w\|>r .
$$

We add in passing that an immediate consequence of Theorem $4.1 \mathrm{~K}$ is that $J$ is bijective, a fact proved earlier by Browder by different arguments. We will make some applications in the next sections.

For the sake of completeness we finish this section with the following remarks which will indicate further important and useful developments 
in the generalized degree theory for $A$-proper mappings and their uniform limits. We do not go into details in these remarks since these results lie outside the scope of our paper but an interested reader should consult the original papers.

REMARK 4.1-3. Using filters, ultrafilters and other concepts and results from nonstandard analysis developed by A. Robinson, Wong [158], [159] modified the definition of the generalized degree for a continuous $A$-proper map $T: \bar{D} \subseteq X \rightarrow Y$ given in [24], [25] to obtain again a function $\operatorname{Deg}(T, D, f)=\left\{\operatorname{deg}\left(T_{n}, D_{n}, Q_{n} f\right)\right\}$ whose values are sequences of integers in which two degrees are equal if they differ by at most a finite number of integers. His definition allowed him to obtain Theorem 4.1B with equality in the "sum formula" (B4) and thus he was able to define a fixed point index for a $P$-compact mapping and compute it in the case of differentiable mappings. Some further results were obtained in [160].

REMARK 4.1-4. Browder [16] and Fitzpatrick [38] independently extended the notion of generalized topological degree to mappings $T$ which are uniform limits on bounded sets of suitable sequences of $A$ proper mappings. They showed that under suitable conditions on the mappings their degrees have properties analogous to those of Theorem 4.1B and indicated applications to existence theorems for certain classes of equations.

A discussion of the generalized degree for $A$-proper mappings $T: \bar{D} \subset$ $X \rightarrow Y$ along the lines mentioned in Remark 4.1-1 (with $\bar{D}$ and $\partial D$ not necessarily the closure and the boundary of $D$ in $X$ ) was recently given by Petry [100]. The interesting feature of the paper is that it provides some useful sufficient conditions for a map $T: \bar{D} \subset H \rightarrow H$ to be $A$-proper with respect to $\Gamma_{0}$. In [97] it was shown that the notion of the multiplicity as defined by Cronin [29] can be incorporated into the notion of the generalized degree for a suitable $A$-proper mapping. For fixed point index results see Stuart [144].

4.2. Applications to some fixed point and mapping theorems. This section is devoted to the application of the generalized degree to fixed point and mapping theorems involving $A$-proper mappings $T: D \subset X \rightarrow Y$ defined on proper subsets of $X$. We are primarily concerned with three types of results: antipodes theorems, invariance of domain theorems, and structure of solution sets theorems.

REMARK 4.2-1. Before we state some applications of the generalized degree given by Definition 4.1A we should point out that, unlike other topological degrees, it embodies in its very structure a constructive aspect when applied to the solvability of a given equation $T x=f$ for $x$ in $D$. This is obviously true when $\operatorname{Deg}(T, D, f)$ is single-valued but it also happens in more general situations. Thus, for example, if $0 \notin \operatorname{Deg}(T, D, f)$, 
then there exists an integer $n_{0} \geqq 1$ such that $\operatorname{deg}\left(T_{n}, D_{n}, Q_{n} f\right) \neq 0$ for each $n \geqq n_{0}$. Hence, for each $n \geqq n_{0}$, there exists an element $x_{n} \in D_{n}$ such that $T_{n}\left(x_{n}\right)=Q_{n} f$. Since $Q_{n} f \rightarrow f$, the $A$-properness of $T$ implies the existence of a subsequence $\left\{x_{n_{j}}\right\}$ of $\left\{x_{n}\right\}$ and an element $x_{0}$ in $D$ such that $x_{n_{j}} \rightarrow x_{0}$ as $j \rightarrow \infty$ and $T\left(x_{0}\right)=f$, and in some cases (see Theorem 4.3G in $\S 4.3$ ) we can even assert that $x_{n} \rightarrow x_{0}$ as $n \rightarrow \infty$. This is, of course, more than just the assertion that $T x=f$ has a solution in $D$ if $0 \notin \operatorname{Deg}(T, D, f)$.

We know that, in general, a convex linear combination of two $A$-proper mappings is not $A$-proper. In view of this fact, the following definition will be convenient.

Definition 4.2A. Let $D$ be an open subset of $X$ which is symmetric about $0 \in D$. If $T: \bar{D} \rightarrow Y$ is a map such that $H: \bar{D} \times[0,1] \rightarrow Y$, given by

$$
H_{t}(x)=\frac{1}{1+t} T(x)-\frac{t}{1+t} T(-x)
$$

for $x \in \bar{D}$ and $t \in[0,1]$, is $A$-proper for each $t \in[0,1]$, then $T$ is said to be of convex A-proper type.

It follows from the results of Browder [19] that if $X$ is reflexive, if $D$ is also convex, and if $T: \bar{D} \rightarrow X^{*}$ bounded, continuous, and satisfies condition $\left(\mathrm{S}_{+}\right)$, then $T$ is of convex $A$-proper type. The paper [119], which extends the results of [19], [130], shows that if $X$ is reflexive, then $T: \bar{D} \rightarrow Y$ is of convex $A$-proper type if $T$ is of modified type $\left(\mathrm{S}_{+}\right)$and, in particular, if $T$ satisfies any one of conditions $(\mathrm{J} 2),(\mathrm{J} 3)$, or $(\mathrm{J} 4)$ of Theorem $3.1 \mathrm{~J}$ and Remark 3.1-1. Other maps which are of convex $A$-proper type will be mentioned in Chapter 5.

By using the generalized degree theory and Remark 4.2-1, it is not hard to establish (see [119]) the following results which include some basic classical theorems and indicate the importance of finding conditions for a given mapping $T$ to be of convex $A$-proper type. We start with the following antipodes theorem.

THEOREM 4.2B. Let $D$ be a bounded open subset of $X$ which is symmetric about $0 \in D$. If $T: \bar{D} \rightarrow Y$ is a bounded mapping of convex A-proper type such that

$$
T x \neq \lambda T(-x) \text { for } x \in \partial D \text { and } \lambda \in[0,1],
$$

then there exists $x_{0} \in D$ such that $T x_{0}=0$. Furthermore, there exists $n_{0} \geqq 1$ such that for each $n \geqq n_{0}$

$$
T_{n}(x)=0 \quad\left(x \in D_{n}\right)
$$

has a solution $x_{n} \in D_{n}$ and $x_{n_{j}} \rightarrow x_{0}$ for some subsequence of $\left\{x_{n}\right\}$, that is, 
the equation

$$
T x=0
$$

is feebly approximation-solvable in $D$, and strongly approximation-solvable if $x_{0}$ is unique.

Theorem 4.2B implies the validity of the following two corollaries for which it is not hard to verify that $T$ is of convex $A$-proper type. The first includes the well-known (see [67]) classical antipodes theorem for a compact map.

Corollary 4.2C. If $D \subset X$ is as in Theorem 4.2B and $F: \bar{D} \rightarrow X$ is a $k$-ball-contractive with $k<1$ and, in particular, compact, and if $T=I-F$ satisfies (4.2-1), then for $T=I-F$ the conclusions of Theorem 4.2B hold.

Corollary 4.2D. Let $X$ be a reflexive $\Pi_{1}$-space, $D=B(0, r), C: \bar{D} \rightarrow X$ compact and $S: \bar{D} \rightarrow X$ contractive. If either $S$ is weakly continuous or $X^{*}$ is strictly convex and a duality map $J: X \rightarrow X^{*}$ is weakly continuous, then the conclusions of Theorem 4.2B hold for $T=S+C-I$.

We note that the existence part of Corollary 4.2D can be obtained without any additional assumption on $X$ or $S$ as a corollary of an antipodes theorem for $k$-set-contractions $F: \bar{D} \rightarrow X$ with $k<1$ obtained later in [93] but its constructive aspect would be lost. Another consequence of Theorem 4.2B is the following result of the author [119].

Theorem 4.2E. Let $D \subset X$ be as in Theorem 4.2B and $T: \bar{D} \rightarrow Y$ bounded and of convex A-proper type. Suppose that either (E1) or (E2) holds, where

(E1) There is an odd $A: \bar{D} \rightarrow Y$ such that $\|T x-A x\|<\|A x\|$ for $x \in \partial D$;

(E2) $T x \neq 0$ and $T x /\|T x\| \neq T(-x) /\|T(-x)\|$ for $x \in \partial D$.

Then in either case equation (4.2-3) is feebly approximation-solvable in $D$.

The proof of Theorem 4.2E consists in showing that (E1) and (E2) imply the inequality (4.2-1). If $Y=X, D=B(0, r), T=I-C$ and $A=I-\lambda L$, then Theorem 4.2E, under condition (E1), implies the following Leray and Schauder result (see [67]).

Corollary 4.2F. If $D=B(0, r), C: \bar{D} \rightarrow X$ is compact and there exists a compact linear map $L: X \rightarrow X$ and $\lambda \neq 0$ such that $\|C x-L x\|<\|x-\lambda L x\|$ for $x \in \partial D$, then $C$ has a fixed point in $D$.

We complete this part of the section with another consequence of Theorem 4.2B for maps satisfying condition $\left(\mathrm{S}_{+}\right)$and modified condition $\left(\mathrm{S}_{+}\right)$ as well as for those satisfying the conditions of Theorem 3.1J. (See Theorem 7 in [119].) 
THEOREM 4.2G. Let $X$ be reflexive and $D$ a bounded, open and convex subset of $X$ which is symmetric about $0 \in D$. Let $K$ be a mapping of $X$ into $Y^{*}$, which satisfies the conditions of Theorem 3.1J and Remark 3.1-1, and let $T: \bar{D} \rightarrow Y$ be a bounded continuous mapping such that (4.2-1) holds. Suppose further that $T$ satisfies any one of the following conditions:

(G1) T satisfies (J4) of Remark 3.1-1; (G2) T satisfies (J3) of Theorem $3.1 \mathrm{~J}$; (G3) $T$ satisfies the modified condition $\left(\mathrm{S}_{+}\right)$.

Then equation (4.2-3) is feebly approximation-solvable in $D$ (in particular, equation (4.2-3) has a solution in $D)$.

RemarK 4.2-2. For the case where $Y=X^{*}$ and $K=I$, Theorem $4.2 \mathrm{G}$ was proved in [130] for the case when $T$ satisfies conditions (E2) and (J3) under stronger conditions on $T$ and $f$, and in [19] for the case when $T$ is bounded, continuous and of type $\left(S_{+}\right)$and satisfies condition (E2). Finally we remark that Theorem 1 in Nečas [92] follows directly from Theorem 4.1B when $\left(X, X^{*}\right)$ has a projectionally complete scheme or from Theorem 1 in [24] when $X$ is separable.

Invariance of domain theorems. In this section we state the basic invariance of domain theorem for locally $A$-proper mappings $T: D \subset X \rightarrow Y$ obtained by the writer in [117] and deduce from it some classical as well as some recent invariance of domain theorems for compact and noncompact translations.

Suppose that (P) denotes a certain property. We say that $T: D \subset X \rightarrow Y$ has a local property $(\mathrm{P})$ if to each $x_{0}$ there corresponds a neighborhood $B\left(x_{0}, r\right)$ with $\bar{B}\left(x_{0}, r\right) \subset D$ such that $T: \bar{B}\left(x_{0}, r\right) \rightarrow Y$ has property (P). We first state the following lemma which, in addition to its independent interest, plays an essential role in the proof of the invariance of domain theorem for locally $A$-proper mappings.

LEMMA 4.2H. If $T: \bar{B}\left(x_{0}, r\right) \subset X \rightarrow Y$ is a continuous A-proper mapping such that $T(x) \neq T\left(x_{0}\right)$ for $x \in \partial B\left(x_{0}, r\right)$ and $\operatorname{Deg}\left(T, B\left(x_{0}, r\right), T\left(x_{0}\right)\right) \neq\{0\}$, then there exists a neighborhood $B\left(T\left(x_{0}\right), s\right) \subset Y$ consisting entirely of points which are images of points of $\bar{B}\left(x_{0}, r\right)$ under $T$.

Theorem 4.1B and Lemma 4.2H imply the validity of the following invariance of domain theorem.

Theorem 4.2I. If $D \subset X$ is open, $T: D \rightarrow Y$ is a continuous locally $A$ proper mapping such that $\operatorname{Deg}\left(T, B\left(x_{0}, r\right), T\left(x_{0}\right)\right) \neq\{0\}$ for each $x_{0}$ in $D$ and some $r>0$, then $T(D)$ is an open set in $Y$.

For Theorem 4.2I to be useful in applications we need some simple conditions which would imply $\operatorname{Deg}\left(T, B, T\left(x_{0}\right)\right) \neq\{0\}$. It is easy to see that the next theorem serves that purpose. 
TheOREM 4.2J. Suppose $D \subset X$ is open, $T: D \rightarrow Y$ is continuous and to each $x_{0} \in D$ there corresponds $\bar{B}\left(x_{0}, r\right) \subset D$ and a continuous map $H_{t}(\cdot)$ : $\bar{B}\left(x_{0}, r\right) \times[0,1] \rightarrow Y$ such that

(J1) $H_{t}(\cdot)$ satisfies $\left(\mathrm{B}^{\prime}\right)$ and, in particular, (B3) of Theorem 4.1B.

(J2) $H_{0}(x)=T(x)$ for $x \in \bar{B}\left(x_{0}, r\right)$ and $H_{t}(x) \neq T\left(x_{0}\right)$ for $x \in \partial D$ and $t \in[0,1]$.

If $\operatorname{Deg}\left(H_{1}, B\left(x_{0}, r\right), T\left(x_{0}\right)\right) \neq\{0\}$, then $T(D)$ is open.

In view of Theorem 4.1B, Theorem 4.2I implies the validity of the following result for local $\varepsilon$-mappings established by Granas [48] as a generalization of the invariance of domain theorems of Schauder [137] and Leray [78]. $T: D \rightarrow Y$ is called a local $\varepsilon$-mapping if to each $x_{0} \in D$ there corresponds $B\left(x_{0}, \varepsilon_{x_{0}}\right)$ with $\bar{B}\left(x_{0}, \varepsilon_{x_{0}}\right) \subset D$ such that $T x_{1}=T x_{2}$ implies that $\left\|x_{1}-x_{2}\right\|<\varepsilon_{x_{0}}$ for any $x_{1}, x_{2} \in B\left(x_{0}, \varepsilon_{x_{0}}\right)$.

Corollary 4.2K. If $D \subset X$ is open and $T=I+C: D \rightarrow X$ is a local $\varepsilon$ mapping with $C: D \rightarrow X$ locally compact, then $T(D)$ is open in $X$.

To see how Theorems 4.1B and 4.2J are used, let $x_{0}$ be any point in $D$. Without loss of generality assume that $x_{0}=0$ and $T(0)=0$. For some $r>0$ define $H_{t}(\cdot): \bar{B}(0, r) \times[0,1] \rightarrow X$ by $H_{t}(x)=x+C(x /(1+t))-C(-t x /(1+t))$. One shows that $H_{t}(\cdot)$ satisfies condition $\left(\mathrm{B}^{\prime}\right)$ with $H_{t}(x) \neq 0$ for $x$ in $\partial B(0, r)$ and $t \in[0,1]$. Thus, $\operatorname{Deg}\left(H_{t}, B(0, r), 0\right)$ is independent of $t \in[0,1]$. Since $H_{1}$ is odd on $\bar{B}(0, r), \operatorname{Deg}(T, B(0, r), 0) \neq\{0\}$. Consequently, by Theorem $4.2 \mathrm{~J}, T(D)$ is open.

Using the same arguments one gets the following generalization of Corollary $4.2 \mathrm{~K}$ which contains an invariance of domain theorem obtained in [156].

Corollary 4.2L. Let $X$ be a $\Pi_{1}$-space. If $D \subset X$ is open and $T=I-$ $F: D \rightarrow X$ is a local $\varepsilon$-map with $F: D \rightarrow X$ locally $k$-ball-contractive with $k<1$, then $T(D)$ is open.

In view of the results of Nussbaum [93], Theorems 4.1B and 4.2J imply the validity of the following corollary which includes the result of Schauder in the case when $X$ is reflexive, $S$ weakly continuous, $C$ is completely continuous and $T=I-S-C$ is one-to-one.

CoROllaRY 4.2M. Let $X$ be $a \Pi_{1}$-space. If $D \subset X$ is open and $T=I-S-$ $C: D \rightarrow X$ a local $\varepsilon$-map with $S: D \rightarrow X$ and $C: D \rightarrow X$ locally contractive and locally compact, respectively, then $T(D)$ is open.

Now we indicate some other classes of mappings to which Theorems 4.2I and 4.2J are applicable (see [117]).

THEOREM 4.2N. If $D \subset X$ is open and $T_{\lambda}=A+\lambda I: D \rightarrow X$ is both locally one-to-one and $A$-proper for all $\lambda \geqq 0$, then $T_{\lambda}(D)$ is open for each $\lambda \geqq 0$. 
As a consequence of Theorem $4.2 \mathrm{~N}$ we deduce the following corollary which contains the Browder and Minty result for locally strongly monotone mappings.

Corollary 4.2O. Let $H$ be a Hilbert space, $D \subset H$ open and $A$ a continuous locally monotone map of $D$ into $H$ which satisfies the modified condition (S). If $A$ is locally one-to-one, then $T_{\lambda}(D)$ is open for each $\lambda \geqq 0$.

In view of Theorem $4.1 \mathrm{~K}$ and (B3) of Theorem 4.1B, Corollary 4.20 admits the following extension obtained [117] (see [21] for an analogous result but under different conditions).

Theorem 4.2P. Let $X$ be reflexive with $X$ and $X^{*}$ having Property (H). If $T$ is a locally monotone and locally one-to-one map of an open set $D \subset X$ into $X^{*}$ which satisfies condition (S) on $D$, then $T(D)$ is open in $X^{*}$.

Using Theorem $4.1 \mathrm{~K}$ and the generalized degree theory for uniform limits of $A$-proper mappings, Theorem $4.2 \mathrm{P}$ has been further extended in [38].

Structure of solution sets. It was noted in $\$ 1.2$ that the result in [71], [30], [153] concerning the structure of fixed point sets has been extended by Fitzpatrick [39], [42] to equations involving $A$-proper mappings and their uniform limits. For $A$-proper mappings this extension can be stated in the following form.

THEOREM 4.2Q. Let $D \subset X$ be open and bounded and $T: \bar{D} \rightarrow Y$ A-proper and proper. Suppose there exists a sequence of $A$-proper mappings $T^{k}: \bar{D} \rightarrow Y$ such that

(R1) $\sup \left\{\left\|T^{k}(x)-T(x)\right\| \mid x \in \bar{D}\right\}=\delta_{k} \rightarrow 0$ as $k \rightarrow \infty$,

(R2) $T^{k}$ is one-to-one when restricted to $\left(T^{k}\right)^{-1}\left(B\left(0, \delta_{k}\right)\right)$. Then if $\operatorname{Deg}(T, D, 0) \neq\{0\}, T^{-1}(0)$ is a continuum.

The proof of Theorem 4.2Q was based on Theorem 4.1B and Proposition 4.1F. Deimling [30] had earlier obtained a result similar to Theorem $4.2 Q$ for continuous $A$-proper mappings $T: D \subset X \rightarrow X$ but his proof, unlike that of [42], depended essentially upon the fact that $T$ and $T^{k}$ were $A$ proper. It was already noted in [30] that the result of [71] follows from Theorem 4.2Q when $Y=X, T=I-C$ and $T^{k}=I-C^{k}$ with $C$ and $C^{k}$ compact. In [42] Fitzpatrick deduced as corollaries of Theorem $4.2 Q$ the known results obtained in [71], [30], [153] as well as some new ones. In addition to those already mentioned we state as illustrations two further corollaries, the first of which is due to Vidossich [153].

COROLlaRY 4.2R. Let $C: \bar{B}\left(x_{0}, r\right) \subset X \rightarrow X$ be compact and $T=I-$ $C: \bar{B} \rightarrow X$ be such that $y_{0} \notin T(\partial B)$ where $y_{0}=T\left(x_{0}\right)$. Suppose there exists a sequence of compact mappings $C^{k}: \bar{B} \rightarrow X$ such that $T^{k}=I-C^{k}: X \rightarrow X$ is a 
homeomorphism for each $k$. If $\left\{T^{k}\right\}$ converges uniformly to $T$, then $T^{-1}\left(y_{0}\right)$ is a continuum.

Corollary 4.2S. Let $D \subset X$ be open and bounded, $T: \bar{D} \rightarrow X P_{1^{-}}$ compact with $T(\partial D)$ bounded and $I-T$ proper and J-monotone. Then if the fixed point set $F(T) \neq \varnothing$ and $F(T) \cap \partial D=\varnothing, F(T)$ is a continuum.

4.3. Fréchet differentiable and analytic type A-proper mappings. In the first part of this section we outline some basic results obtained in [129], [158], [39] for $F$-differentiable $P$-compact and $A$-proper mappings. In the second part we state the results obtained in [133] for $A$-proper mappings of the analytic type. We conclude the section with some new results involving the strong approximation-solvability of the equation $T x=f$ $(x \in D)$ having an isolated solution $x_{0} \in D$ with an $A$-proper $F$-derivative $T_{x_{0}}^{\prime}$ at $x_{0} \in D$.

Recall first that if $D \subset X$ is open and $T: D \rightarrow Y$, then $T$ is said to be $F$ differentiable at $x \in D$ if there exists $T_{x}^{\prime} \in L(X, Y)$, called the $F$-derivative of $T$ at $x$, such that for each $y$ in $D$ one has

$T x-T y=T_{x}^{\prime}(x-y)+R(x, y) \quad$ with $\frac{\|R(x, y)\|}{\|x-y\|} \rightarrow 0$ as $\|x-y\| \rightarrow 0$.

It is known that if $C: D \subset X \rightarrow X$ is compact and $F$-differentiable at $x \in D$, then $C_{x}^{\prime}$ is also compact. It was shown in [161] that the converse is false. However, Vainberg has shown in [147] that if $C: X \rightarrow Y$ is $F$-differentiable at each $x \in X, C_{x}^{\prime}$ is compact and $C^{\prime}: X \rightarrow L(X, X)$ defined by $x \rightarrow C_{x}^{\prime}$ is compact, then $C$ is compact. It was noted in [42] that an example of [161] can be used to show that $T: X \rightarrow X$ can have an $A$-proper (in fact, $P$ compact) $F$-derivative $T_{x}^{\prime}$ at each $x \in X$ without itself being $A$-proper.

EXAMPLE 4.3-1. Let $X=l_{2}$ and $C(x)=\sum_{i=1}^{\infty}\left(\phi_{i}, x\right)^{2} \phi_{i}$ for $x \in l_{2}$, where $\left\{\phi_{i}\right\}$ is the natural basis for $l_{2}$. Then $T=I-C$ is not $A$-proper since $T \phi_{i}=0$ and so $P_{i} T \phi_{i}=0 \rightarrow 0$ as $i \rightarrow \infty$. But $\left\{\phi_{i}\right\}$ has no convergent subsequence. Since $C_{x}^{\prime}(y)=\sum_{i=1}^{\infty}\left(x, \phi_{i}\right)\left(y, \phi_{i}\right) \phi_{i}$ for each $y$ in $l_{2}, C_{x}^{\prime}$ is compact for each $x \in l_{2}$ and $T_{x}^{\prime}=I-C_{x}^{\prime}$ is $A$-proper.

An analogue of Vainberg's result was obtained in Petryshyn and Tucker [129], for $P$-compact $F: X \rightarrow X$ under strengthened continuity conditions on $F_{x}^{\prime}$.

TheOREM 4.3A. Let $X$ be reflexive and let $F: X \rightarrow X$ be $F$-differentiable at each $x \in X$. Suppose that $F^{\prime}: X \rightarrow L(X, X)$ is completely continuous (i.e., $F_{x_{j}}^{\prime} \rightarrow F_{x}^{\prime}$ in $L(X, X)$ if $x_{j} \rightarrow x$ in $\left.X\right)$. Then $F_{x}^{\prime}$ is $P$-compact at each $x$ in $X$ if and only if $F$ is $P$-compact. 
Using the technique of [129], it was shown in [39] that an analogous result holds for an $F$-differentiable $A$-proper mapping $T: X \rightarrow Y$ with $X$ reflexive under the following slightly weaker continuity condition :

$$
T_{y_{n}}^{\prime}\left(x-z_{n}\right)-T_{x}^{\prime}\left(x-z_{n}\right) \rightarrow 0 \quad \text { whenever } y_{n} \rightarrow x \text { and } z_{n} \rightarrow x \text { in } X .
$$

It was shown in [129] that if $A: X \rightarrow X$ is a linear $P$-compact operator and $\lambda>0$ is not an eigenvalue of $A$, then $\lambda I-A$ is bijective. This fact was then used to show that if $F$ has a fixed point $x_{0}$ in $X$ and $F$ is $F$-differentiable at $x_{0}$ with $I-F_{x_{0}}^{\prime}$ injective, then $x_{0}$ is an isolated fixed point of $F$. Using his modified version of the generalized degree, in [159] Wong extended to $P$-compact mappings with $P$-compact $F$-derivatives the theorem of Leray and Schauder [80] which states that if $C: X \rightarrow X$ is an $F$-differentiable compact mapping and $x_{0} \in X$ is a fixed point of $C$ such that $T_{x_{0}}^{\prime}=I-C_{x_{0}}^{\prime}$ is injective, then there exists a neighborhood $B\left(x_{0}, r\right)$ such that $\operatorname{deg}_{L S}\left(T, B\left(x_{0}, r\right), T\left(x_{0}\right)\right)=\operatorname{deg}_{L S}\left(T_{x_{0}}^{\prime}, B(0, r), 0\right)$.

In [42] Fitzpatrick extended the results of [80], [159] to the generalized degree for $A$-proper mappings as given by Definition $4.1 \mathrm{~A}$ by proving the following basic theorem.

Theorem 4.3B. Suppose $D \subset X$ is open and bounded and $T: \bar{D} \rightarrow Y$ is A-proper with F-derivative $T_{x_{0}}^{\prime}$ at $x_{0} \in D$ such that $T_{x_{0}}^{\prime}$ is A-proper and injective. Then $x_{0}$ is an isolated solution of $T x=T\left(x_{0}\right)(x \in D)$ and there exist a neighborhood $\bar{B}\left(x_{0}, r\right) \subset D$ such that

$$
\operatorname{Deg}\left(T, B\left(x_{0}, r\right), T\left(x_{0}\right)\right)=\operatorname{Deg}\left(T_{x_{0}}^{\prime}, B\left(x_{0}, r\right), T_{x_{0}}^{\prime}\left(x_{0}\right)\right) .
$$

Combining Proposition 4.1D and Theorem 4.3B the following generalization of the classical result has been obtained in [42].

COROLLARY 4.3C. Under the conditions of Theorem 4.3B,

$$
\operatorname{Deg}\left(T, B\left(x_{0}, r\right), T\left(x_{0}\right)\right)=\operatorname{Deg}\left(T_{x_{0}}^{\prime}, B(0, r), 0\right) \text {. }
$$

Let us remark that in virtue of Proposition 1.1C and the first part of Theorem 4.3B or the corresponding result for $P$-compact mappings it follows (see [42], [159]) that if $T: \bar{D} \subset X \rightarrow Y$ is a continuous $A$-proper mapping, if $T\left(x_{0}\right)=f$ for some $x_{0} \in D$ and if $T_{x_{0}}^{\prime}$ exists, is $A$-proper and injective, then there are at most a finite number of solutions in $D$ of the equation $T x=f$.

$A$-proper mappings of analytic type. If $Z$ is a complex Banach space and $U$ an open subset of $Z$, then a mapping $f$ of $U$ into $Z$ is said to be complex analytic if for each pair $x$ and $y$ of $Z$ with $x$ in $U$, the function $f(x+\xi y)$ of a single complex variable $\xi$ is analytic in $\xi$ on some neighborhood of the origin. In [27] Cronin used the Leray and Schauder degree to establish existence and uniqueness theorems for a class of compact complex analytic 
operators. In [17] Browder introduced and studied the notion of a mapping of analytic type acting in a real Banach space and showed that a complex analytic mapping, if considered as operating in the real space obtained from the complex space by ignoring the complex structure, is of analytic type. This allowed him to extend the results of [27], [139] to a wider class of mappings of analytic type.

Using the generalized degree developed in [24], [25], Potter [133] extended Browder's results on analytic type compact operators to analytic type $A$-proper mappings. An interesting consequence of these results in [133] is the uniqueness theorem for $k$-ball-contractions with $k<1$ acting in $\Pi_{1}$-space $X$.

Definition 4.3D. Let $X$ be a real $\Pi_{1}$-space, $U \subset X$ open and $T$ a continuous map of $U$ into $X$. Then $T$ is said to be of analytic type with respect to $\Gamma_{0}$ if the mappings $T_{n}: U_{n} \subset X_{n} \rightarrow X_{n}$ are of analytic type in the sense of Browder (see [17; Definitions 1, 2 and 3]).

Using Browder's finite dimensional results from [17], Proposition 1.1C and Theorem 4.1B, the following results were obtained for $A$-proper mappings.

THEOREM 4.3E. Let $U \subset X$ be open, $D \subset U$ bounded and open with $\bar{D} \subset U$ and $T: U \rightarrow X$ a continuous map of analytic type which is A-proper on $\bar{D}$. Let $f \in X-T(\partial D)$. Then

(E1) $\operatorname{Deg}(T, D, f)$ contains no negative integers;

(E2) $0 \notin \operatorname{Deg}(T, D, f)$ if and only if $f \in T(D)$;

(E3) If $1 \in \operatorname{Deg}(T, D, f)$, then $T^{-1}(f) \cap D$ is connected;

(E4) If $T(D)$ contains a point of a given component $G$ of $X-T(\partial D)$, then $G \subset T(D)$.

Moreover, suppose that for each $f \in X-T(\partial D)$ the set $T^{-1}(f) \cap D$ is totally disconnected. Then for any $f \in X-T(\partial D)$ such that $1 \in \operatorname{Deg}(T, D, f)$, $T^{-1}(f) \cap D$ is a single point; and for the component $G$ of $X-T(\partial D)$ containing $f, T$ is a homeomorphism of $T^{-1}(G) \cap D$ onto $G$.

Let $Z$ be a complex $\Pi_{1}$-space and let $X$ be the real Banach space obtained from $Z$ by ignoring the complex structure. Obviously $X$ is a $\Pi_{1}$ space and if $F: D(F) \subset Z \rightarrow Z$ is $k$-ball-contractive, then it is $k$-ball-contractive when considered as operating in $X$. Hence, by a result of Webb [156], (see also Nussbaum [93]), $T=I-F$ is $A$-proper.

Using the above fact, the second part of Theorem $4.3 \mathrm{E}$ and certain results of Browder (see [19, Chapter 15]), the following uniqueness theorem has been obtained in [133].

Theorem 4.3F. Let $Z$ be a complex $\Pi_{1}$-space, $U \subset Z$ open and $D$ an open convex bounded subset of $U$ with $\bar{D} \subset U$. If $F: U \rightarrow Z$ is complex 
analytic and $k$-ball-contractive with $k<1$ as a map of $\bar{D}$ into $Z$ such that $F(\partial D) \subset D$ and $F x \neq x$ for $x \in \partial D$, then $F$ has a unique fixed point in $D$.

Strong approximation-solvability. In $\$ 3$ we outlined various results concerning the unique and strong approximation-solvability of the equation

$$
T(x)=f \quad(x \in D, f \in Y)
$$

involving an $A$-proper mapping $T: D \subset X \rightarrow Y$ under the assumption that equation (4.3-2) has at most one solution. Assuming that $T$ has an $A$ proper $F$-derivative at a solution $x_{0} \in D$ of (4.3-2) and using Corollaries 4.3C and 4.1C we strengthen here the approximation-solvability results for (4.3-2) without the uniqueness assumption. Thus we extend to equation (4.3-2) Theorems 3.1 and 3.2 of Krasnoselsky [67] (see also Vainikko [148]) which treat the case where $f=0, T=I-C$, and $C: D \rightarrow X$ is compact.

Recall first that $T$ is continuously $F$-differentiable at $x_{0} \in D$ if $T$ is $F$-differentiable in a neighborhood of $x_{0}$ in $D$ and $\left\|T_{x}^{\prime}-T_{x_{0}}^{\prime}\right\| \rightarrow 0$ as $x \rightarrow x_{0}$. In view of (4.3-1), this implies that

$$
\|R(x, y)\| /\|x-y\| \rightarrow 0 \text { as } x \rightarrow x_{0} \text { and } y \rightarrow x_{0} .
$$

THEOREM 4.3G. Let $D \subset X$ be open and $T: \bar{D} \rightarrow Y$ A-proper. Let $x_{0} \in D$ be a solution of equation (4.3-2) and suppose that $T$ is $F$-differentiable at $x_{0}$ with $T_{x_{0}}^{\prime}$ injective and A-proper. Then equation (4.3-2) is strongly approximation-solvable in $B\left(x_{0}, r\right) \subset D$ for some $r>0$, i.e., there exist $r>0$ and $n_{0} \geqq 1$ such that equation (4.3-2) has $x_{0}$ as its only solution in $\bar{B}\left(x_{0}, r\right) \subset D$ and the equation

$$
T_{n}(x)=Q_{n} f \quad\left(x \in B_{n}\left(x_{0}, r\right)=X_{n} \cap B\left(x_{0}, r\right)\right)
$$

has a solution $x_{n} \in B_{n}\left(x_{0}, r\right)$ for each $n \geqq n_{0}$ such that $x_{n} \rightarrow x_{0}$ as $n \rightarrow \infty$. Moreover, if we set $\gamma=\left\|\left(T_{x_{0}}^{\prime}\right)^{-1}\right\|$, then for any $\varepsilon \in(0, \gamma)$ there exists $n_{1}$ $\left(\geqq n_{0}\right)$ such that

$$
\left\|x_{n}-x_{0}\right\| \leqq(\gamma-\varepsilon)^{-1}\left\|T x_{n}-f\right\| \text { for } n \geqq n_{1} .
$$

If we also assume that $T$ is continuously $F$-differentiable at $x_{0}$, then there exists $n_{2}\left(\geqq n_{1}\right)$ such that equation (4.3-4) is uniquely solvable for $n>n_{2}$. Moreover, there is a constant $M$ which depends on $\left\|T_{x_{0}}^{\prime}\right\|, \gamma$ and $\beta=\sup \left\|Q_{n}\right\|$, such that

$$
\left\|x_{n}-x_{0}\right\| \leqq M\left\|x_{0}-P_{n} x_{0}\right\| \text { for all sufficiently large } n .
$$

Proof. We shall outline the proof. Note that, by Corollaries $4.3 \mathrm{C}$ and 4.1C, there exist $r>0$ and $n_{0} \geqq 1$ such that $x_{0}$ is the only solution of (4.3-2) in $\bar{B}\left(x_{0}, r\right)(\subset D)$ and $\operatorname{deg}\left(T_{n}, B_{n}\left(x_{0}, r\right), Q_{n} f\right) \neq 0$ for $n \geqq n_{0}$. Hence 
there exists $x_{n} \in B_{n}\left(x_{0}, r\right)$ such that $T_{n}\left(x_{n}\right)=Q_{n} f$ and so, by the $A$ properness of $T$ and the property of $B\left(x_{0}, r\right), x_{n} \rightarrow x_{0}$ as $n \rightarrow \infty$. In view of this, our conditions on $T_{x_{0}}^{\prime}$ and the equality

$$
f-T x_{n}-R\left(x_{0}, x_{0}-x_{n}\right)=T x_{0}-T x_{n}-R\left(x_{0}, x_{0}-x_{n}\right)=T_{x_{0}}^{\prime}\left(x_{0}-x_{n}\right),
$$

it follows from (4.3-1) and Theorem 2.1A that estimate (4.3-5) holds for some $n_{1}\left(\geqq n_{0}\right)$.

To prove the second part of Theorem 4.3G, note that if $\left\{z_{n}\right\} \subset B\left(x_{0}, r\right)$ is such that $z_{n} \rightarrow x_{0}$, then since $T$ is continuously $F$-differentiable at $x_{0}$ there exist $c>0$ and $n_{2}\left(\geqq n_{1}\right)$ such that

$$
\left\|Q_{n} T_{z_{n}}^{\prime}(x)\right\| \geqq c\|x\| \quad \text { for all } x \in X_{n} \text { and all } n \geqq n_{2} \text {. }
$$

From this and the fact that $\left\|R\left(P_{n} x_{0}, P_{n} x_{0}-x_{n}\right)\right\| /\left\|P_{n} x_{0}-x_{n}\right\| \rightarrow 0$ as $n \rightarrow \infty$, it follows that equation (4.3-4) is uniquely solvable in $B\left(x_{0}, r\right)$ for each $n \geqq n_{2}$. Setting $z_{n}=P_{n} x_{0}$ and using the fact that $Q_{n} T x_{n}=Q_{n} f$ with $T x_{0}=f$ and the equality

$$
\begin{aligned}
Q_{n} T_{z_{n}}^{\prime}\left(x_{n}-z_{n}\right) & =Q_{n} T x_{n}-Q_{n} T z_{n}-Q_{n} R\left(z_{n}, x_{n}-z_{n}\right) \\
& =Q_{n}\left\{T x_{0}-T z_{n}-R\left(z_{n}, x_{n}-z_{n}\right)\right\}
\end{aligned}
$$

we see that to any given $\varepsilon \in(0, c)$ there exists $n_{3}\left(\geqq n_{4}\right)$ such that $(c-\varepsilon)\left\|x_{n}-z_{n}\right\| \leq \beta\left\|T x_{0}-T z_{n}\right\|$ for $n \geq n_{3}$ where $\left\|Q_{n}\right\| \leq \beta$ for all $n$.

On the other hand, $\left\|T x_{0}-T z_{n}\right\| \leqq\left\|T_{x_{0}}^{\prime}\right\|\left\|z_{n}-x_{0}\right\|+\varepsilon_{1}\left\|z_{n}-x_{0}\right\|$ for $n \geqq n_{4}$ $\left(\geqq n_{3}\right.$ ) and any given $\varepsilon_{1}>0$. Combining the above inequalities one obtains the estimate (4.3-6). Q.E.D.

REMARK 4.3-1. Theorem 4.3G is applicable, in particular, to the case when $Y=X, f=0$ and $T=I-F$, where $F: \bar{D} \rightarrow X$ is $P_{1}$-compact and, in particular, ball-condensing or compact mapping. Thus, Theorems 3.1 and 3.2 in [67], as well as Theorem 7 in [108] for $\lambda=1$ (without the restricting assumption (23) used in [108]), are deducible as corollaries of Theorem 4.3G.

\section{Existence theorems and pseudo- $A$-proper type mappings}

In preceding sections we outlined the theory of $A$-proper maps. Among other results, we showed the intimate relation of $A$-properness to the question of the approximation-solvability of equations. Although the class of $A$-proper maps is quite large, there are existence theorems for operator equations (e.g. with weakly closed or with monotone type operators) for which the $A$-proper mapping theory is not directly applicable. This is not surprising since $A$-properness is connected with constructive solvability 
and one cannot expect this property of all equations for which existence of solutions can somehow be established.

However, it was noted in [118] that a closer look at $A$-proper mapping theory suggests that if one is primarily interested in its existential rather than its constructive aspect, then the same approach can still be used to obtain existence theorems for a much wider class of pseudo-A-proper maps and for uniform limits of $A$-proper and pseudo- $A$-proper maps. In this section we present three basic existence theorems for equations involving the above classes of mappings and then use these theorems to deduce, on the one hand, fixed point theorems for $G$-operators, $P_{1}$-compact, ball-condensing and weakly closed operators and, on the other hand, surjectivity theorems for operators of weakly closed, $K$-monotone and, in particular, of monotone type. As a result, we deduce from our general results a number of theorems obtained earlier by various authors and methods.

The price we pay for using these $A$-proper type methods to obtain existence theorems for such a general class of mappings $T: D \subseteq X \rightarrow Y$ is the requirement that the spaces $X$ and $Y$ have a projectionally complete scheme (or at least be separable since the latter case can be handled by using the more general schemes as, for example, in [24], [112], [115], [50]). We add that, as will be seen below, for many special classes of pseudo- $A$-proper mappings our approach provides also the possibility of obtaining solutions as weak limits of finite dimensional approximants.

5.1. Equations involving pseudo-A-proper mappings. Let $(X, Y)$ be a pair of real Banach spaces, $D$ a subset of $X, T$ a map of $D$ into $Y$ and $\Gamma=\left\{X_{n}, P_{n} ; Y_{n}, Q_{n}\right\}$ a projectionally complete scheme for $(X, Y)$ which allows us to associate with the equation

$$
T(x)=f \quad(x \in D, f \in Y)
$$

a sequence of approximate equations

$$
T_{n}(x)=Q_{n} f \quad\left(x \in D_{n}, T_{n}=\left.Q_{n} T\right|_{D_{n}}, Q_{n} f \in Y_{n}\right) .
$$

Definition 5.1A [118]. A mapping $T: D \subseteq X \rightarrow Y$ is said to be pseudo$A$-proper with respect to $\Gamma$ if $T_{n}: D_{n} \rightarrow Y_{n}$ is continuous and if $T$ satisfies condition (h): if $\left\{x_{n_{j}} \mid x_{n_{j}} \in D_{n_{j}}\right\}$ is any bounded sequence such that $T_{n_{j}}\left(x_{n_{j}}\right) \rightarrow g$ for some $g$ in $Y$, then there exists $x \in D$ such that $T x=g$.

Mappings satisfying condition (h) have been introduced in [114] and further studied in [118], [121] and in Wong [158]. As we shall see later for the case when $Y=X$ the concept of a pseudo- $A$-proper mapping is related to the notion of a $G$-operator introduced by de Figueiredo [36]. Unless stated otherwise, it is always assumed that the Banach spaces $(X, Y)$ 
have a projectionally complete scheme $\Gamma$ and to say that $T$ is pseudo$A$-proper means that it is so with respect to that particular scheme. In what follows $K$ will be a mapping (in general nonlinear) from $X$ to $Y^{*}$ such that $K(0)=0, K x \neq 0$ if $x \neq 0$ and (3.1-2) holds (i.e., $Q_{n}^{*} K x=K x$ for $x \in X_{n}$ ). The mapping $M_{n}$ will always be a linear isomorphism of $X_{n}$ onto $Y_{n}$ such that (3.1-4) holds (i.e. $\left(M_{n}(x), Q_{n}^{*} K(x)\right)>0$ for $x \in X_{n}$ with $x \neq 0$ ).

It turns out that the existence part of Theorem $3.1 \mathrm{~K}$ remains valid for pseudo- $A$-proper mappings (see the author's paper [121] for the proofs of most of the results mentioned in this section).

Theorem 5.1B. Let $K$ and $M_{n}$ satisfy (3.1-2) and (3.1-4) respectively.

(B1) If $D$ is a bounded open subset of $X$ with $0 \in D, T: \bar{D} \rightarrow Y$ pseudo- $A$ proper and $f$ an element in $Y$ such that

$$
(T x, K x) \geqq(f, K x) \text { for } x \in \partial D,
$$

then equation (5.1) has a solution in $\bar{D}$ for each $f$ satisfying (5.1-1).

(B2) If $T: X \rightarrow Y$ is pseudo-A-proper and $K$-coercive, then equation (5.1) has a solution $x$ in $X$ for each $f$ in $Y$.

The proof of Theorem 5.1B is based on the following known finite dimensional fixed point theorem [28].

LERAY-SCHAUDER THEOREM. Let $V$ be a finite dimensional Banach space, $G$ an open bounded subset of $V$ with $0 \in G$ and $A: G \rightarrow V$ a continuous mapping such that $A x \neq \lambda x$ for $x \in \partial G$ and $\lambda>1$, then $A$ has a fixed point in $\bar{G}$.

REMARK 5.1-1. Observe the qualitative difference between Theorems $3.1 \mathrm{~K}$ and $5.1 \mathrm{~B}$. Theorem $3.1 \mathrm{~K}$ yields essentially a constructive existence of solutions of equation (5.1) involving $A$-proper mappings while Theorem 5.1B yields only the existence of solutions of equation (5.1) involving pseudo- $A$-proper mappings. Nevertheless, as we shall see later, for many special classes of pseudo- $A$-proper mappings our approach provides also the possibility of obtaining solutions of equation (5.1) as weak limits of solutions $\left\{x_{n} \mid x_{n} \in D_{n}\right\}$ of equation (5.2) especially when the uniqueness is known.

In view of this it makes sense to introduce the following

Definition 5.1C. Equation (5.1) is said to be weakly approximationsolvable if equation (5.2) has a solution $x_{n} \in D_{n}$ for all sufficiently large $n$ such that $x_{n} \rightarrow x$ in $D$ and $T x=f$.

Special classes of pseudo-A-proper mappings. In what follows we consider some important examples of pseudo- $A$-proper mappings $T: D \subseteq X \rightarrow Y$ for which Theorem 5.1B is applicable. In the Historical Remarks we will indicate the relation of the results presented in various corollaries below 
to fixed point theorems and surjectivity theorems obtained by other authors for various special classes of mappings and usually by different arguments.

(a1) Weakly closed and weakly continuous mappings. Recall that $T: \bar{D} \rightarrow Y$ is said to be weakly closed if whenever $\left\{x_{n}\right\} \subset D$ is a sequence such that $x_{n} \rightarrow x$ in $X$ and $T x_{n} \rightarrow h$ in $Y$, then $x \in D$ and $T x=h$. It is not hard to see that if $X$ and $Y$ are reflexive, $K: X \rightarrow Y^{*}$ demicontinuous with $R(K)$ dense in $Y^{*}, T: \bar{D} \rightarrow Y$ bounded and weakly closed and (3.1-2) holds, then $T$ is pseudo- $A$-proper. In particular if $D$ is also convex and $T: \bar{D} \rightarrow Y$ weakly continuous, then $T$ is pseudo- $A$-proper for $Y$ not necessarily reflexive. Hence, an immediate consequence of Theorem 5.1B is the following

COROLlaRY 5.1D. Let $K$ and $M_{n}$ satisfy (3.1-2) and (3.1-4), respectively. Assume also that $X$ is reflexive and $K: X \rightarrow Y^{*}$ is demicontinuous with $R(K)$ dense in $Y^{*}$.

(D1) Let $D$ be a bounded open subset of $X$ with $0 \in D$. Suppose $Y$ is reflexive and $T: \bar{D} \rightarrow Y$ is fa-continuous, bounded, and weakly closed. If $f \in Y$ satisfies (5.1-1) of Theorem 5.1B, then there exists $x_{0} \in \bar{D}$ such that $T x_{0}=f$.

(D2) If $T: X \rightarrow Y$ is weakly continuous and $K$-coercive, then equation (5.1) is solvable for each $f \in Y$. Equation (5.1) is weakly approximationsolvable if it is uniquely solvable.

(a2) K-monotone mappings. Generalizing the notion of a monotone and a $J$-monotone mapping, we say that:

$$
\text { " } T: X \rightarrow Y \text { is } K \text {-monotone if }(T x-T y, K(x-y)) \geqq 0 \quad \text { for } x, y \in X \text { ". }
$$

It is not hard to show that if $X$ is reflexive, $K$ a strongly and weakly continuous mapping of $X$ into $Y^{*}$ which is $\alpha$-homogeneous for some $\alpha \geqq 1$ and whose range $R(K)$ is dense in $Y^{*}$, and $T$ a demicontinuous $K$-monotone mapping of $X$ into $Y$, then:

(i) $T(\bar{B}(0, r))$ is closed in $Y$ for each $r>0$.

(ii) $T$ is pseudo-A-proper.

In view of (ii), Theorem 5.1B implies the validity of the following result.

Corollary 5.1E. Suppose $X$ is reflexive, $K$ and $M_{n}$ satisfy (3.1-2) and (3.1-4) respectively, and $K$ satisfies the additional conditions mentioned above. Let $T$ be a demicontinuous (or a weakly continuous) K-monotone mapping of $X$ into $Y$.

(E1) If there is a ball $D=B(0, d)$ such that for a given $f$ in $Y$ the inequality (5.1-1) holds, then equation (5.1) has a solution in $\bar{D}$.

(E2) If $T$ is $K$-coercive, then equation (5.1) is solvable for each $f$ in $Y$. Equation (5.1) is weakly approximation-solvable if it is uniquely solvable. 
(a3) Mappings of type (PKM). In analogy with the notion of a pseudomonotone mapping introduced by Brezis [8] we say that:

" $T: X \rightarrow Y$ is of type (PKM) if $z_{k} \rightarrow z$ in $X$ and $\lim \sup _{k}\left(T z_{k}, K\left(z_{k}-z\right)\right) \leqq 0$ imply that $(T z, K(z-x)) \leqq \lim _{\inf _{k}}\left(T z_{k}, K\left(z_{k}-x\right)\right)$ for all $x \in X$."

We add in passing that a related class of the so-called mapping with pm-property has been studied in [119].

Using a somewhat complicated argument one shows that if $X$ is reflexive, $K$ a weakly continuous mapping of $X$ into $Y^{*}$ which is also uniformly continuous on closed bounded sets in $X$ and $\alpha$-homogeneous for some $\alpha \geqq 1$ with $R(K)$ dense in $Y^{*}$, and $T$ a bounded mapping of $X$ into $Y$ which is fa-continuous and of type (PKM), then for such $T$ the assertions (i) and (ii) hold. Hence, in view of (ii), Theorem 5.1B implies

Corollary 5.1F. Suppose $X$ is reflexive, $K$ and $M_{n}$ satisfy (3.1-2) and (3.1-4) respectively, and $K$ satisfies the conditions just mentioned. If $T$ is a bounded fa-continuous mapping of $X$ into $Y$ which is of type (PKM), then the conclusions (E1) and (E2) of Corollary 5.1E hold under the corresponding boundary or coerciveness condition on $T$.

Let us add in passing that, as was shown in [121], the class of mappings introduced by Kachurovskii [61] and called in [61] quasi-K-monotone mappings is a subclass of those mappings which are pointwise pseudo$A$-proper. We will not dwell on this subject here since it lies outside the scope of our review (see [121] for further discussion).

(a4) Mappings of type (KM). Modifying the notion of a mapping of type (M) introduced by Brezis [8] we say that

" $T: X \rightarrow Y$ is said to be of type (KM) if for any sequence $\left\{z_{k}\right\}$ such that

$z_{k} \rightarrow z$ in $X, T z_{k} \rightarrow g$ for some $g$ in $Y$, and $\lim \sup _{k}\left(T z_{k}, K z_{k}\right) \leqq$ $(g, K z)$ we have $T z=g$."

Using the same conditions and arguments as in (a2) one shows that if $T: X \rightarrow Y$ is a bounded fa-continuous map which is of type (KM), then for such $T$ the assertions (i) and (ii) hold. Consequently, Theorem 5.1B implies

Corollary 5.1G. Let $X, K$ and $M_{n}$ satisfy all the conditions of Corollary 5.1E. If $T$ is a bounded fa-continuous mapping of $X$ into $Y$ which is of type (KM), then the conclusions (E1) and (E2) of Corollary 5.1E hold under the corresponding boundary or coerciveness condition on $T$.

REMARK 5.1-2. It seems that the following observation concerning Theorem 5.1B and all of its corollaries is, perhaps, in order. The surjectivity part of Theorem 5.1B and its corollaries provide the assurance that a certain operator maps onto $Y$. As was pointed out in [140] this, however, 
is by no means true for every differential operator of interest to which the above abstract results are applicable. Although Theorem 5.1B and its corollaries give a condition (others will be given in \$5.2) for an operator $T$ to be onto, they also provide a condition for an element $f$ of $Y$ to be in the range of $T$ even if $T$ is not onto. In some ways this is an interesting feature of Theorem 5.1B and all of its corollaries. This is particularly true since in many cases (see [121]) one can establish the pseudo- $A$-properness of the above mappings without the condition that they be defined on all of $X$.

5.2. Applications to fixed point and surjectivity theorems for G-operators, $P_{1}$-compact, weakly closed, and monotone type mappings. The preceding results are applied here to various classes of mappings $T$ of $D \subseteq X$ into $X^{*}$ and to mappings $T$ of $D \subseteq X$ into $X$ by specifying $Y, Y_{n}, Q_{n}, K$ and $M_{n}$ as in Remark 3.1-3.

A. Existence theorems for $T: D \subseteq X \rightarrow X^{*}$. If $X$ is a real Banach space with a shrinking Schauder basis $\left\{\phi_{i}\right\} \subset X$, then $\Gamma_{S}=\left\{X_{n}, P_{n} ; X_{n}^{\prime}, P_{n}^{*}\right\}$ determined by $\left\{\phi_{i}\right\}$ (see $\S 1.1$ ) is projectionally complete for $\left(X, X^{*}\right)$. By Remark 3.1-3, $K=I$ and $M_{n}(x)=\sum_{i=1}^{n}\left(\Phi_{i}, x\right) \Phi_{i}$ satisfy all the conditions used above. Consequently, for $T: D \subseteq X \rightarrow X^{*}$, Theorem 5.1B reduces to the following

Theorem 5.2A. Suppose $X$ has a shrinking Schauder basis.

(A1) If $D \subset X$ is bounded and open with $0 \in D, T: \bar{D} \rightarrow X^{*}$ pseudo- $A$ proper and $f \in Y$ such that $(T x, x) \geqq(f, x)$ for $x \in \partial D$, then there is $x \in \bar{D}$ such that $T x=f$.

(A2) If $T: X \rightarrow X^{*}$ is pseudo-A-proper and coercive, then equation (5.1) is solvable for each $f \in X^{*}$.

For the case when $Y=X^{*}$ and $K=I$, the $K$-monotone map becomes monotone, the map of type (PKM) reduces to pseudo-monotone, while the map of type (KM) becomes a map of type (M) in the sense of Brezis if sequences replace filters. It follows from (ii) in (a2) that every demicontinuous monotone map is an $f a$-continuous pseudo- $A$-proper map while, by (ii) of (a3) and (a4), every bounded pseudo-monotone map and every bounded map of type (M) is a bounded pseudo- $A$-proper map which is also $f a$-continuous by the results of Brezis [8]. Consequently, as a corollary of Theorem 5.2A (or special cases of Corollaries 5.1D to 5.1G) we obtain the basic existence and in some cases weakly constructive results for the four special classes of mappings.

COROLlaRY 5.2B. Let $X$ be a reflexive Banach space with a Schauder basis and $T$ a mapping of $X$ into $X^{*}$. Then:

(B1) Suppose $T$ is demicontinuous and monotone. If there exists a $d>0$ such that $(T x, x) \geqq(f, x)$ for $x \in \partial B(0, d)$, then $T x=f$ has a solution in $\bar{B}$ for each such $f \in X^{*}$. If $T$ is coercive, then $T$ is surjective. 
(B2) If $T$ is bounded and either pseudo-monotone or of type (M), then the conclusions of (B1) hold under the corresponding boundary or coerciveness conditions on $T$.

(B3) If $T$ is weakly continuous, then the conclusions of (B1) hold under the corresponding boundary or coerciveness conditions on $T$.

Equation $T x=f$ is weakly approximation-solvable if it is uniquely solvable in any of the above cases.

REMARK 5.2-1. The surjectivity part of assertion (B1) has been obtained independently by Browder [19] and Minty [90] and that of (B2) by Brezis [8]. Assertion (B3) was obtained by Shinbrot [140] for separable Hilbert spaces (see also [57] for surjectivity part). The above authors used different methods and obtained their results for reflexive spaces not necessarily having Schauder bases.

B. Existence theorems for $T: D \subseteq X \rightarrow X$. Let $X$ be a real Banach $\Pi_{1-}$ space with $X^{*}$ strictly convex and let $\Gamma_{0}=\left\{X_{n}, P_{n} ; X_{n}, P_{n}\right\}$ be a projectionally complete scheme for $(X, X)$. By Remark 3.1-3, $M_{n}=I_{n}$ and $K=J$ satisfy the conditions (3.1-2) and (3.1-4) used in Theorem 5.1B. Hence the latter theorem implies the following

THEOREM 5.2C. Let $X$ be a Banach $\Pi_{1}$-space with $X^{*}$ strictly convex.

(C1) If $D \subset X$ is bounded and open with $0 \in D, T: \bar{D} \rightarrow X$ pseudo-A-proper and $f \in X$ such that $(T x, J x) \geqq(f, J x)$ for $x \in \partial D$, then there is $x \in \bar{D}$ such that $T x=f$.

(C2) If $T: X \rightarrow X$ is pseudo-A-proper and J-coercive, then equation (5.1) is solvable for each $f \in X$.

We note that if $D$ is also convex and $f=0$, then the assertion (C1) reduces to the fixed point theorem established by de Figueiredo [36] for $G$-operators since $\tilde{T}=I-T$ is a $G$-operator in the sense of [36] means essentially that $T$ is pseudo- $A$-proper at $f=0$ and $(\tilde{T} x, J x) \leqq(x, J x)$ for $x \in \partial D$ if and only if $(T x, J x) \geqq 0$ for $x \in \partial D$.

Since $\tilde{T}=I-T$ is $A$-proper and, in particular, pseudo- $A$-proper when $T$ is $P_{1}$-compact, an immediate consequence of the assertion (C1) is the following corollary which, as will be indicated below, includes a number of classical and recent fixed point theorems.

Corollary 5.2D. Suppose $X$ satisfies the conditions of Theorem 5.2C.

(D1) If $D \subset X$ is bounded and open with $0 \in D$ and $T: \bar{D} \rightarrow X$ is $P_{1^{-}}$ compact, then $T$ has a fixed point in $\bar{D}$ provided

$$
(T x, J x) \leqq(x, J x) \text { for } x \in \partial D .
$$

(D2) If $X$ is also reflexive and $T: \bar{D} \rightarrow X$ is a bounded, fa-continuous and a weakly closed map for which (5.2-1) holds, then $T$ has a fixed point in $D$. 
The above conclusion holds, in particular, if $D=B(0, r)$ and $T(\partial B(0, r)) \subset$ $\bar{B}(0, r)$.

Remark 5.2-2. Assertion (D1) includes the fixed point theorem for $P$-compact mappings established by the writer [107], [108] and its extensions in [127] and, in particular, the fixed point theorem of Schauder [138] and Rothe [135] for $T$ compact and of Kaniel [62] for $T$ quasicompact. Since every ball-condensing mapping is also $P_{1}$-compact, the fixed point theorem of Sadovsky [136] is also included. Assertion (D2) includes the fixed point theorem of Schauder [138] for $T$ weakly continuous and $D=B(0, r)$ and its extensions due to Altman [3] for $D=B(0, r)$ and $X=H$ (see also [140]) and to de Figueiredo [36] for $D$ convex.

If the duality mapping $J: X \rightarrow X^{*}$ is assumed to be both strongly and weakly continuous, then every $J$-monotone map of $X$ into $X$ is pseudo$A$-proper and consequently from assertion (C2) of Theorem $5.2 \mathrm{C}$ we deduce as corollaries the surjectivity Theorems 1,2 and 3 in Browder and de Figueiredo [22] for $J$-monotone and $J$-coercive mappings.

It follows from Corollary $5.1 \mathrm{G}$ for $Y=X$, that under the same conditions on $J$ as in the $J$-monotone case one obtains a surjectivity theorem for an $f a$-continuous mapping of $X$ into $X$ which is $J$-coercive and of type (JM). An analogous surjectivity result holds for a map of type (PJM) provided that $J$ is assumed to be also uniformly continuous on bounded sets in $X$. One can alsn obtain the existence of solutions for the equation $T x=f$ even when $T$ is not surjective provided that an element $f$ in $X$ satisfies the inequality $(T x, J x) \geqq(f, J x)$ for all $x \in \partial B(0, d)$ and some $d>0$.

We complete this section with the following interesting corollary of Theorem 5.2C, where no condition is placed on the duality mapping $J$.

Corollary 5.2E. Let $X$ be a reflexive $\Pi_{1}$-space with $X^{*}$ strictly convex and let $T: X \rightarrow X$ be weakly continuous.

(E1) If $f \in X$ and there exists $d>0$ such that $(T x, J x) \geqq(f, J x)$ for $x \in \partial B(0, d)$, then equation $T x=f$ has a solution in $\bar{B}(0, d)$.

(E2) If $T$ is J-coercive, then T maps $X$ onto $X$.

Application of (B3) of Corollary 5.2B to nonlinear elliptic equations. To indicate the usefulness of assertion (B3) we consider the generalized BVP in $W_{p}^{m}(Q)$ for the quasi-linear equation

$$
\sum_{|\alpha|,|\beta| \leq m} D^{\beta}\left[a_{\alpha \beta}\left(x, u, D u, \cdots, D^{m-1} u\right) D^{\alpha} u\right]=f \quad\left(f \in L_{q}\right)
$$

which has been treated in [140] for the case when $p=2$ (i.e. in the case of Hilbert spaces). As in [140] we make the basic assumption:

(A) The coefficients $a_{\alpha \beta}$ are continuous functions of all their variables 
and there exists a continuous function $g$ such that for all $\alpha$ and $\beta$

$$
\left|a_{\alpha \beta}\left(x, u, D u, \cdots, D^{m-1} u\right)\right| \leq g\left(\|u\|_{m, p}\right) \text { a.e. in } Q \text {. }
$$

Let $V$ be a closed subspace of $W_{p}^{m}$ such that $\stackrel{\circ}{W}_{p}^{m} \subseteq V$ and assume that

(B) The linear imbedding of $V$ into $W_{p}^{m-1}$ is compact.

Now, condition (A) implies that the (nonlinear) form

$$
a(u, v)=\sum_{|\alpha|,|\beta| \leq m}\left\langle a_{\alpha \beta}\left(x, u, \cdots, D^{m-1} u\right) D^{\alpha} u, D^{\beta} v\right\rangle
$$

is defined for all $u, v$ in $V$ and it follows from results on Nemytskii operators (see [14], [81]) that the mapping $T: V \rightarrow V^{*}$ given by

$$
a(u, v)=(T u, v) \quad(u, v \in V)
$$

is bounded and continuous. Following the usual terminology (see [14]) we define a weak solution of (5.2-2) corresponding to $V$ as an element $u$ in $V$ such that

$$
a(u, v)=\langle f, v\rangle \text { for all } v \text { in } V .
$$

Since to a given $f$ in $L_{q}$ there corresponds a unique $w_{f}$ in $V^{*}$ by $\langle f, v\rangle=$ $\left(w_{f}, v\right)$ for all $v$ in $V$, we see that $(5.2-5)$ is equivalent to the equation

$$
T u=w_{f} \quad\left(u \in V, w_{f} \in V^{*}\right) .
$$

Equation (5.2-5) together with the restriction that $u$ lies in $V$ has the force not only of requiring that $u$ should satisfy equation (5.2-2) (at least in a weak sense) but also of imposing boundary conditions upon $u$. The choices $V=\mathscr{W}_{p}^{m}$ and $V=W_{p}^{m}$ lead to the homogeneous Dirichlet and Neumann problems for (5.2-2) respectively. Using conditions (A) and (B) and a variant of the argument used in [140], one shows that $T: V \rightarrow V^{*}$ defined by (5.2-4) is weakly continuous. Consequently, assertion (B3) of Corollary 5.2B implies the validity of the following extension of Theorem 8 in [140].

THEOREM 5.2F. Suppose that conditions (A) and (B) are satisfied. If there exists $d>0$ such that $a(u, u) \geqq\langle f, u\rangle$ for $u$ in $V$ with $\|u\|_{m, p}=d$, then (5.2-2) has a weak solution in $\bar{B}(0, d) \subset V$.

If there is a function $c(r)$ of $R^{+}$into $R$ such that $c(r) \rightarrow \infty$ as $r \rightarrow \infty$ and

$$
a(u, u) \geqq c(\|u\|)\|u\| \quad \text { for all } u \text { in } V,
$$

then (5.2-2) has a weak solution for each $f \in L_{\alpha}$.

5.3. Equations involving uniform limits of A-proper type mappings. The following special case of Theorem 2 in [121] will illustrate the difference between the conditions and the proofs used in obtaining a surjectivity theorem for a non- $K$-coercive uniform limit $T: X \rightarrow Y$ of a certain sequence of pseudo- $A$-proper or $A$-proper mappings, respectively. 
Condition $(5.3++)$. If $\left\{x_{n}\right\} \subset X$ is any bounded sequence such that $T x_{n} \rightarrow g$ for some $g$ in $Y$, then there is an element $x \in X$ such that $T x=g$.

THEOREM 5.3A. Let $(X, Y), \Gamma, K$, and $M_{n}$ satisfy the conditions of Theorem 5.1B. Let $T: X \rightarrow Y$ be fa-continuous, satisfy condition $(5.3++)$ and suppose there exists a bounded fa-continuous map $F: X \rightarrow Y$ such that $F$ is $\alpha$-homogeneous for some $\alpha \geqq 1$ and

(A1) $T_{\mu}=T+\mu F$ is pseudo-A-proper for each $\mu>0$.

(A2) $(F x, K x) \geqq\|x\|^{\alpha}\|K x\|$ for all $x \in X$.

(A3) There exists $c>0$ such that $(T x, K x) \geqq-c\|K x\|$ for $x \in X$.

(A4) $\|T x\| \rightarrow \infty$ as $\|x\| \rightarrow \infty$.

Then $T(X)=Y$.

The proof of Theorem 5.3A consists in using (A2) and (A3) to show that to each $\mu_{k}>0\left(\mu_{k} \rightarrow 0\right)$ and $f \in Y$ there exists $r_{\mu_{k f}}>0$ such that $\left(T_{\mu_{k}}(x)\right.$, $K x) \geqq(f, K x)$ for $x \in \partial B\left(0, r_{\mu_{k f}}\right)$. Hence, in view of (A1), Theorem 5.1B implies the existence of $x_{k} \in B\left(0, r_{\mu_{k}}\right)$ such that $T\left(x_{k}\right)+\mu_{k} F\left(x_{k}\right)=f$. In virtue of (A4), $\left\{x_{k}\right\}$ is bounded and so $T\left(x_{k}\right) \rightarrow f$. Hence, by condition $(5.3++)$, there exists $x \in X$ such that $T x=f$.

Theorem 5.3A can be used (see [121]) to obtain a surjectivity theorem for $T: X \rightarrow Y$ satisfying (A4) for mappings discussed in (a1)-(a4) of $\$ 5.1$. Indeed, in (a2), (a3) and (a4) one shows in a simple way that in addition to (i), which implies the condition $(5.3++)$ of Theorem 5.3A, the map $T+\mu F: X \rightarrow Y$ is pseudo- $A$-proper for $F$ satisfying suitable conditions.

We restrict ourselves to the case when $Y=X^{*}, K=I$, and $F=J$ and to the case when $Y=X, K=J$, and $F=I$, where $J$ is a given duality mapping. Note that in some cases it is possible and more convenient to take linear mappings for $K$ and $F$.

COROLlary 5.3B. Let $X$ be a Banach space with a shrinking Schauder basis and with $X^{*}$ strictly convex. Let $T: X \rightarrow X^{*}$ be an fa-continuous map which satisfies condition $(5.3++)$ and is such that

(B1) $T_{\mu}=T+\mu \mathrm{J}$ is pseudo-A-proper for each $\mu>0$.

(B2) There exists $c>0$ such that $(T x, x) \geqq-c\|x\|$ for $x \in X$.

(B3) $\|T x\| \rightarrow \infty$ as $\|x\| \rightarrow \infty$.

Then, $T(X)=X^{*}$.

An immediate consequence of Corollary 5.3B is the following corollary (see [121]).

CoRollary 5.3C. Let $X$ be a reflexive Banach space with a Schauder basis and with $X^{*}$ strictly convex and let $T: X \rightarrow X^{*}$ satisfy condition (B3)

(C1) If $T$ is hemicontinuous and monotone, then $T(X)=X^{*}$.

(C2) If $T$ is bounded, pseudo-monotone, and (B2) holds, then $T(X)=X^{*}$. 
(C3) If $J$ is also weakly continuous and $T$ bounded, of type (M) and (B2) holds, then $T(X)=X^{*}$.

Remark 5.3-1. Assertion (C1) was essentially proved in [19]. Assertions (C2) and (C3) were given in [121]. If, as in [37], we assume the existence of $\alpha: R^{+} \rightarrow R^{+}$with $\alpha(t) \rightarrow \infty$ as $t \rightarrow \infty$ such that $\|T(x)-t T(-x)\| \geqq$ $\alpha(\|x\|)$ for $t \in[0,1]$ and $\|x\| \geqq r$ for some $r>0$, then for $t=0$ we have $\|T x\| \geqq \alpha(\|x\|)$ for all $x$ and so Theorem 2 in [37] follows from (C1) without the assumption that $T$ is bounded or even continuous.

Another corollary of Theorem 5.3A is the following for $T: X \rightarrow X$.

Corollary 5.3D. Let $X$ be a Banach $\Pi_{1}$-space with $X^{*}$ strictly convex. Let $T: X \rightarrow X$ be an fa-continuous mapping which satisfies condition $(5.3++)$ and for which:

(D1) $T_{\mu}=T+\mu I$ is pseudo-A-proper for each $\mu>0$.

(D2) There exists $c \geqq 0$ such that $(T x, J x) \geqq-c\|J x\|$ for $x \in X$.

(D3) $\|T x\| \rightarrow \infty$ as $\|x\| \rightarrow \infty$.

Then $T(X)=X$.

To illustrate the use of Corollary 5.3D we state the following new result for $J$-monotone mappings.

Corollary 5.3E. Let $X$ satisfy the conditions of Corollary 5.3D and let $J$ be both strongly and weakly continuous. If $T: X \rightarrow X$ is J-monotone, demicontinuous and (D3) holds, then $T(X)=X$.

Corollary 5.3E holds, in particular, when $X=l_{p}$ since $l_{p}$ 's have duality maps $v$ hich are strongly and weakly continuous.

If $\mathrm{n}$ Theorem $5.3 \mathrm{~A}$ it is assumed that $T_{\mu}=T+\mu F$ is $A$-proper for each $\mu>0$, then the recent surjectivity theorem of Fitzpatrick (see [41, Theorem 1.1]) admits the following generalization to mappings $T: X \rightarrow Y$ with the corresponding change in conditions (A3) and (A4) and for Banach spaces $(X, Y)$ having a projectionally complete scheme $\Gamma$.

Theorem 5.3F. Let $(X, Y), \Gamma, K$ and $M_{n}$ satisfy the conditions of Theorem 5.1B. Let $T: X \rightarrow Y$ be fa-continuous and satisfy condition $(5.3++)$ and suppose there exists a bounded fa-continuous mapping $F: X \rightarrow Y$ such that

(F1) $T_{\mu}=T+\mu F$ is A-proper for each $\mu>0$.

(F2) $(F x, K x) \geqq 0$ for all $x$ in $X$.

(F3) There exists $r_{0}>0$ such that $(A x, K x) \geqq 0$ for $\|x\| \geqq r_{0}$.

(F4) $T$ satisfies condition (3.1+) (i.e., if $\left\{x_{k}\right\} \subset X$ is such that $T\left(x_{k}\right) \rightarrow g$ for some $g \in Y$ then $\left\{x_{k}\right\}$ is bounded).

Then $T(X)=Y$. 
To indicate the proof (see [125] for details), note that to a given $f \in Y$ there exist $r>r_{0}$ and $c>0$ such that $\|T x-t f\| \geqq c$ for $t \in[0,1]$ and $\|x\|=r$. Since $F$ is bounded, we can choose $\mu_{0}>0$ such that $\|(T+\mu F) x-t f\| \geqq c / 2$ for all $t \in[0,1], \mu \in\left(0, \mu_{0}\right)$ and $\|x\|=r$. For any fixed $\mu \in\left(0, \mu_{0}\right)$, (F1) implies the existence of $n_{0} \geqq 1$ and $\gamma>0$ such that $\left\|Q_{n} T_{\mu} x-t Q_{n} f\right\| \geqq \gamma$ for all $x \in X_{n}$ with $\|x\|=r, n \geqq n_{0}$, and $t \in[0,1]$. The homotopy theorem applied to $H_{t}^{n}(x)=t Q_{n} T_{\mu}(x)+(1-t) M_{n}(x)$ for $x \in \bar{B}_{n}(0, r)$ and $t \in[0,1]$ for $n \geqq n_{0}$ shows that $\operatorname{deg}\left(H_{t}^{n}, B_{n}(0, r), 0\right) \neq 0$ for all $t \in[0,1]$. It follows from this and the $A$-properness of $T_{\mu}$ that to each $\mu_{k} \in\left(0, \mu_{0}\right)$ with $\mu_{k} \rightarrow 0$ there exist $x_{k} \in B(0, r)$ such that $T\left(x_{k}\right)+\mu_{k} F\left(x_{k}\right)=f$. Hence, $T\left(x_{k}\right) \rightarrow f$ as $k \rightarrow \infty$ and so there exists $x \in X$ such that $T x=f$.

It should be added that Theorems $5.3 \mathrm{~A}$ and $5.3 \mathrm{~F}$ are different although a number of surjectivity theorems for various special classes of mappings can be deduced from either one of them.

In view of Theorem 5.3F, the fixed point theory for $P$-compact mappings, the generalized degree theory for uniform limits of $A$-proper mappings (see [16], [38]), and for other reasons (see [124]), it is important to find classes of mappings $T: D \subseteq X \rightarrow Y$ such that for a suitable choice of $F: X \rightarrow Y$ the mapping $T_{\mu}=T+\mu F$ is $A$-proper with respect to a given scheme $\Gamma$. We mention here in historical order some of the classes for which the above has been shown to be true.

In [108], [110] the author showed that if $H$ is a Hilbertspace and $T: H \rightarrow H$ is monotone and either continuous or bounded and demicontinuous, then $T_{\mu}=T+\mu I$ is $A$-proper for each $\mu>0$. When $X$ is a Banach $\Pi_{1}$-space and $S: X \rightarrow X$ is $\alpha$-contractive with $\alpha<1$ and $T=I-S$, then $T_{\mu}=T+\mu I$ is $A$-proper for each $\mu>0$. It follows from Theorem 2.3 in [115] that if $X$ and $X^{*}$ are reflexive with property $(\mathrm{H})$ and $T: X \rightarrow X^{*}$ is monotone and either continuous or bounded and demicontinuous, then $T_{\mu}=T+\mu J$ is $A$-proper for $\mu>0$, where $J: X \rightarrow X^{*}$ is any duality mapping. Moreover, if $J$ is also weakly continuous and $T: X \rightarrow X$ is $J$-monotone, then $T_{\mu}=T+\mu I$ is $A$-proper for $\mu>0$. Each of the above assertions remains true for the mapping $T+C+\mu I$ or $T+C+\mu J$ when $C$ is compact.

In [19] Browder showed that if $X$ is a separable reflexive Banach space with $X$ and $X^{*}$ locally uniformly convex, $J$ a normalized duality map, and $T: X \rightarrow X^{*}$ pseudo-monotone, then $T_{\mu}=T+\mu J$ satisfies condition $\left(\mathrm{S}_{+}\right)$for each $\mu>0$ and hence $A$-proper if $T$ is also bounded and demicontinuous.

In [12], [15] Browder initiated the study of intertwining mappings of semicontractive and semi-J-monotone type (see [34]) which became a subject of study by many authors (see [19], [42], [122] for references). Here we state the definition and mention only those results which are relevant to our problem. 
Definition 5.3G. Let $X$ be a Banach space and $T$ a mapping of $X$ into $X$.

(G1) $T$ is semi-J-monotone if there exists a $V: X \times X \rightarrow X$ such that $T(x)=V(x, x)$ for $x \in X, V(\cdot, x)$ is $J$-monotone and demicontinuous and $V(x, \cdot)$ is completely continuous for each fixed $x \in X$.

(G2) $T$ is strictly (weakly) semicontractive if $V: X \times X \rightarrow X$ is continuous and if, for each fixed $x \in X, V(\cdot, x)$ is $l$-contractive with constant $l<1$ $(l=1)$ and $V(x, \cdot)$ is compact.

It was shown by Edmunds and Webb [34] (see also [154]) that if $X$ is a reflexive $\Pi_{1}$-space, $X^{*}$ strictly convex, $J: X \rightarrow X^{*}$ strongly and weakly continuous and $T: X \rightarrow X$ semi- $J$-monotone, then $T_{\mu}=T+\mu I$ is $A$-proper for each $\mu>0$. In [155] Webb showed that if $T$ is strictly semicontractive, then $T$ is ball-condensing while the author has shown in [122] that if $T$ is strictly or weakly semicontractive, then $T$ is $l$-ball-contractive with $l \leqq 1$ depending on whether $T$ is strictly or weakly semicontractive.

Now, if $X$ is also a $\Pi_{1}$-space, $L: X \rightarrow X$ is 1-ball-contractive and $T=I-L$, then $T_{\mu}=T+\mu I$ is $A$-proper for each $\mu>0$.

Thus Theorem $5.3 \mathrm{~F}$ is applicable to the above classes of mappings. As an example, we state the following corollary which can be used to deduce a number of results from [127], [157] obtained there by different and more complicated methods but for more general spaces.

Corollary 5.3H. Let $X$ be a Banach $\Pi_{1}$-space and let $L: X \rightarrow X$ be a ball-condensing mapping such that $(L x, J x) \leqq(x, J x)$ for $\|x\| \geqq r_{0}$ and some $r_{0} \geqq 0$. If $T=I-L$ satisfies conditions $(3.1+)$ and $(5.3++)$, then $T(X)=X$.

Extending the result of Browder [19] to $T: X \rightarrow Y$ the author introduced in [119] the extension of the notion of pseudo-monotonicity in such a way as to be more in consonance with the concept of an $A$-proper mapping.

Definition 5.3I. A continuous $T: X \rightarrow Y$ is said to have the pmproperty if for any sequence $\left\{x_{n_{j}} \mid x_{n_{j}} \in X_{n_{j}}\right\}$ for which $x_{n_{j}} \rightarrow x$ and $\lim \sup _{j}\left(T x_{n_{j}}-T P_{n_{j}} x, K\left(x_{n_{j}}-P_{n_{j}} x\right)\right) \leqq 0$ we have the relation

$$
(T x, K(x-v)) \leq \lim _{j} \inf \left(T x_{n_{j}}, K\left(x_{n_{j}}-P_{n_{j}} v\right)\right) \text { for each } v \in X .
$$

As a special case of Proposition 4.4 in [119] we can state that if $X$ is reflexive, if $K: X \rightarrow Y^{*}$ is weakly continuous at 0 with $Q_{n}^{*} K x=K x$ for $x \in X_{n}$, and if $T: X \rightarrow Y$ has the $p m$-property, then $T_{\mu}=T+\mu F$ is $A$-proper whenever $F$ is a continuous $K$-monotone mapping of $X$ into $Y$ which satisfies the modified condition (S). The $A$-properness of $T+\mu F: X \rightarrow Y$ under different conditions on $T$ and $F$ was also obtained in [119]. Consequently, Theorem $5.3 \mathrm{~F}$ is valid for these classes of mappings $T$ provided, of course, that $T$ also satisfies conditions (F3) and (F4). 
We complete this section by deducing from Theorem $5.3 \mathrm{~F}$ the surjectivity theorem in [41] mentioned above.

Definition 5.3J. (J1) $T: X \rightarrow X^{*}$ is called semimonotone if there exists $V: X \times X \rightarrow X^{*}$ such that $T(x)=V(x, x)$ for $x \in X, V(x, \cdot)$ is monotone and hemicontinuous and $V(\cdot, x)$ is completely continuous for each fixed $x \in X$.

(J2) $T: X \rightarrow X^{*}$ satisfies condition (P) if $\lim \sup _{j}\left(T x_{j}, x_{j}-x\right) \geqq 0$ whenever $x_{j} \rightarrow x$ in $X$.

The study of semimonotone mappings was initiated by Browder [11] and that of operators satisfying condition $(\mathrm{P})$ by Hess [54]. It was shown by Fitzpatrick [41] that bounded pseudo-monotone mappings and semimonotone mappings are demicontinuous and satisfy condition $(\mathrm{P})$. On the other hand, it was observed by Hess [54] that if $X$ is reflexive, $X$ and $X^{*}$ locally uniformly convex and $T: X \rightarrow X^{*}$ satisfies condition $(\mathrm{P})$ then $T_{\mu}=T+\mu J$ satisfies condition $\left(\mathrm{S}_{+}\right)$and thus is $A$-proper for each $\mu>0$ by the result of Browder [19]. Consequently, Theorem 5.3F implies the validity of the following corollary for $\left(X, X^{*}\right)$ having a projectionally complete scheme $\Gamma_{1}$.

Corollary 5.3K. Let $X$ be a reflexive Banach space and suppose $T: X \rightarrow X^{*}$ is demicontinuous and satisfies condition (P). Assume that $T$ satisfies condition $(5.3++)$ and that $(T x, x) \geqq 0$ for all $\|x\| \geqq r_{0}$ and some $r_{0}>0$. If $T$ satisfies condition (3.1+), then $T(X)=X^{*}$.

In its present form Corollary 5.3K was proved by Fitzpatrick [41] while some of its special cases had been established earlier by Browder [14], Brezis [8], and Rockafellar [134]. The above authors used different arguments and established their results for spaces which need not have projectionally complete schemes. We add that as has been noted in [125], Theorem $5.3 \mathrm{~F}$ and its corollaries, including Corollary $5.3 \mathrm{~K}$, are in fact valid for separable spaces when the $A$-properness of a mapping is defined in terms of more general approximation schemes.

5.4. Surjectivity theorems for odd limits of A-proper mappings. In this section we indicate how the surjectivity theorems of Pokhodjayev [130] and the author [115] for odd $A$-proper mappings have been extended to odd mappings which are uniform limits of $A$-proper mappings.

The first result in this direction was obtained by Browder [19] for an odd, bounded, continuous pseudo-monotone mapping $T: X \rightarrow X^{*}$ by using the degree theory for $A$-proper mappings developed in Browder and Petryshyn [24], [25]. The results of [130], [115], [19] were then further extended in various directions in [16], [38], [44], [54], [92], [119], [120], [127]. The recent result of Fitzpatrick [41] for $T: X \rightarrow X^{*}$ satisfying conditions $(3.1+)$ and $(P)$ provides a further generalization of earlier results. 
To discuss the recent surjectivity theorems for odd $T: X \rightarrow Y$ which are uniform limits of $A$-proper maps we use the writer's approach from [115], [120] which is based on the direct use of $A$-proper mapping theory and not on the theory of generalized topological degree for maps in Banach spaces as used in [16], [19], [38], [119]. In fact, the approach used recently in [41] is similar to the technique used in [120]. As will be seen from its special cases, the following surjectivity theorem obtained by the writer in [120] (using Theorem 3.2D) appears to be the most general result for odd $T: X \rightarrow Y$ which are uniform limits of $A$-proper maps, at least in the case when the Banach spaces $(X, Y)$ have projectionally complete schemes $\Gamma$ (or even when they are separable, [125]). It includes not only the earlier results from [16], [19], [38], [119] as was noted in [120] but, as will be seen below, it also includes the recent results from [121] and [41].

Theorem 5.4A. Let $T: X \rightarrow Y$ be fa-continuous, odd on $X-B\left(0, x_{0}\right)$ and let it satisfy condition $(5.3++)$. Suppose there is a sequence of $A$-proper maps $T_{j}: X \rightarrow Y$ such that:

(A1) $T_{j}$ converges uniformly to $T$ on each ball $B(0, r)$.

(A2) $T_{j}$ is odd on $X-B\left(0, r_{0}\right)$ for each $j$.

(A3) To each $f \in Y$ there exist $r_{f} \geqq r_{0}$ and $N_{f} \geqq 1$ such that $T_{j}(x)-t f \neq 0$ for $x \in \partial B\left(0, r_{f}\right), t \in[0,1]$ and $j \geqq N_{f}$.

Then $T(X)=Y$.

It turns out that when $T: X \rightarrow Y$ satisfies condition $(3.1+)$, then Theorem $5.4 \mathrm{~A}$ implies the validity of the following new result, which forms the basis for applications to special classes of mappings.

Theorem 5.4B. Let $T: X \rightarrow Y$ be fa-continuous, odd on $X-B\left(0, r_{0}\right)$ and satisfy condition $(5.3++)$. Suppose there exists a bounded fa-continuous mapping $F: X \rightarrow Y$ such that $F$ is odd on $X-B\left(0, r_{0}\right)$ and $T_{\mu}=T+\mu F$ is $A$-proper for each $\mu>0$. Then if $T$ satisfies condition $(3.1+), T(X)=Y$.

Proor. To deduce Theorem 5.4B from Theorem 5.4A note that, since $T$ satisfies condition $(3.1+)$, to each $f \in Y$ there exist $r>r_{0}$ and $c>0$ such that (3.1-7) holds and, therefore, since $F$ is bounded one finds $\mu_{0}>0$ such that $\left\|T_{\mu}(x)-t f\right\| \geqq c / 2$ for $x \in \partial B(0, r), t \in[0,1]$ and $\mu \in\left(0, \mu_{0}\right)$. Thus, if $F_{j} \equiv T+\mu_{j} F$ with $\mu_{j}>0$ and $\mu_{j} \rightarrow 0$, then $\left\{F_{j}\right\}$ thus chosen satisfies (A1) to (A3) with $r_{f}=r$ and $N_{f}$ such that $\left\{\mu_{j}\right\} \subset\left(0, \mu_{0}\right)$ for $j \geqq N_{f}$. Hence, $T(X)=Y$ by Theorem 5.4A. Q.E.D.

It is obvious that Theorem 5.4B is applicable to various classes of mappings $T: X \rightarrow Y$ discussed at the end of $\$ 5.3$ which have the property that $T_{\mu}=T+\mu F$ is $A$-proper for each $\mu>0$ for a suitable choice of $F: X \rightarrow Y$ depending on $Y, T$ and/or $K: X \rightarrow Y^{*}$. Of course, it must be assumed that $T$ and $F$ are odd on $X-B\left(0, r_{0}\right)$ and that $T$ satisfies condition (3.1+) 
or some stronger but more easily verifiable conditions. It is easy to check that condition $(3.1+)$ is implied by any one of the following conditions:

(\#) $\|T x\| \rightarrow \infty$ as $\|x\| \rightarrow \infty$ (or $T^{-1}(Q)$ is bounded whenever $Q \subset Y$ is bounded).

(\#\#) $0 \notin \operatorname{cl} T\left(\partial B\left(0, r_{0}\right)\right)$ and $T(t x)=t^{\alpha} T x$ for $\|x\| \geqq r_{0}, t>1$ and some $\alpha>0$.

In view of this, the corresponding surjectivity theorems of Browder [16], [19] for pseudo-monotone maps $T: X \rightarrow X^{*}$, Theorem 9 of the author [119] for $T: X \rightarrow Y$ having the $p m$-property and Proposition 2.7 of Fitzpatrick [38] follow from Theorem 5.4B. Moreover, the writer's Theorem 4 in [120] and all of its corollaries follow also from Theorem 5.4B.

We complete this section by stating two corollaries of Theorem 5.4B for mappings $T: X \rightarrow X^{*}$ and $T: X \rightarrow X$ which have recently been extensively studied.

COROLlary 5.4C. Let $\left(X, X^{*}\right)$ be a reflexive couple with a projectionally complete scheme $\Gamma$. Let $T: X \rightarrow X^{*}$ be a demicontinuous mapping which satisfies condition $(\mathrm{P})$ and condition $(5.3++)$ and which is odd on $X-$ $B\left(0, r_{0}\right)$. Then, if $T$ satisfies condition $(3.1+), T(X)=X^{*}$.

Some special cases of Corollary $5.4 \mathrm{C}$ were obtained earlier as noted above, while in its present form it was proved in [41] without the assumption that $\left(X, X^{*}\right)$ have a projectionally complete scheme. We add in passing that the arguments used to prove Theorem 5.4B and thus Corollary $5.4 \mathrm{C}$ are also valid for separable spaces provided the $A$-properness of $T$ is defined in terms of more general schemes.

Corollary 5.4D. Let $X$ be a Banach $\Pi_{1}$-space with $X^{*}$ strictly convex and let $T: X \rightarrow X$ be fa-continuous, odd on $X-B\left(0, r_{0}\right)$ and satisfy condition $(5.3++)$. Suppose that $T=T+\mu I$ is A-proper for each $\mu>0$. Then, if $T$ satisfies condition $(3.1+), T(X)=X$.

REMARK 5.4-1. The conditions of Corollary 5.4D are certainly satisfied if $T$ is $P_{1}$-compact and, in particular, ball-condensing and satisfies condition $(3.1+)$. The corollary also holds if $T$ is 1-ball-contractive and satisfies conditions $(5.3++)$ and $(3.1+)$. Hence Corollary 5.4D includes (for the narrower class of Banach spaces considered here) the corresponding part of Theorem 1.1 in [127] and the various corollaries stated there. If $X$ is also reflexive, $X^{*}$ has property $(\mathrm{H})$ and a given duality mapping $J: X \rightarrow X^{*}$ is weakly continuous, then Corollaries $1 \mathrm{~b}, 2 \mathrm{~b}$, and $3 \mathrm{~b}$ in [120] follow from Corollary 5.4D. The semi-J-monotone mappings studied in [34] can also be studied by means of Corollary 5.4D.

REMARK 5.4-2. In his Rutgers Ph.D. thesis under preparation P. Miloyevich extended the fixed point theorems of $\S 1.2$ and some surjectivity 
theorems of Chapters 3 and 4 to multivalued $P$-compact and $A$-proper maps.

\section{REFERENCES}

1. S. Agmon, Lectures on elliptic boundary value problems, Van Nostrand Math. Studies, no. 2, Van Nostrand, Princeton, N.J., 1965. MR 31 \#2504.

2. M. S. Al'tman, A fixed point theorem in Banach space, Bull. Acad. Polon. Sci. Cl. III 5 (1957), 89-92. MR 19, 297.

3. - A fixed point theorem in Hilbert space, Bull. Acad. Polon. Sci. Cl. III 5 (1957), 19-22. MR 19, 297.

4. H. Amann, Fixed points of asymptotically linear maps in ordered Banach spaces, J. Functional Analysis 14 (1973), 162-171. 1972.

6. L. P. Belluce and W. A. Kirk, Fixed-point theorems for certain classes of nonexpansive mappings, Proc. Amer. Math. Soc. 20 (1969), 141-146. MR 38 \#1663.

7. Ju. G. Borisovic and Ju. I. Sapronov, A contribution to the topological theory of condensing operators, Dokl. Akad. Nauk SSSR 183 (1968), 18-20=Soviet Math. Dokl. 9 (1968), 1304-1307. MR 38 \#6414.

8. H. R. Brezis, Équations et inéquations non-linéaires dans les espaces vectoriels en dualité, Ann. Inst. Fourier (Grenoble) 18 (1968), fasc. 1, 115-175. MR 42 \#5113.

9. H. R. Brezis and M. Sibony, Méthodes d'approximation et d'itération pour les opérateurs monotones, Arch. Rational Mech. Anal. 28 (1967/68), 59-82. MR 36 \#3177.

10. F. E. Browder, The solvability of non-linear functional equations, Duke Math. J. 30 (1963), 557-566. MR 27 \#6133.

11. - Mapping theorems for noncompact nonlinear operators in Banach spaces, Proc. Nat. Acad. Sci. U.S.A. 54 (1965), 337-342. MR 31 \#5113.

12. - Fixed point theorems for nonlinear semicontractive mappings in Banach spaces, Arch. Rational Mech. Anal. 21 (1965/66), 259-269. MR 34 \#641.

13. - Approximation-solvability of nonlinear functional equations in normed linear spaces, Arch. Rational Mech. Anal. 26 (1967), 33-42. MR 36 \#3185.

14. - Existence theorems for nonlinear partial differential equations, Proc. Sympos. Pure Math., vol. 16, Amer. Math. Soc., Providence, R.I., 1970, pp. 1-60. MR 42 \#4855.

15. - Semicontractive and semiaccretive nonlinear mappings in Banach spaces, Bull. Amer. Math. Soc. 74 (1968), 660-665. MR 37 \#5742.

16. _- Nonlinear elliptic boundary value problems and the generalized topological degree, Bull. Amer. Math. Soc. 76 (1970), 999-1005. MR 41 \#8818.

17. - Nonlinear mappings of analytic type in Banach spaces, Math. Ann. 185 (1970), 259-278. MR 41 \#4318.

18. - Normal solvability and $\phi$-accretive mappings of Banach spaces, Bull. Amer. Math. Soc. 78 (1972), 186-192. MR 46 \#6113.

19. - Nonlinear operators and nonlinear equations of evolution in Banach spaces, Proc. Sympos. Pure Math., vol. 18, part II, Amer. Math. Soc., Providence, R.I., (to appear).

20. - Topology and nonlinear functional equations, Studia Math. 31 (1969), 189-204.

21. - Remarks on nonlinear functional equations. III, Illinois J. Math. 9 (1965), 617-622. MR 32 \#2941. 
22. F. E. Browder and D. G. de Figueiredo, J-monotone nonlinear operators in Banach spaces, Nederl. Akad. Wetensch. Proc. Ser. A 69=Indag. Math. 28 (1966), 412-420. MR 34 \#4957.

23. F. E. Browder and R. D. Nussbaum, The topological degree for noncompact nonlinear mappings in Banach spaces, Bull. Amer. Math. Soc. 74 (1968), 671-676. MR 38 \#583.

24. F. E. Browder and W. V. Petryshyn, Approximation methods and the generalized topological degree for nonlinear mappings in Banach spaces, J. Functional Analysis 3 (1969), 217-245. MR 39 \#6126.

25. - The topological degree and Galerkin approximations for noncompact operators in Banach spaces, Bull. Amer. Math. Soc. 74 (1968), 641-646. MR 37 \#4678.

26. L. Cesari, Functional analysis and Galerkin's method, Michigan Math. J. 11 (1964), 385-414. MR 30 \#4047.

27. J. Cronin-Scanlon, Analytic functional mappings, Ann. of Math. (2) 58 (1953), 175-181. MR 15, 234.

28. —_ Fixed points and topological degree in nonlinear analysis, Math. Surveys, no. 11, Amer. Math. Soc., Providence, R.I., 1964. MR 29 \#1400.

29. - A definition of degree for certain mappings in Hilbert space, Amer. J. Math. 73 (1951), 763-772. MR 13, 662.

30. K. Deimling, Fixed points of generalized P-compact operators, Math. Z. 115 (1970), 188-196. MR 41 \#9073.

31. L. Dozo, Opérateurs non-expansifs, P-compacts et propriétés géométriques de la norme, Doctoral Thesis, Université Libre de Bruxelles, 1969-1970.

32. A. V. Džiškariani, The least square and Bubnov-Galerkin methods, ž. Vyčisl. Mat. i Mat. Fiz. 8 (1968), 1110-1116. (Russian) MR 38 \#6418.

33. - On the stability of approximation methods of the variational type, $\check{\mathrm{Z}}$. Vycisl. Mat. i Mat. Fiz. 11 (1971), 569-579. (Russian).

34. D. E. Edmunds and J. R. L. Webb, A Leray-Schauder theorem for a class of nonlinear operators, Math. Ann. 182 (1969), 207-212. MR 40 \#7895.

35. D. G. de Figueiredo, Topics in nonlinear functional analysis, Lecture Series, no. 48, University of Maryland, College Park, Md., 1967.

36. —_ Fixed-point theorems for nonlinear operators and Galerkin approximations, J. Differential Equations 3 (1967), 271-281. MR 34 \#6578.

37. D. G. de Figueiredo and C. P. Gupta, Solvability of nonlinear equations of Hammerstein type (to appear).

38. P. M. Fitzpatrick, A generalized degree for uniform limits of A-proper mappings, J. Math. Anal. Appl. 35 (1971), 536-552. MR 43 \#6788.

39. —_, A-proper mappings and their uniform limits, Ph.D. Thesis, Rutgers University, New Brunswick, N.J., 1971.

40. - A-proper mappings and their uniform limits, Bull. Amer. Math. Soc. 78 (1972), 806-809. MR 46 \#2512.

41. - Surjectivity results for nonlinear mappings from a Banach space to its dual, Math. Ann. 204 (1973), 177-188.

42. - On the structure of the set of solutions of equations involving A-proper mappings, Trans. Amer. Math. Soc. 189 (1974), 107-131.

43. K. O. Friedrichs, Spektraltheorie halbeschränkter Operatoren, Math. Ann. 109 (1934), 465-487, 685-713.

44. S. Fuxík, Fredholm alternative for nonlinear operators in Banach spaces and its applications to differential and integral equations, Casopis Pěst. Mat. 96 (1971), 371-390.

45. S. Fučik and J. Nečas, On the existence of Schauder bases in Sobolev spaces, Comment. Math. Univ. Carolinae 13 (1972), 163-175. 
46. G. M. Gončarov, On some existence theorems for the solutions of a class of nonlinear operator equations, Mat. Zametki 7 (1970), 229-237=Math. Notes 7 (1970), 137-141. MR 41 \#7499.

47. S. Granas, On a class of nonlinear mappings in Banach spaces, Bull. Acad. Polon. Sci. Cl. III 5 (1957), 867-871. (Russian) MR 19, 968.

48. - Introduction to topology of functional spaces, University of Chicago Lecture Notes, 1961.

49. R. D. Grigorieff, Zur Theorie linearer approximations regularer Operatoren. I, II, Math. Nachr. (Erscheint).

50. - - Vereallgemeinerte approximativ kompakte Operatoren, Habilitationsschrift, Frankfurt, 1969.

51. — U Uber die Fredholm-Alternative bei linearen approximations regularen Operatoren, Appl. Anal. 2 (1972), 217-227.

52. J. D. Hamilton, Noncompact mappings and cones in Banach spaces, Arch. Rational Mech. Anal. 48 (1972), 153-162.

53. P. Hess, On the Fredholm alternative for nonlinear functional equations in Banach spaces, Proc. Amer. Math. Soc. 33 (1972), 55-61.

54. - On nonlinear mappings of monotone type homotopic to odd operators,

J. Functional Analysis 11 (1972), 138-167.

55. S. Hildebrandt and E. Weinholtz, Constructive proofs of representation theorems in separable Hilbert space, Comm. Pure Appl. Math. 17 (1964), 369-373. MR 29 \#3881.

56. H. Jeggle, Über die Approximation von linearen Gleichungen zweiter Art und Eigenwertproblemen in Banachschen Räumen, Math. Z. (Erscheint).

57. R. I. Kačurovskiî, Nonlinear monotone operators in Banach spaces, Uspehi Mat. Nauk 23 (1968), no. 2 (140), 121-168=Russian Math. Surveys 23 (1968), no. 2, 117-165. MR 37 \#2045.

58. - On the Fredholm theory for nonlinear operator equations, Dokl. Akad. Nauk SSSR 192 (1970), 969-972=Soviet Math. Dokl. 11 (1970), 751-754. MR 42 \#911.

59. - On nonlinear operators whose ranges are subspaces, Dokl. Akad. Nauk SSSR 196 (1971), 508-511 =Soviet Math. Dokl. 12 (1971), 168-172.

60. - Approximate methods for the solution of nonlinear operator equations, Izv. Vyš̌. Užebn. Zaved. Matematika 1967, no. 12 (67), 27-37. (Russian) MR 37 \#2044.

61. - Tihonov's fixed-point principle and equations with operators weakly closed on a kernel, Dokl. Akad. Nauk SSSR 183 (1968), 517-520=Soviet Math. Dokl. 9 (1968), 1411-1414. MR 38 \#5082.

62. S. Kaniel, Quasi-compact non-linear operators in Banach space and applications, Arch. Rational Mech. Anal. 20 (1965), 259-278. MR 32 \#4575.

63. L. V. Kantorovič, Functional analysis and applied mathematics, Uspehi Mat. Nauk 3 (1948), no. 6 (28), 89-185; English transl., Nat. Bur. Standards Rep., no. 1509, U.S. Dept. of Commerce, Nat. Bur. Standards, Washington, D.C., 1952. MR 10, 380; 14, 766.

64. T. Kato, Perturbation theory for linear operators, Die Grundlehren der math. Wissenschaften, Band 132, Springer-Verlag, New York, 1966. MR 34 \#3324.

65. W. A. Kirk, Mappings of generalized contractive type, J. Math. Anal. Appl. 32 (1970), 567-572. MR 42 \#6675.

66. - On nonlinear mappings of strongly semicontractive type, J. Math. Anal. Appl. 27 (1969), 409-412. MR 39 \#6128. 
67. M. A. Krasnosel'skil, Topological methods in the theory of nonlinear integral equations, GITTL, Moscow, 1956; English transl., Macmillan, New York, 1964. MR 20 \#3464; 28 \#2414.

68. - Two remarks on the method of successive approximations, Uspehi Mat. Nauk 10 (1955), no. 1 (63), 123-127. (Russian) MR 16, 833.

69. - Convergence of Galerkin's method for nonlinear equations, Dokl. Akad. Nauk SSSR 73 (1950), 1121-1124. (Russian) MR 12, 187.

70. - Positive solutions to operator equations, Fizmatgiz, Moscow, 1962; English transl., Noordhoff, Groningen, 1964. MR 26 \#2862; 31 \#6107.

71. M. A. Krasnosel'skii and P. E. Sobolevskiir, Structure of the set of solutions of an equation of parabolic type, Ukrain. Mat. Ž. 16 (1964), 319-333; English transl., Amer. Math. Soc. Transl. (2) 51 (1966), 113-131. MR 29 \#3763.

72. M. A. Krasnosel'skiǐ, G. M. Vaĭnikko, P. P. Zabrě̌ko, Ja. B. Rutickiǐ and V. Ja. Stecenko, Approximate solution of operator equations, "Nauka", Moscow, 1969; English transl., Wolters-Noordhoff, Groningen, 1972. MR 41 \#4271.

73. M. Kravcuk, Application of the method of moments to the solution of linear differential and integral equations, Ukrain. Akad. Nauk. Kiev, 1936.

74. O. L. Ladyženskaja, On integral estimates, convergence, approximate methods, and solution in functionals for elliptic equations, Vestnik Leningrad. Univ. 13 (1958), no. 7, 60-69. (Russian) MR 20 \#5353.

75. A. D. Ljaško, The convergence of Galerkin type methods, Dokl. Akad. Nauk SSSR 120 (1958), 242-244. (Russian) MR 20 \#4346.

76. P. D. Lax and A. N. Milgram, Parabolic equations, Contributions to the Theory of Partial Differential Equations, Ann. of Math. Studies, no. 33, Princeton Univ. Press, Princeton, N.J., 1954, pp. 167-190. MR 16, 709.

77. M. Lees and M. H. Schultz, A Leray-Schauder principle for A-compact mappings and the numerical solution of non-linear two-point boundary value problems, Numerical Solutions of Nonlinear Differential Equations (Proc. Adv. Sympos., Madison, Wis., 1966), Wiley, New York, 1966, pp. 167-179. MR 35 \#819.

78. J. Leray, Topologie des espaces abstraits de M. Banach, C.R. Acad. Sci. Paris 200 (1935), 1082-1084.

79. J. Leray and J.-P. Lions, Quelques résultats de Višik sur les problèmes elliptiques non linéaires par les méthodes de Minty-Browder, Bull. Soc. Math. France 93 (1965), 97107. MR 33 \#2939.

80. J. Leray and J. Schauder, Topologie et équations fonctionnelles, Ann. Sci. Ecole Norm. Sup. 51 (1934), 45-78.

81. J.-L. Lions, Quelques méthodes de résolution des problèmes aux limites non linéaires, Dunod; Gauthier-Villars, Paris, 1969. MR 41 \#4326.

82. A. Ju. Lučka, The rate of convergence of certain projection methods for linear operator equations, Ukrain. Mat. Z. 23 (1971), 307-317. (Russian) MR 44 \#2054.

83. J. T. Marti, Introduction to the theory of bases, Springer Tracts in Natural Philosophy, vol. 18, Springer-Verlag, Berlin and New York, 1970.

84. A. E. Martynjuk, Variational methods in boundary value problems for weakly elliptic equations, Dokl. Akad. Nauk SSSR 126 (1959), 1222-1225. (Russian) MR 22 \#4867.

85. V. A. Medvedev, On the convergence of the Bubnov-Galerkin method, Prikl. Mat. Meh. 27 (1963), 1148-1151=J. Appl. Math. Mech. 27 (1963), 1769-1774. MR 29 \#1753.

86. S. G. Mihlin, Variational methods in mathematical physics, GITTL, Moscow, 1957; German transl., Akademie-Verlag, Berlin, 1962; English transl., Macmillan, New York, 1964. MR 22 \#1981; 25 \#4658; 30 \#2712. 
87. - On the convergence of Galerkin's method, Dokl. Akad. Nauk SSSR 61 (1948), 197-199. (Russian) MR 10, 129.

88. —- The numerical performance of variational methods, "Nauka", Moscow, 1966; Wolters-Noordhoff, Groningen, 1971. MR 34 \#3747; 43 \#4236.

89. G. J. Minty, Monotone (nonlinear) operators in Hilbert space, Duke Math. J. 29 (1962), 341-346. MR 29 \#6319.

90. - On a "monotonicity" method for the solution of nonlinear equations in Banach spaces, Proc. Nat. Acad. Sci. U.S.A. 50 (1963), 1038-1041. MR 28 \#5358.

91. J. Nečs, Remark on the Fredholm alternative for nonlinear operators with application to nonlinear integral equations of generalized Hammerstein type, Comment. Math. Univ. Carolinae 13 (1972), 109-120. MR 46 \#4301.

92. —_ Fredholm alternative for nonlinear operators and applications to partial differential equations and integral equations, Casopis Pěst. Mat. 97 (1972), 65-71, 94. MR 46 \#7994.

93. R. D. Nussbaum, The fixed point index and fixed point theorems for $k$-set-contractions, Ph.D. Dissertation, University of Chicago, Chicago, Ill., 1969.

94. - - The radius of the essential spectrum, Duke Math. J. 37 (1970), 473-478. MR 41 \#9028.

95. - - The fixed point index for local condensing maps, Ann. Mat. Pura Appl. (4) 89 (1971), 217-258. MR 47 \#903.

96. - - The ball intersection property for Banach spaces, Notices Amer. Math. Soc. 17 (1970), 1073.

97. T. O'Neil and J. W. Thomas, On the equivalence of multiplicity and the generalized topological degree, Trans. Amer. Math. Soc. 167 (1972), 333-345. MR 45 \#7555.

98. D. Pascali, Operatori nelineari, Acad. Rep. Soc. Romania, Bucarest, 1964.

99. G. I. Petrov, Application of the Galerkin method to the stability problem of viscous fluid, Appl. Math. Mech. 3 (1940).

100. W. Petry, Existence theorems for a class of nonlinear operator equations, J. Math. Anal. Appl. 43 (1973), 250-260.

101. - - Iterative Lösung gewisser nichtlinearer Operator-gleichungen mit Anwendung auf quasilineare Differentialgleichungen, Aequationes Math. 8 (1972), 113-135. MR 46 \#6585.

102. W. V. Petryshyn, Direct and iterative methods for the solution of linear operator equations in Hilbert space, Trans. Amer. Math. Soc. 105 (1962), 136-175. MR 26 \#3180.

103. - Constructional proof of Lax-Milgram lemma and its application to non-K-p.d. abstract and differential operator equations, J. Soc. Indust. Appl. Math. Ser. B. Numer. Anal. 2 (1965), 404-420. MR 33 \#1764.

104. - On a class of K-p.d. ad non-K-p.d. operators and operator equations, J. Math. Anal. Appl. 10 (1965), 1-24. MR 35 \#7141.

105. - On the extension and the solution of nonlinear operator equations, Illinois J. Math. 10 (1966), 255-274. MR 34 \#8242.

106. - Further remarks on nonlinear P-compact operators in Banach spaces, Proc. Nat. Acad. Sci. U.S.A. 55 (1966), 684-687; see also: J. Math. Anal. Appl. 16 (1966), 243-253. MR 33 \#3148; \#6458.

107. - On a fixed point theorem for nonlinear P-compact operators in Banach space, Bull. Amer. Math. Soc. 72 (1966), 329-334. MR 33 \#1768.

108. - On nonlinear P-compact operators in Banach space with applications to constructive fixed-point theorems, J. Math. Anal. Appl. 15 (1966), 228-242. MR 34 \#1890. 
109. - Projection methods in nonlinear numerical functional analysis, J. Math. Mech. 17 (1967), 353-372. MR 36 \#2025.

110. - Remarks on the approximation-solvability of nonlinear functional equations, Arch. Rational Mech. Anal. 26 (1967), 43-49. MR 36 \#3186.

111. - Iterative construction of fixèd points of contractive type mappings in Banach spaces, Numerical Analysis of Partial Differential Equations (C.I.M.E. $2^{\circ}$ Ciclo, Ispra, 1967), Edizioni Cremonese, Rome, 1968, pp. 307-339. MR 40 \#3674.

112. - On the approximation-solvability of nonlinear equations, Math. Ann. 177 (1968), 156-164. MR 37 \#2048.

113. - On projectional-solvability and the Fredholm alternative for equations involving linear A-proper operators, Arch. Rational Mech. Anal. 30 (1968), 270-284. MR 37 \#6776.

114. - Fixed-point theorems involving P-compact, semicontractive, and accretive operators not defined on all of a Banach space, J. Math. Anal. Appl. 23 (1968), 336-354. MR 38 \#588.

115. - Nonlinear equations involving noncompact operators, Proc. Sympos. Pure Math., vol. 18, part 1, Amer. Math. Soc., Providence, R.I., 1970, pp. 206-233. MR 42 \#6670.

116. - Some examples concerning the distinctive features of bounded linear A-proper mappings and Fredholm mappings, Arch. Rational Mech. Anal. 33 (1969), 331-338. MR 39 \#3331.

117. - Invariance of domain theorem for locally A-proper mappings and its implications, J. Functional Analysis 5 (1970), 137-159. MR 42 \#914.

118. - On existence theorems for nonlinear equations involving noncompact mappings, Proc. Nat. Acad. Sci. U.S.A. 67 (1970), 326-330. MR 42 \#3631.

119. - Antipodes theorem for A-proper mappings and its applications to mappings of the modified type $(S)$ or $(S)_{+}$and to mappings with the pm property, J. Functional Analysis 7 (1971), 165-211.

120. - Surjectivity theorems for odd maps of A-proper type, Math. Ann. 192 (1971), 155-172. MR 45 \#999.

121. - On nonlinear equations involving pseudo-A-proper mappings and their uniform limits with applications, J. Math. Anal. Appl. 38 (1972), 672-720.

122. - Fixed point theorems for various classes of 1-set contractive and 1-ballcontractive mappings in Banach spaces, Trans. Amer. Math. Soc. 182 (1973), 323-352.

123. - Fredholm alternative for nonlinear A-proper mappings with applications to nonlinear elliptic boundary value problems, J. Functional Analysis (to appear).

124. - Stability theory for linear A-proper mappings, Proc. Math.-Phys. Sec. Sevčnko Sci. Soc., 1973.

125. - On the relationship of A-properness to mappings of monotone type (in preparation).

126. - , Fredholm alternative for nonlinear $k$-ball-contractive mappings with applications, J. Differential Equations (to appear).

127. W. V. Petryshyn and P. M. Fitzpatrick, On 1-set and 1-ball contractions with applications to perturbation problems for non-linear bijective maps and linear Fredholm maps, Boll. Un. Mat. Ital. (4) 7 (1973), 102-124.

128. - A degree theory, fixed point theorems, and mappings theorems for multivalued and noncompact mappings, Trans. Amer. Math. Soc. 194 (1974), 1-25.

129. W. V. Petryshyn and T. S. Tucker, On the functional equations involving nonlinear generalized P-compact operators, Trans. Amer. Math. Soc. 135 (1969), 343-373. MR 40 \#804. 
130. S. I. Pohožaev, The solvability of nonlinear equations with odd operators, Funkcional. Anal. i Priložen. 1 (1967), no. 3, 66-73. (Russian) MR 36 \#4396.

131. N. I. Pol'ski1, Projective methods in applied mathematics, Dokl. Akad. Nauk SSSR 143 (1962), 787-790=Soviet Math. Dokl. 3 (1962), 488-491. MR 26 \#3173.

132. - On the convergence of certain approximate methods of analysis, Ukrain. Mat. Ž. 7 (1955), 56-70. (Russian) MR 17, 64.

133. A. J. B. Potter, Non-linear A-proper mappings of the analytic type, Canad. J. Math. 25 (1973), 468-474. MR 47 \#5666.

134. R. T. Rockafellar, Local boundedness of nonlinear, monotone operators, Michigan Math. J. 16 (1969), 397-407. MR 40 \#6229.

135. E. Rothe, Zur Theorie der topologischen Ordnung und der Vektorfelder in Banachschen Räumen, Compositio Mat. 5 (1937), 177-197.

136. B. N. Sadovskil, Ultimately compact and condensing mappings, Uspehi Mat. Nauk 27 (1972), 81-146. (Russian).

137. J. Schauder, Invarianz des Gebietes in Funktionalräumen, Studia Math. 1 (1929), 123-139.

138. —- Der Fixpunktsatz in Funktionalräumen, Studia Math. 2 (1930), 171-180.

139. J. T. Schwartz, Compact analytic mappings of B-spaces and a theorem of Jane Cronin, Comm. Pure Appl. Math. 16 (1963), 253-260. MR 29 \#481.

140. M. Shinbrot, A fixed point theorem, and some applications, Arch. Rational Mech. Anal. 19 (1964), 255-271. MR 29 \#6323.

141. V. M. Salov, Minimum principle of a quadratic functional for a hyperbolic equation, Differencial'nye Uravnenija 1 (1965), 1338-1365. (Russian) MR 32 \#4405.

142. I. V. Skripnik, Nonlinear elliptic equations of higher order, "Naukova Dumka", Kiev, 1973.

143. P. E. Sobolevskiĭ, On equations with operators forming an acute angle, Dokl. Akad. Nauk SSSR 116 (1957), 754-757. MR 20 \#4194.

144. C. A. Stuart, The fixed point index of a differentiable $(\beta) k$-set contraction, J. London Math. Soc. (2) 5 (1972), 691-696. MR 47 \#907.

145. F. Stummel, Approximation methods in analysis, Lecture Notes Series, no. 35, Aarhus Universitet, 1973.

146. T. S. Tucker, Leray-Schauder theorem for P-compact operators and its consequences, J. Math. Anal. Appl. 23 (1968), 355-364. MR 37 \#6805.

147. M. M. Vaĭnberg, Variational methods for the study of non-linear operators, GITTL, Moscow, 1956; English transl., Holden-Day, San Francisco, Calif., 1964. MR 19, 567; 31 \#638.

148. G. Vainnikko, On the convergence of the collocation method for nonlinear differential equations, Ž. Vyčisl. Mat. i Mat. Fiz. 6 (1966), no. 1, 35-42. (Russian) MR 33 \#5129.

149. - A perturbed Galerkin method and the general theory of approximate methods for nonlinear equations, Ż. Vyčisl. Mat. i Mat. Fiz. 7 (1967), 723-751. (Russian) MR 36 \#1095.

150. - Necessary and sufficient conditions for stability of the Galerkin-Petrov method, Tartu Riikl. Ull. Toimetised Vih. 177 (1965), 141-147. (Russian) MR 36 \#1093.

151. G. N. Vaĭnikko and B. N. Sadovskiil, The rotation of condensing vector fields, Problemy Mat. Anal. Slož. Sistem Vyp. 2 (1968), 84-88. (Russian) MR 45 \#2546.

152. R.S. Varga, Functional analysis and approximation theory in numerical analysis, Conference Board of the Math. Sci. Regional Conf. Ser. in Appl. Math., no. 3, SIAM, Philadelphia, Pa., 1971. MR 46 \#9602.

153. G. Vidossich, On Peano phenomenon, Boll. Un. Mat. Ital. (4) 3 (1970), 33-42. MR 42 \#6674. 
154. J. R. L. Webb, Fixed point theorems for non-linear semicontractive operators in Banach spaces, J. London Math. Soc. (2) 1 (1969), 683-688. MR 40 \#3392.

155. - Mapping and fixed-point theorems for nonlinear operators in Banach spaces, Proc. London Math. Soc. (3) 20 (1970), 451-468. MR 42 \#917.

156. — Remarks on $k$-set contractions, Boll. Un. Mat. Ital. (4) 4 (1971), 614629. MR 45 \#2544.

157. - A fixed point theorem and applications to functional equations in Banach spaces, Boll. Un. Mat. Ital. (4) 4 (1971), 775-788.

158. H. Ship-fah Wong, Le dégrée topologique de certaines applications non-compactes, nonlinéaires, Ph.D. Dissertation, University of Montreal, 1970.

159. - The topological degree of A-proper maps, Canad. J. Math. 23 (1971), 403-412. MR 44 \#5843.

160. — A product formula for the degree of A-proper maps, J. Functional Analysis 10 (1972), 361-371.

161. S. Yamamuro, A note on d-ideals in some near-algebras, J. Austral. Math. Soc. 7 (1967), 129-134. MR 35 \#3456.

162. G. N. Jaskova and M. N. Jakovlev, Some conditions for the stability of the Petrov-Galerkin method, Trudy Mat. Inst. Steklov. 66 (1962), 182-189. (Russian) MR 27 \#5146.

163. E. Zarantonello, The closure of the numerical range contains the spectrum, Bull. Amer. Math. Soc. 70 (1964), 781-787. MR 30 \#3389. 